\title{
FERMHLAB THESIS 1986=01
}

\section{.}

s. ton

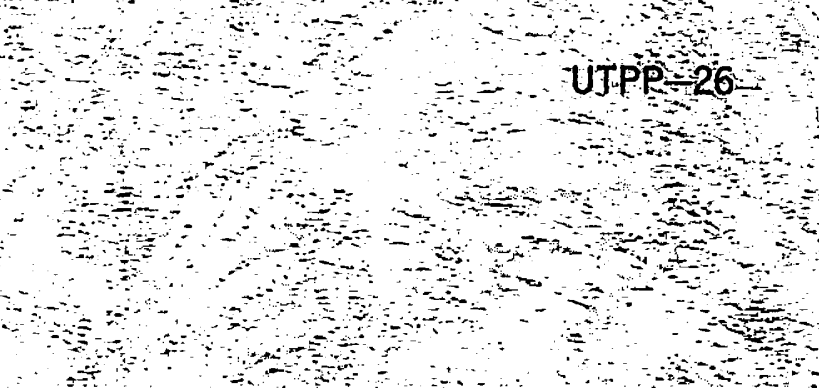

\section{CHARAOTERISTICS OF ODF ENDPUGG}

\section{ELEGTAOMAGNETCOALORIMETER}

$=-$

\section{February 1986}

\section{YOSHIO HAYASHIDE}

Sumitted inspartial fulfilmen of the requirements for

- the degree of poctor of Science

Uñiversity of Tsulkuba 
正愦表 $\frac{\text { DATE }}{\text { UंTPP-26 }} \cdot$ No.

文中 2 ×X GeV $-\Delta \Delta \Delta$
$\longrightarrow 100$$\longrightarrow\left\{\begin{array}{l}\text { electron } \\ \text { pion etc. }\end{array}\right.$

$$
\begin{aligned}
& \text { と哆ているのは }
\end{aligned}
$$

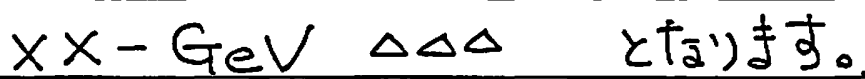

その他御气付その点は著者まで御一報下さいませ。， 
CHARACTERISTICS OF CDE ENDPLUG ELECTROMAGNETIC CALORIMETER

February 1986

Yoshio Hayashide

Submitted in partial fulfillment of the requirements

for the degree of Doctor of Science

in Doctoral program in

University of Tsukuba 

Abstract

A gas sampling calorimeter was constructed for collider Detector at Fermilab (CDF). The performance of the calorimeter was investigated with a high energy electron beam. Characteristics of the electromagnetic shower was derived from the results of the beam test. A realistic electromagnetic calorimeter simulation procedure was established as a part of $C D F$ detector simulation program. This simulation program enabled us to evaluate the detector performances in terms of capabilities for the $\vec{p} p$ collision experiment.

The electromagnetic calorimeters play an important role in recent high energy hadron collider experiments because of their excellent energy resolution for high energy electrons. They are required to have good electron identification capabilities as well as gain uniformities. A gas sampling calorimeter using conductive plastic tube with pad readout scheme allowed us to form a highly segmented calorimeter with a good gain uniformity.

The CDF endplug electromagnetic calorimeter consists of 34 layers of lead sheets and proportional chambers covering from $10^{\circ}$ to $40^{\circ}$ and from $140^{\circ}$ to $170^{\circ}$ with respect to the beam axis. Each proportional chamber consists of a planer array of proportional counters made of conductive plastic tube cathode, and pick-up electrode placed outside the array. DC field is formed between the anode wire and the conductive plastic tube, while the RE signals are picked up by the readout electrodes outside the tubes. This tube structure enabled us to form rigid proportional chambers with optimized pattern of readout electrodes.

The proportional chambers were produced following such quality control standards as flatness of the chambers and Abstract - 1 straightness of the conductive plastic tubes, to minimize their gain nonuniformity. Their stable operation was confirmed by observing anode signals induced by cosmic rays. Finally they were assembled with lead sheets at Fermilab.

The beam test of the calorimeter was undertaken at Fermilab M-bottom beam line with similar operational condition as the collision experiments at BO collision hall. The whole calorimeter was scanned by a high energy electron beam with use of a computer controlled moving stand on which the calorimeter was mounted.

The energy resolution of the calorimeter was $24 \% / \sqrt{E}$ (GeV), which was consistent with the world average. Gain saturation for $200 \mathrm{GeV}$ electrons was about $8 \%$, which is consistent with the longitudinal shower leakage and the gas gain saturation. Since the scanning procedure over all calorimeter modules took more than several days, the gas gain was traced by several gas gain monitor tubes with $\mathrm{Fe}^{55}$ radio active sources. The uniformity of the calorimeter response was better than 988 in the middle part of each module.

Event by event longitudinal shower profile obtained by the beam test was fitted to a function $f(t)=\kappa t^{\alpha} \exp (-\beta t)$, where $t$ is the depth in radiation length, to study the fluctuation of the longitudinal shower development. A strong positive correlation between $\alpha$ and $\beta$. was observed. Since the fluctuation of $\alpha$ is an order of magnitude greater than that of $\beta$, the depth of the shower maximum was determined mainly by $\alpha$. These characteristics were applied for electromagnetic calorimeter simulation program to provide realistic energy deposit in each longitudinal and lateral 
segment of the calorimeter.

The performances of the calorimeter was evaluated by simulation study as a function of the uncertainty of $2^{0}$. mass and decay width measurement. The acceptance and the calorimeter responses for the electrons from $2^{0}$ decay were studied with the detector simulation program. If we obtain an integrated luminosity of $10^{37} \mathrm{~cm}^{-2}$ and better than 18 of gain uniformity of the calorimeter responses, the mass and decay width of $z^{0}$ will be determined within $50 \mathrm{MeV}$ and $170 \mathrm{MeV}$, respectively.

The uncertainties of the Standard Model parameters determined by IVB's mass measurements at CDF will be reduced to one tenth of the current results obtained by the UA1 and UA2 collaborations. The number of additional number of neutrino families will be determined within uncertainty of $1 \sim 2$ by measuring the decay width of $z^{0}$ at the CDE.
Acknowledgments

I would like to thank Prof. K. Kondo for his guidance and encouragement throughout my graduate career.

The experiment described in this thesis has been done by collaboration of University of Tsukuba and KEK. The persons most directly involved were : Profs. K. Kondo, S. Miyashita, M. Mishina Drs. S. Kim, H. Miyata, Y. Fukui, Messrs. F. Sato, M. Ito, M. Shibata, M. Shimura, Y. Moriata, S. Kanda, Miss M. Masuzawa and the author. Thanks are due to the coworkers for their advices and collaboration in carrying out this experiment. In addition the following person made great contribution to carrying out the beam test : Dr. X. Muraki, Messrs. T. Kamon, A. Yamashita and T.Ozaki.

I wish to express my appreciation to profs. K. Takikawa, $S$. Mori, S. Mikamo, Drs. I. Nakano, Y. Takaiwa, J. Yoh, J.E. Freeman for valuable discussions.

I greatly appreciate profs. W. Carithers, W. Chinowsky, R. Ely, Drs. M.B.E.Franklin and C. Haber of Lawrence Berkeley Laboratory, University of California for making their experimental data obtained with a hadron calorimeter available to us.

The present work was carried out as a program of the Collider Detector Facility at Fermi National Accelerator Laboracory (CDF) under the accord of US-Japan collaboration in high energy physics. I wish to express my special thanks to Prof. $R$. Schwitters and Dr. A. Tollestrup (CDF leaders), Dr. K. C. Stanfield (Head of Research Devision) and Dr. L. Lederman (Director of Fermilab). 
1. Introduction 1-1

1.1 CDF detector system $1-2$

1.2 Electron detection and examples of related physics $1-3$

1.3 Calorimetry in hadron collider experiment 1-7

1.3.1 Uniform response 1-7

1.3.2 Particle identification 1-8

1.3.3 Missing Et resolution $1-9$

$1.4 \mathrm{CDF}$ Endplug Electromagnetic Calorimeter 1-10

2. Structure 2-1

2.1 Conductive plastic tubes 2-3

3. Construction and Quality Control 3-1

3.1 Production procedure of the chambers and their quality control

3.2 Cosmic ray test

3.3 Assembly

4. Experimental setup

4.1 M-bottom beam line

4.2 Readout electronics

4.3 Gas gain monitoring system

4.4 Data acquisition and online monitor

5. Results

5.1 Energy resolution

5.2 Operational voltage vs. calorimeter response

5.3 Linearity
5.6 Gain uniformity

5.7 Systematic uncertainty of the energy measurement

6. Electromagnetic calorimeter simulation

6.1 Calorimeter simulation

6.2 Parametrization of shower profile

6.2 .1 Longitudinal profile

6.2.2 Lateral profile

6.3 Comparison with beam test results

7. Discussions

7.1 Identification of $2^{0} \rightarrow \mathrm{e}^{+} \mathrm{e}^{-}$signals

7.2 Response of CDF calorimeter system

\subsubsection{Acceptance}

7.2 .2 Shower leakages

7.2 .3 Mass resolution of $2^{0}$

7.3 Results of fitting the mass peak

7.4 Discussions

7.4.1 Mass measurements

$7.4 .22^{0}$ decay width

B. Conclusions

References

Table captions

Figure captions

Tables

Figures 


\section{Chapter 1}

Introduction

The purpose of this thesis is to describe structure and characteristics of CDF (Colliding Detector at Fermilab) Endplug Electromagnetic Calorimeter and discuss their implication for the observation of some of salient phenomena expected to be observed in the $\bar{p}$ p collisions.

The recent results from CERN Spps collider experiments have demonstrated many exciting physics in the proton-antiproton collision at the center of mass energy of $540-630 \mathrm{GeV}$ region. Fermilab Tevatron I project acheived the world's highest center of mass energy, $1.6 \mathrm{TeV}$, in 1985. As shown in Fig.1-1, the collisions were observed by a subset of CDF central detector system [Ref.1-1]. Productive data taking will begin in 1986 with a full detector system.

One of the central subjects for these hadron collider experiments is to search for new heavy particles and study of their properties. The decay products of the heavy particles may be leptons, hadron jets or their combinations. In case of the hadron collider, detection of heavy particles through pure hadronic decay is generally thought to be difficult due to copious production of QCD jets. Thus the detection of leptons provides a unique clue for the identification of the heavy particles. In particular, the electron signal is quite attractive because the electromagnetic calorimeters generally give better energy resolution than the muon detectors do at higher energy.

In this Chapter, the CDF detector system is described and those physics phenomena which are identified with lepton signals in the hadron collider experiment are reviewed. The characteristics of the electromagnetic calorimeter are discussed in terms of the energy resolution, spatial resolution as well as particle identifications. Finally, the requirements from physics point of view for the electromagnetic calorimetry of CDF Endplug region and the qualification of the CDF Endplug Electromagnetic Calorimeter are described.

\section{$1.1 \mathrm{CDF}$ detector system}

CDF detector system [Ref.1-2] is divided into three parts, i.e. a central detector and two forward/backward detectors, as shown in Fig.1-2. All of them are centered on Tevatron beam line at Bo collision hall when the machine is running. Acceptance for the full calorimetry and tracking of CDF was chosen to be $-4<\eta<$ 4 in pseudorapidity which is defined by $\eta=\ln (\tan \theta / 2)$ and $0<\phi<$ $2 \pi$. The calorimeter system is segmented into projective towers to measure the energy flow into a definite region. The angular coverage of each tower is close to $\Delta \eta=0.1$ and $\Delta \varphi=5^{\circ}$ or $15^{\circ}$. Exact size of the tower depends on the detailed structure of each calorimeter.

A vertical selection through the central detector is shown in Fig.1-3. The beam pipe is surrounded by Vertex Time Projection Chamber (VTPC) which has good $\phi-z$ tracking capability. Surrounding the VTPC is a large cylindrical drift chamber which provides the precision momentum measurement in the region of $20^{\circ} \leq \theta \leq 160^{\circ}$. These two tracking detectors were located inside a thin $3 \mathrm{~m} \varnothing \times 5 \mathrm{~m}$ long, 1.5 T supper conducting solenoid magnet [Ref.1-3]. This magnet and the central tracking chamber are used to measure individual $1-2$ 
particles with $P_{t}$ less than $40 \mathrm{GeV}$.

Central calorimeter system outside the superconducting solenoid spans polar angular region between $33^{\circ}$ and $147^{\circ}$. It is composed of 48 detector unit called wedge [Ref.1-4], (Fig.1-4), each of which has a lead-scintillator type electromagnetic calorimeter and an iron-scintillator type hadron calorimeter. A wedge module has a proportional strip chamber at a depth of 5.6 radiation length (r.l.) to obtain position information of the shower. Central muon chambers surround the central calorimeter system.

The endplug electromagnetic calorimeter made of proportional chambers with cathode pad readout and lead sheets cover $10^{\circ}$ to $33^{\circ}$ and $147^{\circ}$ to $170^{\circ}$. Their detailed structure is described in the next chapter. The endplug hadron calorimeter [Ref.1-5] covering $10^{\circ}$ to $30^{\circ}$ and $150^{\circ}$ to $170^{\circ}$ has quite similar structure as the electromagnetic one.

Figure 1-5 shows an elevation view of a forward/backward region. The forward/backward detector system has gas sampling electromagnetic and hadron calorimeters with pad readout [Ref.1-6]. Muon detectors consisting of troid magnets made of magnetized iron and drift chambers to measure the muon momenta are located behind the forward/backward calorimeter systems.

\subsection{Electron detection and examples of related physics}

The CERN $\mathrm{s} \bar{p} \mathrm{p}$ collider has made it possible to produce particles with masses up to $100 \mathrm{GeV} / \mathrm{c}^{2}$ and high pt jets with invariant masses greater than $200 \mathrm{GeV} / \mathrm{c}^{2}$. As an example of physics related with the electromagnetic calorimetry, the detection of Intermediate vector Bosons (IVB's) is reviewed in this section.
Charged and neutral IVB's were discovered by CERN Sp̄pS collider through leptonic decay channels.

$$
\begin{array}{lr}
\overline{\mathrm{p} p} \rightarrow \mathrm{w}^{ \pm} \rightarrow \mathrm{e}^{ \pm} \mathrm{v}, & \text { [Ref.1-7] } \\
\overline{\mathrm{p} p} \rightarrow \mathrm{w}^{ \pm} \rightarrow \mu^{ \pm} \mathrm{v}, & \text { [Ref.1-8] } \\
\overline{\mathrm{p} p} \rightarrow \mathrm{z}^{0} \rightarrow \mathrm{e}^{+} \mathrm{e}^{-,} & \text {[Ref.1-9] } \\
\overline{\mathrm{p} p} \rightarrow \mathrm{z}^{0} \rightarrow \mu^{+} \mu^{-} & \text {[Ref.1-10] }
\end{array}
$$

The $\mathrm{w}^{ \pm \prime}$ 's were tagged by a high $\mathrm{P}_{\mathrm{t}}$ lepton and a large missing Et. The $\mathrm{z}^{0} \mathrm{~s}$ were identified by two simultaneous high pt leptons. Their masses, decay widths, Pt distributions and longitudinal momentum distributions have been reported in Ref.1-11. The number of IVB's discovered and their characteristics are summarized in Table 1-1.

Measurements for masses of $w^{ \pm}$and $z^{0}\left(m_{W}\right.$ and $\left.m_{z}\right)$ are important to test the Standard Model [Ref.1-12]. The Standard Model predictions for the $w^{ \pm}$and $z^{0}$ masses are given by :

$$
\begin{aligned}
& \mathrm{m}_{\mathrm{W}}=\mathrm{A} / \sin \theta_{\mathrm{W}^{\prime}} \\
& \mathrm{m}_{\mathrm{Z}}=2 \mathrm{~A} / \sin 2 \theta_{\mathrm{W}^{\prime}} \\
& \mathrm{m}_{\mathrm{Z}}=\mathrm{m}_{\mathrm{W}} / \cos \theta_{\mathrm{W}^{\prime}} \\
& \mathrm{A}=\left[\frac{\pi \alpha}{\sqrt{2} \mathrm{G}_{\mu}}\right]^{1 / 2}\left[\frac{1}{1-\Delta \mathrm{r}}\right]^{1 / 2}=\frac{37.281 \mathrm{GeV}}{\sqrt{1-\Delta \mathrm{r}}} \\
& \alpha=1 / 1-\mathrm{N}) \\
& \left.\mathrm{G}_{\mu}=1.16632 \times 1-\mathrm{b}\right) \\
&
\end{aligned}
$$


where $\theta_{\mathrm{W}}$ is the weak mixing angle and $\Delta \mathrm{r}$ denotes the $O(\alpha)$ radiative corrections to make eq.(1-2) is exact (Ref.1-13].

The mass difference of $z$ and $w$ is written by eliminating the weak mixing angle from eq. $(1-1)$ as:

$$
m_{Z}-m_{W}=m_{z}\left[1-\left[\frac{1+\left(1-4 A^{2} / m_{z}^{2}\right)^{1 / 2}}{2}\right]^{1 / 2}\right]
$$

Eliminating $\theta_{W}$ from Eqs.(1-1), one can find the radiative correction factor as a function of $\mathrm{m}_{\mathrm{W}}$ and $\mathrm{m}_{\mathrm{Z}}$ :

$$
\Delta r=1-\frac{(37.281 \mathrm{GeV})^{2}}{\mathrm{~m}_{\mathrm{W}}^{2}\left(1-\mathrm{m}_{\mathrm{W}}^{2} / \mathrm{m}_{\mathrm{Z}}^{2}\right)}
$$

\section{A theoretical evaluation leads to}

$$
\Delta r=0.0696 \pm 0.0020
$$

for $\sin ^{2} \theta_{W}=0.217$, Higgs mass $m_{\phi}=m_{Z}$ and top quark mass $m_{t}=36$

GeV [Ref.1-14]. Equations $(1-1),(1-2)$ and $\sin ^{2} \theta_{W}=0.217$ lead to

$$
\begin{array}{ll}
\mathrm{m}_{\mathrm{W}}=83.0+2.9-2.7 \mathrm{GeV} & \ldots(1-7-\mathrm{a}) \\
\mathrm{m}_{\mathrm{Z}}=93.8+2.4-2.2 \mathrm{GeV} . & \ldots(1-7-b)
\end{array}
$$

The mass difference between $m_{2}$ and $m_{W}$ is derived from eq. $(1-4)$ as:

$$
\mathrm{m}_{\mathrm{Z}}-\mathrm{m}_{\mathrm{W}}=10.8 \pm 0.5 \mathrm{GeV} \text {. }
$$

The smaller uncertainty of the mass difference than that of each

of the masses indicates the interdependence of $m_{W}$ and $m_{Z}$. The implications of eq. (1-4) is illustrated in Fig. 1-6 where the mass difference is plotted as a function of $m_{z}$. The mass difference decreases with increasing $\mathrm{m}_{\mathrm{Z}}$.

The weak mixing angle $\sin ^{2} \theta_{W}$ can be determined by both $m_{W}$ and $m_{z}$ as :

$$
\sin ^{2} \theta_{W}=1-\left(m_{W} / m_{Z}\right)^{2}
$$

By using eq.(1-1), it is determined by either $m_{w}$ or $m_{z}$ alone.

The radiative correction factor $\Delta r$ is separated into three parts as in eq. $(1-10)$ : the contributions of leptons and hadrons to the self-energies (Fig. 1-7), and the rest which is called the bosonic component and includes all the diagrams involving weakly interacting bosons and ghosts.

$$
\Delta r=\Delta r \text { (bosonic) }+\Delta r \text { (lepton) }+\Delta r \text { (hadron) } \ldots(1-10)
$$

The bosonic component $\Delta r$ (bosonic) depending on $m_{\phi}$ is estimated to be 0.003 with $m_{\phi}=m_{z}$. For $m_{\phi} / m_{z}<10$ suggested by considerations of perturbative unitarity (Ref.1-15], the effects of the Higgs mass are small, namely, an increase less than 0.009 in $\Delta r$.

An existence of heavy fermions lead to large negative contribution to the $\Delta r$. For example, the behaviors of $\Delta r, A$, and the predicted value of $m_{2}-m_{W}$ against $m_{t}$ are illustrated in Table $1-2$

Summarizing above, smaller $\Delta r$ or the mass difference than the prediction indicates that $m_{t}$ is significantly beyond $36 \mathrm{GeV}$. 
If those parameters are more than the predictions, the theory may need a change at the tree level.

The decay width of $z^{0}, \Gamma\left(z^{0} \rightarrow a l 1\right)$, is one of the important parameters related to additional number of neutrino species. The width were calculated from taking account of $Q C D$ radiative corrections for hadronic decay modes as listed in Table 1-3 [Ref.1-16]. Measurment of the decay width $\Gamma^{\exp }\left(2^{0} \rightarrow a l 1\right)$ gives additional number of neutrino species, $\Delta \mathrm{N}_{v}$ as

$$
\begin{aligned}
& \Delta N_{v}=\left(\Gamma^{\exp }\left(z^{0} \rightarrow a 11\right)-\Gamma^{N V=3}\left(z^{0} \rightarrow a 11\right)\right) / \Gamma\left(z^{0} \rightarrow v v\right) . \\
& \ldots(1-12)
\end{aligned}
$$

Another independent approach to counting number of light neutrino species was reported in Ref.1-17, where $N_{V}$ was derived from the relation,

$$
\frac{\sigma\left(\bar{p} p \rightarrow z \rightarrow e^{+} e^{-}\right)}{\sigma\left(\bar{p} p \rightarrow W \rightarrow e^{v}\right)}=\frac{\sigma(\bar{p} p \rightarrow z)}{\sigma(\bar{p} p \rightarrow w)} \cdot \frac{B\left(z \rightarrow e^{+} e^{-}\right)}{B\left(w \rightarrow e^{v}\right)}
$$$$
\ldots(1-13)
$$

The ratio of the production cross sections $\sigma(\overline{\mathrm{p} p} \rightarrow z) / \sigma(\overline{\mathrm{p} p} \rightarrow \mathrm{w})$ was given by $Q C D$ calculation and the experimental value was used for the left-hand side. The branching ratio $B\left(z \rightarrow e^{+} e^{-}\right)$depending on $\Delta N_{V}$ was derived from a calculation of $B(W \rightarrow e v)$. Since the precise measurement of the $\Gamma\left(2^{0} \rightarrow a l l\right)$ with small number of events is difficult, current results about $N_{v}$ are based on this calculation.
Production cross sections of IVB's calculated by LUND Monte Carlo program [Ref.1-18] with $\mathrm{K}$ factor=2 are shown in Fig.1-8. Both cross sections of $w$ and $z$ are about three times as large as those at $\sqrt{s}=540 \mathrm{GeV}$. The expected total cross section of $W$ and $z$ becomes $26 \mathrm{nb}$ and $9 \mathrm{nb}$ corresponding to 26000 and 9000 events with an integrated luminosity $\int_{L} \mathrm{dt}=10^{36} \mathrm{~cm}^{-2}\left(1 \mathrm{pb}^{-1}\right)$, respectively.

High production rate of IVB's will enables us to measure their characteristics more precisely. About 300 events of electron pair from $Z$ and more than 4000 events of ev pair from $w$ will be observed with branching ratio $B\left(Z \rightarrow e^{+} e^{-}\right)$of 38 and $B(W \rightarrow e v)$ of $8 \%$.

Events which can be interpreted as $\bar{p} p \rightarrow W \rightarrow t \bar{b}$ and $\bar{p} p \rightarrow E t$ $(t \rightarrow 1 \mathrm{Vb})$ were reported by CERN UAI group [Ref.1-19]. Twelve two-jet + high Pt lepton ( 9 electron 3 muons) events were selected from $w$ candidate events without a limitation on the jet activity. Since they are accompanied with jet activities, the electron candidates were required to be isolated and fulfill strict electron criteria.

According to their analysis, the detection probability of fraction of $Q C D$ jet satisfying the requirements is negligibly small. They concluded that the mass of the top quark, $m_{t}$ was in the range of $30<m_{t}<50 \mathrm{GeV} / \mathrm{c}^{2}$.

A possibility of such event topology coming from $\bar{p} p \rightarrow \bar{c} c X$ or $\bar{p} p \rightarrow \bar{b} b x$ were reported in Ref.1-20. Further experimental study is necessary to confirm the existence of the top quark. Assuming $25 \%$ of the branching ratio $B(W \rightarrow t \bar{b})$ and $11 \%$ of $B(t \rightarrow b e v)$, one can 
expect to observe more than $700 \mathrm{~W} \rightarrow t \bar{b} \rightarrow$ evb $\bar{b}$ events at the the Tevatron energy. Detailed study of this kinds of events will be possible in the CDF.

1.3 Calorimetry in hadron collider experiment

In this section, characteristics of a calorimeter in a hadron collider detector are.discussed considering the study of the subject described above.

1.3.1 Uniform response

As described in previous sections, precise measurements of IVB's masses and decay widths are one of important subjects for the CDE. Since the momentum of muons is determined by tracking devices with a magnetic field, the momentum resolution becomes worse at higher momentum.

On the other hand, the energy resolution of a calorimeter improves in higher energy region as a function of $1 / \sqrt{E}$. For example, IVB's masses determined by early data sample of either electrons or muons obtained by CERN UA1 and UA2 groups are compared in Table 1-4 [Ref.1-21]. The masses determined from electron samples are better than those from muons. Therefore, measurements of the electron energy is an indispensable technique for precise determination of characteristics of heavy particles.

Precise energy measurement of electrons requires good uniformity in response of a calorimeter. An intrinsic energy resolution at a certain position of the calorimeter is represented by

$$
(\sigma / E)=a / \sqrt{E}
$$$$
\cdots(1-14)
$$

where $E$ is the energy in unit of $\mathrm{GeV}$ and $a$ is an energy resolution coefficient in percent.Gev $1 / 2$. In case of an electromagnetic calorimetry, the parameter $a$ is typically about 15 $25 \%$ depending on the absorber thickness and the sampling detector. If the calorimeter has $b \&$ of gain nonuniformity, the effective energy resolution of the whole calorimeter becomes

$$
(\sigma / E)=\sqrt{(a / \sqrt{E})^{2}+b^{2}}
$$

A typical value of a leads the intrinsic energy resolution of 1-2\% for $100 \mathrm{GeV}$ scale electrons. In order to obtain good energy resolution in this energy region, the sensitivity of a calorimeter should be known to better than 1 2\%.

1.3.2 Particle identification

It is an important subject for a hadron collider detector to pick up an electron accompanied with jet activities, as is the case for $W \rightarrow$ tb events. A capability of particle identification, i.e. e/ $\pi^{ \pm}$and $e /\left(\pi^{ \pm}+n \pi^{0}\right)$ separation, is a crucial element required for electromagnetic calorimeter.

Information of energy deposits in calorimeter segments enables us to apply various kinds of cuts, e.g. ratios of energy deposits in longitudinal segments or second moments of the lateral shower spread. These procedure generally provide $10^{-2}-10^{-3}$ of pion rejection power [Ref.1-22].

A single parameter $\zeta$ which indicates an "electric likelihood" is derived from the pattern of electromagnetic shower fluctuation observed by using a highly segmented calorimeter [Ref.1-23]. If the same analysis is applied for a hadron shower, $\zeta$ takes clearly larger value than the electromagnetic one. It improves the $e / \pi$ separation by an order of magnitude.

$$
1-10
$$


A single charged track overlapping single or multiple neutral electromagnetic components such as $\gamma^{\prime} s$ from $\pi^{0}$ or $\eta^{0}$ decay may fake an electron signal. These events can be rejected from electron candidates to a large extent by requiring that the charged track points to the position of the shower on the calorimeter. Thus, the calorimeter should have good position resolution against a shower.

1.3.3 Missing Et resolution

In order to study an event accompanied with missing transverse energy (Et), such as $\mathrm{w} \rightarrow \mathrm{ev}$, the calorimeter system should have a good missing Et resolution. The calorimeter system is required to have wide coverage of pseudorapidity range and less insensitive region (crack) in between calorimeter modules. The crack can be reduced by forming the whole calorimeter system with less calorimeter modules each spanning wide coverage.

Summarizing above discussions, a calorimeter for a high energy hadron collider should have :

1. uniform response to keep good energy resolution on the whole calorimeter coverage,

2 longitudinal and lateral segments to improve particle identification,

3. large detector unit to minimize the crack.

\subsection{CDF Endplug Electromagnetic Calorimeter}

The following items are features given or required for CDF endplug electromagnetic calorimeter.

1. It is placed in magnetic field of $1.5 \mathrm{~T}$.
2. The size of each tower in this angular region $\left(10^{\circ}<\theta<40^{\circ}\right)$

$\left(140^{\circ}<\theta<170^{\circ}\right)$

is physically smaller than that in the central region $140^{\circ}<\theta$

$\left\langle 140^{\circ}\right.$, . At $\theta \sim 90^{\circ}$, the pseudorapidity range $\Delta \eta=0.1$ corresponds to $\Delta \theta=5.7^{\circ}$, while it corresponds to $\Delta \theta=1.6^{\circ}$ at $\theta \sim 15^{\circ}$. Typical size of the tower is about $5 \mathrm{~cm}$ in this angular region.

3. The electrons coming into this angular region are expected to be more energetic than those in the central region. Fig.1-9 shows the energy spectrum of electrons from $z^{0}$ decay. In the central region, the electron energy distributes around 40 GeV, while it spreads over 40 to $200 \mathrm{GeV}$ in the endplug region.

Because of the magnetic field, limited space and high radiation level around the calorimeter, the lead scintillator or the lead glass type calorimeter with phototube readolt can not be used. With these devices, it is difficult to form small unit of tower without introducing substantial cracks.

Gas sampling calorimeters with cathode pad readout system [Ref.1-24] can satisfy these requirements, because they include neither phototubes nor space consuming light guides. With this kind of gas calorimeters, any kinds of tower geometry can be formed by the cathode pad pattern. They also allow us to form fine $1-12$ 
grained segmentation in both longitudinal and lateral directions, which improves electron identification capability.

The proportional tube structure made possible to make large detector units which is necessary to eliminate the crack between the calorimeter modules. In case of lead-scintillator calorimeter, a large detector unit causes poor segmentation and bad gain uniformity because of light attenuation in the scintillators. We can obtain fine grained segmentations in the large detector unit with the cathode pad readout and the proportional tube structure. Furthermore, the construction cost of the gas calorimeter is relatively less expensive than other kinds of calorimeters.

A disadvantage of the gas sampling calorimeter is its poor energy resolution due to Landau fluctuation and path-length fluctuation as reported in Ref.1-25. In case of the CDF detector system, the energy resolution of lead-scintillator type electromagnetic calorimeter is $148 / \sqrt{E}$, while that for gas calorimeters is about $248 / \sqrt{E}$.

The poor energy resolution is not a critical issue for the calorimetry in the CDF endplug region. As an example, typical energy of an electron from $z^{0}$ decay coming into the central region is about $40 \mathrm{GeV}$, while it rises to $100 \mathrm{GeV}$ in the endplug region as shown in Fig.1-9. It should be noted that the effective energy resolutions of these two kinds of calorimeter are almost comparable considering their energy ranges. Thus, the gas sampling type calorimeter is one of the most suitable choice for CDF Endplug Electromagnetic Calorimeter.

Another short coming of the gas sampling calorimeter is a gas gain drift due to the variation of the gas pressure and the temperature. In early beam test, the gain drift of about $1 \frac{\%}{6}$ per hour was observed [Ref.4-3]. The gain drift can be traced by gas gain monitor proportional tubes with a radio active source placed in the same gas flow of the calorimeter.

In Chapter 2, the detailed structure of the CDF Endplug Electromagnetic Calorimeters is described. The construction process and the setup of the beam test are described in chapter 3 and Chapter 4 , respectively. The results of the beam test are presented in Chapter 5. An electromagnetic calorimeter simulation based on the beam test data is introduced in Chapter 6 . In Chapter

7 , the measurement of $\mathrm{z}^{0} \rightarrow \mathrm{e}^{+} \mathrm{e}^{-}$is discussed in terms of the detector performances described. 
Chapter 2

Structure of the CDF Endplug Electromagnetic Calorimeter

The two CDF Endplug Electromagnetic Calorimeters are covering from $10^{\circ}$ to $37^{\circ}$ and from $143^{\circ}$ to $170^{\circ}$ with respect to the beam axis located inside the superconducting solenoid magnet as shown in Fig.2-1. Each one forms a cylinder with a $3 \mathrm{~m}$ diameter and $50 \mathrm{~cm}$ thick. There is a $10^{\circ}$ conical hole in the middle of the cylinder.

The calorimeter consists of 34 pairs of a proportional chamber and a $2.7 \mathrm{~mm}$ thick or 0.51 radiation length ( $\mathrm{r} .1$.$) lead$ sheet. Considering the averaged incident angle of particles, the longitudinal depth becomes about 20 radiation length. Since the calorimeter is divided into four quadrants, the azimuthal angular coverage of an unit of chamber and lead sheet is $90^{\circ}$. Whole calorimeter is installed in a gas sealed vessel shown in Fig.2-2 with high voltage and signal feed through at the bottom.

The proportional chamber consists of an array of proportional counters and two sets of G-10 boards interleaving the array. The G-10 board has either pick-up electrode pattern or plane ground pattern on it. A static electric field causing a gas multiplication process is formed between an anode wire and the conductive plastic tube. Since the conductive plastic has high resistivity, more than $50 \mathrm{k} \Omega / \mathrm{sq}$, the electron avalanche signal can pass through the tube wall and induce charge on the pick-up electrode on the G-10 board placed outside the wall.

Each proportional counter consists of $0.8 \mathrm{~mm}$ thick conductive plastic tube with $7 \mathrm{~mm} \times 7 \mathrm{~mm}$ inner cross section and a $50 \mu \mathrm{m} ø$ gold plated tugsten anode wire. The wire is supported by two $Y$-shape plastic endcaps at the both tube ends. All the anode wires of a chamber are connected together to a high voltage bus line via 100 $\Omega$ resistors which prevent cross talks among them.

The G-10 boards covering one side of the proportional counter array have pads which pick up induced signal on it. The other side of the array is covered by a plane ground pattern. Chambers placed near shower maximum, at $3.27 \mathrm{r.1}$, to $8.37 \mathrm{r.1}$., have one of two kinds of strip electrode, $\theta$ stirp and $\phi$ strip, instead of the plane G-10 boards. These strip patterns shown in Fig.2-5-b, $c$ provide finer position information of a shower than pad patterns do.

The projective tower geometry of the calorimeter is defined by pads which covers $5^{\circ}$ in azimuth. The radial coverage of a pad, is $\Delta \eta=0.06$ in unit of pseudorapidity, for $1.10 \leq \eta<1.32, \Delta \eta=0.045$ for $1.32 \leq \eta<1.41$ and $\Delta \eta=0.09$ for $1.41 \leq \eta<2.40$ as shown in Fig.2-5-a. Both strip patterns span $1.2 \leq \eta \leq 1.82$ and $360^{\circ}$ in azimuth. Each $\eta$ strip covers 0.02 in pseudorapidity and $\phi$ strip covers $1^{\circ}$ in azimuth as shown in Fig.2-5-b and Fig.2-5-c, respectively.

In every $5^{\circ}$ at the outer arc of the chamber, there is a card-edge connector which is connected to the pads belonging to the 5 . sector. A tower is divided into three longitudinal segments by connecting these cardedge connectors with flat ribbon cables in order to obtain information of longitudinal shower development. Similarly, the same kind of strips are ganged into one. The longitudinal segmentation and the location of the strip pattern is listed in Table 2-1.

The mechanical support for the proportional chambers and the lead sheets is provided by 24 horizontally penetrating rods around the outer circumference of the gas vessel.
$2-2$ 


\subsection{Conductive plastic tubes}

A gas sampling calorimeter using the conductive plastic tubes has following advantages.

1. Since static electric field is formed between the anode wire and the conductive plastic tube, the shape of the pick-up cathode does not affect the field. It makes the design of the pick-up cathode easy.

2. The tube structure has an advantage in mechanical strength over plane cathode configuration, which enables one to fabricate a big proportional chamber. Making a large detector unit is essential to reduce insensitive areas in a calorimeter system.

3. In case an anode wire breaks, it will not affect other anode wires.

The conductive plastic used in the calorimeter is made by mixing carbon grains with $3 \times 10^{-8} \mathrm{~m}(30 \mu \mathrm{m})$ in diameter to polystyrene plastic. Extruding the material under proper temperature and extrusion speed, we controlled the conductivity and the mechanical accuracy of the tube wall.

The conductivity of the plastic should be high enough to obtain well localized induced signal on the pick up cathode, while too high resistivity may cause a discharge due to charge up effect on the plastic surfaces. In order to optimize the resistivity of the conductive plastic tubes, the relation between the resistivity and the spread of the signal was studied by observing the width of the distributions of induced signals on $1 \mathrm{~cm}$ wide strip readout

$$
2-3
$$

cathode for several resistive tubes with different resistivity [Ref.2-I]. This results shown in Fig.2-6 indicates that the resistivity above $30 \mathrm{k} \Omega / \mathrm{sq}$ gives practically localized $11.5 \mathrm{~cm}$ in

$\sigma)$ signal. On basis of this results, the resistivity was set to 50-100 $\mathrm{k} \Omega / \mathrm{sq}$ where the width of the distribution is almost independent of the variation of the resistivity.

The radiation damage of the tubes was tested by exposing them to doses of $10^{8} \mathrm{R}, 10^{7} \mathrm{R}$ and $10^{6} \mathrm{R}$ of $\mathrm{Co}^{60} \gamma$ rays [Ref.2-1]. No significant change of the plastic was observed in both its mechanical strength and electrical conductivity after these exposures. In comparison, the epoxy glue used to attach the wire holder was badly damaged by exposure to $10^{8} \mathrm{R}$ radiation. 
Chapter 3

Construction and Quality Control

It is important for a gas calorimeter to have proportional chambers with uniform sensitivity to eliminate the systematic uncertainty of energy measurements. There are several things which must be controlled at the production process to minimize the nonuniformity of the gas gain of the chamber. In order to establish the quality control standards for the proportional chamber production, the gain variation was measured as a function of mechanical parameters of the proportional tubes.

An experimental set-up illustrated in Fig.3-1 was used to study the relation between the wire diameter and the gain of the proportional counter. The gas gains for three anode wires with diameter of $30 \mu \mathrm{m} \sigma, 50 \mu \mathrm{m} \sigma$ and $70 \mu \mathrm{m} \sigma$ were measured by observing the responses for radio active source $\mathrm{Fe}^{55}$. The result is shown in Fig.3-2. A small gain variation, $\Delta M$ is given as a function of the small deviation of the wire diameter $\Delta R$ as

$$
|\Delta M / M|=b|\Delta R / R| \text {. }
$$

The coefficient $b$ was obtained as

$$
|\Delta M / M|=(4.8 \pm 0.5)|\Delta R / R|
$$

by fitting the data points to a function $\ln (M)=\ln (a)+b \ln (R)$ where $M$ is assumed to be written as a function of $R$ as $M=a R^{b}$.

The diameter was determined from measuring a weight and a length of a piece of wire. Samples of every wire reel were checked and those with diameter within $51.9 \pm 0.26 \mu \mathrm{m}$ which corresponded to 2.48 of gain variation were applied for the production. Wires from a single wire reel were used in a chamber to prevent local gain variation.
A displacement of an anode wire from center of the proportional tube causes the gain variation, because stronger local electric field is formed around the wire. The gain variation due to the anode wire displacement was measured by looking at the response of the anode signal for $\mathrm{Fe}^{55}$ source set on an artificially bent proportional tube [Ref.3-1]. The tube was bent in diagonal direction and parallel direction to the tube wall. As shown in Fig.3-3, the gas gain is approximately represented as a parabolic function of the displacement. A displacement less than $1 \mathrm{~mm}$ shows similar response in either direction. In this region, a fitting procedure provided the relative gas gain variation for small displacement as

$$
|\Delta M / M|=(0.214 \pm 0.006) D^{(2.33 \pm 0.06)},
$$

$$
\cdots(3-2)
$$

where $D$ is a displacement in $\mathrm{mm}$ from the center.

3.1 Production of the chambers and their quality control

The construction procedure of a proportional chamber is shown in Fig.3-4. First of all, conductive plastic tubes were arranged and glued on three pieces of G-10 sheet with pad pattern. The anode wires were stretched in the tubes. Finally, another three pieces of $G-10$ boards were glued on the tubes.

In this procedure, we paid attention to keep the proportional tubes straight to minimize the displacement of the anode wires. When the tubes were glued on the G-10 boards, they were arranged on a special jig shown in Fig.3-5 to avoid the horizontal bends of the tubes. The horizontal bends had been measured and corrected if necessary before the glue fixed. During the curing time of the glue, the chamber was pressed by a water 
tank with $50 \mathrm{~cm}$ deep water on a $3 \mathrm{~cm}$ thick iron plate to make it flat.

The flatness of a chamber and the horizontal bends of the tubes were measured for all the chambers. The averaged horizontal bend was less than $60 \mu \mathrm{m}$ and flatness variation was about $100 \mu \mathrm{m}$ in RMS. According to formula $3-2$, these values correspond to the gain change of 0.038 and $0.1 \%$, respectively.

The anode wire tension was checked in the production process in order to detect the loosen wires causing an unstable operation of a chamber. A measurement of the mechanical oscillation frequency, gives the wire tension as:

$$
T=\frac{(2 \ell v)^{2}}{\rho}
$$

$$
\ldots(3-3)
$$

where $T$ is the wire tension in Newton, $I$ is the length of the wire

in meter, $V$ is the resonance frequency in $\mathrm{Hz}$ and $\rho$ is the linear density of the wire in $\mathrm{kg} /$ meter. The wire was mechanically excited by putting it in a magnetic field and passing AC current with its resonance frequency through it.

A computer controlled wire tension checking system, schematically drawn in Fig.3-6 was designed and utilized. A pair of permanent magnet mounted on a moving table was located at the central part of the wire to which the AC current source was connected by the relay switching system. The computer scanned the frequency around expected value observing the $\mathrm{AC}$ voltage across the wire with a digital volt meter. When a bump of the voltage, a resonance peak, was detected, the wire tension was calculated. Once a chamber was set on the checking system, the computer 3- 3 automatically repeated the procedure described above. Anode wires of all the chambers were checked with this system and wires with tension less than $50 \mathrm{~g}$ were restretched with this procedure.

At the final stage of the chamber production process, high voltage loading test was applied. It was required that the dark current of the chamber was less than $1 \mu \mathrm{A}$ for $2.5 \mathrm{kV}$ in air. wiring failures or tiny fragments of the anode wires around the tube end board causing high voltage break down were detected.

\subsection{Cosmic ray test}

Anode signals induced by cosmic rays were observed for several days under similar operational condition as the final colliding beam experiment. This process, cosmic ray test, was important to confirm the stable operation of the chambers and to check whether the quality control standards at the production stage was satisfied or not.

Figure 3-7 shows the cosmic ray test system. The chambers were stacked in a gas vessel set between the upper and lower hodoscope trigger counter forming $8 \mathrm{~cm}$ by $8 \mathrm{~cm}$ trigger cells. The anode signals were amplified by LeCroy TRA1000 preamplifier and digitized by LeCroy $2285 \mathrm{ADC}$. The gas gain was monitored by a proportional tube with $\mathrm{Fe}^{55}$ radio active source during the data taking period. The chamber was operated in a gas mixture of 49.58 argon, $49.5 \%$ ethane and 18 of ethyl alcohol.

About 2000 triggers in each $8 \mathrm{~cm} \times 8 \mathrm{~cm}$ region defined by simultaneous hit of upper and lower hodoscope counter were accumulated for several days. The gain of each area on a chamber was determined by fitting the observed pulse height distribution to a Landau distribution as shown in Fig.3-8. The gain variation in a 
chamber, $\Delta G$ was defined as

$$
\Delta G=\frac{\sqrt{\frac{1}{N} \Sigma_{i}\left(G_{i}-\langle G\rangle\right)^{2}}}{\langle G\rangle} .
$$

$$
\ldots(3-4)
$$

where $G_{i}$ is the gain of $i-t h$ region, $\langle G\rangle$ is mean gain of the chamber and $\mathrm{N}$ is the number of regions in the chamber. The $\Delta G$ was from 2 to $6 \%$ as shown in Fig.3-9. This gain variation, which was bigger than expected could be attributed to the poor flatness of the bottom plate of the gas vessel, because it might make the intrinsic flatness of the chamber worse. Chambers showing systematic gain variation or unstable behavior during the cosmic ray tests were inspected and repaired.

\subsection{Assembly}

The construction of the chambers and the cosmic ray test were carried out in Japan. The chambers which passed the cosmic ray test were shipped to Fermilab to be assembled with lead sheets. They were inspected to find out mechanical damages during the transportation. Electrical checks such as continuity test of anode wires or high voltage loading test were applied.

occasionally, the connection between a pad and corresponding card-edge connector was lost because of stains on the card-edge connectors or damaged printed circuit patterns on the G-10 board. Since these failures affect the gain uniformity of the tower signals, they were repaired before assembling them with the lead sheets.

In order to detect such failures, an automatic pad connection checking system shown in Fig.3-10 was utilized for all pads and strips of the calorimeter. It pulsed anode wires via the high voltage connector, and read the signals induced on the pad surface via the card-edge connector with LeCroy 2285 ADC. Although the pulse height of the pad signal depended on their capacitance, signals of disconnected pads were clearly distinguishable as shown in Fig.3-11. Thus the computer was able to make a diagnosis of the connection.

The lead sheets were precisely machined to fit the corresponding chambers. Their thicknesses were measured by an ultrasonic thickness gauge. According to the measurement, a common pattern of the thickness as shown in Fig.3-12 were observed. The variation was about $30 \mu \mathrm{m}$ in RMS, which is not effective for the gain variation. In order to prevent electrical contacts between the lead sheet and the printed circuit pattern on the chamber, 50 $\mu \mathrm{m}$ thick G-10 sheets were glued on the surface of the lead sheets.

Since the chambers and the lead sheets are supported by 24 rods around the outer circumference of the gas vessel, they must be rigid enough to keep the flatness by themselves. In order to satisfy this requirements, four or five pairs of a chamber and a lead sheet were glued together to make them rigid.

After making blocks of glued chambers and lead sheets, they were installed in a gas sealed vessel. The card-edge connectors of the chamber were connected together by flat ribbon cables which were fed to the signal through connectors at the bottom of the gas vessel. Connections between the pad and the signal-through connector outside the gas vessel were confirmed by a similar procedure as described above. 
Experimental Setup

\subsection{M-bottom beamline}

The calorimeter was tested and calibrated at Fermilab M-bottom beam line where we obtained electron and hadron beams with energy range from $20 \mathrm{GeV}$ to $200 \mathrm{GeV}$. The hadron beam was generated by shooting the aluminum target with a primary $800 \mathrm{GeV}$-proton beam. The electron beam was obtained by sweeping out the secondary charged hadrons with a magnet and converting residual $\gamma^{\prime}$ s originated from $\pi^{0}$ 's into $\mathrm{e}^{+} \mathrm{e}^{-}$pairs.

A couple of dipole magnet and four sets of beam PWC's were arranged on the beam line to tag the position and the momentum of each incident particle as shown in Fig.4-1. Horizontal and vertical resolutions of the PWC system were $0.5 \mathrm{~mm}$ and $1 \mathrm{~mm}$, respectively. The momentum resolution of this system was $0.47 \%$ for $100 \mathrm{GeV}-\mathrm{elect}$ rons which is small enough compared with the energy resolution of the tested calorimeter [Ref.4-1]. The beam transportation system upstream of the tagging system determined the nominal momentum of the beam. The momentum spread was about 28 for $100 \mathrm{GeV}$ electrons as shown in Fig.4-2.

The trigger was defined by three trigger counters and a veto

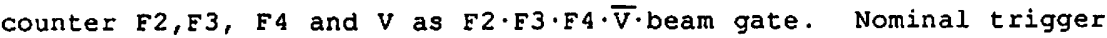
rate was about $1 \times 10^{4}$ events/burst.

The calorimeter was mounted on a computer controlled rotation stand. The stand was rotated around a pivot point on horizontal plane. The calorimeter was revolved about its centroid. This configuration simulated the incident particles coming from the vertex point of the pp collision. Every tower of the calorimeter was exposed to the test beam with this system.

Two rotary encoders with resolution of $14 \mathrm{bit} / 360^{\circ}$ had been installed at the pivot point and the central shaft of the calorimeter. A hydraulic cylinder pushed the stand horizontally and a hydraulic motor with two friction rollers were used to rotate the calorimeter around its centroid. Both the cylinder and the motor were controlled either manually or automatically. Figure 4-3 shows the control system of the rotating stand.

Each center of pad was selected as a calibration point as illustrated in Fig.4-4. The stand was rotated horizontally from one calibration point to another in every interval between two successive beam spills.

\subsection{Readout electronics}

Pad, strip and wire signals from the calorimeter were connected to frontend electronics system, called RABBIT (Redundant Analog Based Bus Information Transfer) [Ref.4-2].

The RABBIT system is essentially the same system as used in the pp colliding beam experiment at Bo collision hall. In case of colliding beam experiment, it is not easy to access the detector system including the frontend electronics during the experiment. A highly redundant frontend electronics system like RABBIT is suitable for such experiments because it can reduce the total failure rate of the system. For example, a RABBIT Hutch shown in Fig.4-5 has two sets of bus lines (TOP and BOTTOM) on its back plane. Every module on the RABBIT back plane can be accessed via either pass.

At the beam test, the RABBIT Hutch had charge sensitive 
amplifier modules called CARROT, a scanner/digitizer module called EWE and a timing generator module for sample and hold circuits of the amplifier called BAT as illustrated in Fig.4-6. The CARROT amplifier module consists of 24 channels of charge sensitive amplifiers with fast outputs for the main trigger system and their calibration circuit, as shown in Fig.4-7. Each amplifier channel has two sample and hold circuits. Since the fall time of the amplifier $C_{f} R_{f}=100 \mu s e c$. is longer than the $\bar{p} p$ beam crossing time, $2.5 \mu s e c$, the output signals of the amplifier may pile up on the previous signals. The two sample and hold circuits hold the output voltage sampled before and after the beam crossing to obtain a net pulse height. The control signal of these circuits shown in Fig.4-8 are generated by the BAT module. The EWE module reads the two signals via the analog bus lines on the back plane. It subtracts the signal sampled before the event from that sampled after the event and digitizes the difference with a 16 bit ADC.

In the $\bar{p} p$ collision experiment at BO, the $16 \mathrm{bit}$ data from EWE module are read by an intelligent read out processor called MX. In the beam test, the digitized data of all the connected channels are read by a CAMAC system via a CAMAC-RABBIT interface module instead of the $M X$. The basic function of the interface module is to transfer data from the host computer to the EWE module and vice versa. In order to read the pulse height of an amplifier channel, the host computer transfers the channel address to be accessed to the EWE module, waits for the digitizing time and reads the digitized data. This process is repeated until all channels have been scanned. There were about 500 channels to be read and more than 500 events were required per spill (about $20 \mathrm{sec}$.).

At the early stage of the beam test, the CAMAC-RABBIT $4-3$ interface module translated each CAMAC read/write instruction to that of RABBIT system. Every access to the EWE had to be accompanied with a CAMAC command. It took about $70 \mathrm{msec}$. to scan all the channels so that we were able to take less than 300 events per spill. Almost half of the time was spent by dealing with the CAMAC instructions.

The total read out time was reduced by introducing a sequencer on the interface module which controlled the read or write processes of the EWE module instead of a series of CAMAC commands issued by the host computer. The interface had $32 \mathrm{~kb}$ it memory space consisting of two $16 \mathrm{kbit}$ static random access memory chips as schematically shown in Fig.4-9. Channel addresses to be transferred to the EWE module were downloaded in the upper $16 \mathrm{kbit}$ memory space before the data taking. The data taken from the EWE were stored in the lower $16 \mathrm{kbit}$ space. Once the interface module was triggered, it repeats this process until the number of data in the memory was equal to the preset value. During this process, the interface module did not interfere with the CAMAC busline, so that the host computer worked on the other job such as reading out PwC information. The data accumulated in the memory space was read by the host computer with direct memory access method which took only $2 \mu \mathrm{sec}$. per word transfer.

Using two RABBIT Hutches and dedicated new interface modules, the dead time per event was reduced to $20 \mathrm{msec}$. We took about 1000 events per spill.

\subsection{Gas gain monitoring system}

According to the results of preliminary beam test in 1984 [Ref.4-3], the gas gain varied about $30 \%$ in two weeks and maximum 
of 18 in an hour as shown in Fig.4-10. A result of a bench test shown in Fig.4-11 indicates that the variation of the gas gain is given by a function of a pressure/temperature as

$$
|\Delta M / M|=(7.8 \pm 0.5)|\Delta(P / T) /(P / T)|,
$$$$
\ldots .(4-1)
$$

where $M$ is a gas gain, $P$ is a pressure and $T$ is a temperature in $\mathrm{K}$. Since the calibration runs took more than two weeks to scan all the towers of a plug, it was necessary to trace the gas gain as a function of time.

The same proportional counter used in the calorimeter was assembled as a gas gain monitoring tube with a $1 \mu \mathrm{Ci} \mathrm{Fe}^{55}$ line source. Twenty four such tubes for a single plug were installed in a gap between the calorimetex and the gas vessel.

Output signals of more than six tubes were observed continuously during the calibration runs. The read out system of the gas gain monitoring system is shown in Fig.4-12. The signal for the monitor tube was so small that it was fed to LeCroy TRA1000 preamplifier. One of the two outputs of the amplifier was connected to LeCroy 2285 ADC via a $105 \mathrm{nsec}$ delay line. The other output, which had opposite polarity, was fed to the discriminator via an inverter and a main amplifier to obtain enough high pulse height against the threshold of the discriminator. The threshold of the discriminator was adjusted to about half of the main peak pulse height. The trigger of the $\mathrm{Fe}^{55}$ source signal was defined by the logical OR of these discriminator outputs in the interval of successive beam spills. The trigger was prescaled to obtain about 5000 triggers at every calibration point, which corresponded to 0.148 of statistical error.
4.4 Data acquisition and online monitor

Figure 4-13 shows the entire data taking system. There were four parallel CAMAC crates and two serial CAMAC crates connected to Jorway 411 branch driver. Interrupt requests were accepted by a CAMAC interrupt controller called Bison Box. When it accepted an interrupt, it demanded the CPU to handle it, and transmitted 'CPU busy' signal to the trigger electronics to suppress next interrupt until the data processing was completed. Both the branch driver and the Bison Box were controlled by PDP-11/45 computer.

The trigger system is shown in Fig.4-14. Beside the beam trigger and source trigger mentioned before, there were two kinds of triggers called, pedestal trigger and pulser trigger. The pedestal trigger were defined by accidental hits of F2.F3 in a beam spill to read pedestals of all channels. The pulser trigger was defined by a trigger output of a pulse generator to take the calibration data of the CARROT cards and to test the cable connection between the gas vessel and the CARROT cards. Logical OR of these four triggers was transmitted to the Bison Box and the Ding-Bat, which controlled the BAT module in the RABBIT Hutch.

Commonly used online program at Fermilab, MULTI, took the data and wrote them on $6250 \mathrm{bpi}$ magnetic tape for later offline analyses. This program also provided online analysis and various monitoring capability. During the data taking process, we monitored the beam profiles, current of the tagging magnet, position of the rotating stand, momentum distributions, total pulse height of the calorimeter as well as the source signals.

Before starting the data acquisition, the connection between the gas vessel and the CARROT cards were checked by applying similar method used in construction procedure of the calorimeter. 
As shown in Fig.4-15, several anode wire signal lines belonging to every longitudinal segment were disconnected from the wire amplifier card and connected to the pulser by remote controlled relay switch. Pulsing the chambers via the high voltage network and high voltage cables, we could observe the induced signals of each tower. Incomplete connection of the cables were detected and fixed before the data taking.

\subsection{Gas system}

Mixture of $50 \%$ Argon and $50 \%$ ethane was supplied to the calorimeter through the ethyl alcohol bubbler which was kept at

$-2 \cdot \mathrm{c}$. The gas composition in the calorimeter was Argon : ethane: ethyl alcohol=49.2:49.2:1.6.

The calorimeter was operated at pressures of 1 atm with gas flow rate of $100 \sim 200 \mathrm{~cm}^{3} /$ minute.
Chapter 5

Results

5.1 Energy resolution

The energy resolution of the calorimeter was obtained by fitting the total pulse height distribution to Gausian distribution as shown in Fig.5-1. The data were taken at $\theta=26.3^{\circ}$ where the calorimeter covered from 0.8 r.1. to 20.7 r.1.. Figure 5-2 shows the energy resolution obtained at operational voltages of $1.75 \mathrm{kV}, 1.80 \mathrm{kV}, 1.85 \mathrm{kV}$ and $1.90 \mathrm{kV}$ as a function of $1 / \sqrt{\mathrm{E}}$ where $E$ is an electron beam energy in unit of GeV. The energy resolution of both the pad and the wire signals are $21-238 / \sqrt{E}$ at the operational voltage above $1.80 \mathrm{kV}$. The energy resolution corresponds to $308 \sqrt{t / E}$ where $t$ denotes the sampling thickness in unit of radiation length. It is consistent with the world average as compared in Table 5-1.

\subsection{Operational voltage vs. calorimeter response}

Figure 5-3 shows a response of the calorimeter vs. operational voltage. Above $1.90 \mathrm{kV}$, a saturation of the gain is observed for $100 \mathrm{GeV}$ and 160 Gev-electrons. Eitting the logarithmic data points below $1.90 \mathrm{kV}$ to a line, leads the gradient of the line $G$ as :

$$
\begin{aligned}
G(E=100 \mathrm{GeV}) & =9.27 \pm 0.01 / \mathrm{kV} & \ldots \ldots(5-1) \\
G(E=160 \mathrm{GeV}) & =9.20 \pm 0.01 / \mathrm{kV} . & \left.\ldots .(5-1)^{\prime}\right)
\end{aligned}
$$

This results shows that the gain change $\Delta M$ is given by a function of voltage deviation $\Delta \mathrm{V}$ as :

$$
|\Delta M / M| \sim 9|\Delta V / V| \text {. } \quad \ldots \ldots(5-1 \cdots)
$$

The operational voltage was set to $1.80 \mathrm{kV} \pm 1 \mathrm{~V}$ in which the voltage fluctuation of $1 \mathrm{~V}$ corresponds to 0.58 of the gain variation around 5- 1 


\subsection{Linearity}

The calorimeter response as a function of a beam energy is shown in Fig.5-4. Each line in this figure is determined by the lowest two data points. A gain saturation for higher energy electrons is observed at every operational voltage.

In order to evaluate the saturations more quantitatively, all the data points of the wire signal at $1.80 \mathrm{kV}$ were fitted by a polynominal of an electron energy $E$ (GeV) as

$$
f(E)=P_{1} E-P_{2} E^{2}, \quad \ldots \ldots(5-2)
$$

where $E$ was in $\mathrm{GeV}$ and $f(E)$ denoted the pulse height in unit of $A D C$ count. The data points and the fitted curve is shown in Fig.5-5. An averaged deviation of the data points from the curve is about 0.58 .

The gain saturation is evaluated as a deviation of a data points from a line whose gradient is the derivative of the polynominal (5-2) at $E=0$. In this case, the line is represented by

$$
y=P_{1} E \text {. } \quad \ldots(5-3)
$$

Thus the saturation against the expected value is represented by

$$
\begin{array}{ll}
1-f(E) / P_{1} E & \ldots \ldots(5-4) \\
=\left(P_{2} / P_{1}\right) E . & \ldots \ldots(5-4)
\end{array}
$$

According to the fitting, $\left(\mathrm{P}_{2} / \mathrm{P}_{1}\right)$ is $0.41 \times 10^{-3} \mathrm{GeV}-1$ so that the saturation for $200 \mathrm{GeV}-\mathrm{electron}$ beam becomes 8\%. Extrapolating to the the electron energy of $400 \mathrm{GeV}$, we expected gain saturation of $16 \%$. Although we assumed that the lowest two data points do not saturate at all in the previous simple linear fit, this evaluation gives 1.68 of gain saturation even for $40 \mathrm{GeV}$-electrons.

\section{3 .1 Shower leakage estimation}

A shower leakage due to limited longitudinal coverage of the calorimeter was estimated by integrating the longitudinal shower profile.

Figure 5-6 shows the averaged longitudinal shower profile at electron energy from $20 \mathrm{GeV}$ to $200 \mathrm{GeV}$. These profiles were fitted by a function

$$
y=K t^{\alpha} \exp (-\beta t),
$$

where $t$ is the position in radiation length, $k, \alpha$ and $\beta$ are functions of an incident electron energy. The incident energy dependence of $\alpha$ and $\beta$ are shown in Eig.5-7. The parameter $\alpha$ and $\beta$ are fitted by a function of $\ln (E)$ as :

$$
\begin{aligned}
& \alpha=1.2+0.69 \ln (E), \\
& \cdots(5-6) \\
& \beta=0.47+0.014 \ln (E) \text {. } \\
& \text {.... (5-7) }
\end{aligned}
$$

The parameter $\alpha$ shows clear In(E) dependence, while $\beta$ is almost constant value 0.53 in this energy range.

The energy deposit in an infinitely deep calorimeter is estimated by integrating the shower profile along the depth $t$ from 0 to infinity. Similarly, the energy deposit in an actual calorimeter is obtained by integrating the profile over its active longitudinal coverage depending on the incident angle of the beam. Fraction of the shower leakage and the invisible component absorbed in a front plate of the calorimeter is represented by 


$$
1-\frac{\int_{t}^{b} t^{\alpha} \exp \left(-\beta_{t}\right) d t}{\int_{0}^{\infty} t^{\alpha} \exp \left(-\beta_{t}\right) d t}
$$

where $a$ and $b$ are $0.80 \mathrm{r.1}$. and $20.7 \mathrm{r.1.}$, respectively. As shown in Fig.5-8, the result indicates 3.58 of shower leakage for $200 \mathrm{GeV}$-electron beam. Even for $20 \mathrm{GeV}$-electron beam, 18 of leakage is observed, which causes the nonlinearity in the low energy region.

Figure 5-9 shows the beam angle dependence of the shower leakage. Because of the limited diameter of the calorimeter, the calorimeter does not have enough longitudinal coverage for polar angle more than $30^{\circ}$. It causes steep rise of the leakage at the outer circumference. In the inner region, the leakage changes from 28 to 48 for $100 \mathrm{GeV}$-electrons.

A contribution of the shower leakages to the energy resolution was studied by removing signals from chambers placed at the back of the calorimeter. Figure 5-10 shows the results. For 40-GeV electrons, the resolution with 26 layers is almost same as that with 34 full layers. Although the leakage for the 200 GeV-electrons becomes 3.5\%, the energy resolution does not change with more than 32 layers (19 r.l.).

\subsubsection{Gas gain saturation}

Output of each chamber for $160 \mathrm{GeV}$-electrons was compared at different operational voltages as shown in Fig.5-11. The horizontal axis is the charge outputs at an operational voltage of $1.70 \mathrm{kV}$ and vertical axis is those at $1.80 \mathrm{kV}$. If the gain saturation is uniform over all chambers, these plots should be on a straight line as shown Fig.5-11-a.

If we choose $1.90 \mathrm{kV}$ as the higher operational voltage (Fig.5-11-b), the plots are not on a line. The plots located at the backward part of the calorimeter are on a line with steep gradient, where the charge density is not as high as those near shower maximum. This observation suggests that the charge density contributes to the the gain saturation.

\subsection{Lateral informations}

Averaged energy deposit among the towers for $100 \mathrm{GeV}$ is shown in Fig.5-12. A tower exposed to the bearn contains about from 408 to 708 of total energy deposit depending on the radial location of the tower.

The transverse spread of the shower was evaluated by a least square fit. In this analysis, we picked up events passing through $1 \mathrm{~cm} \times 1 \mathrm{~cm}$ area at PWC2 and $2 \mathrm{~mm} \times 2 \mathrm{~mm}$ area at PWC3 or PWC4. Since the distance between PWC2 and PWC 3 was $26 \mathrm{~m}$ and that between PWC 3 and the calorimeter was about $3 m$, the beam spread on the calorimeter was $3 \mathrm{~mm} \times 3 \mathrm{~mm}$. It was assumed that a deposit in a tower was given by integrating a density function over the pad area. The function was specified by a coordinate of the shower centroid on the calorimeter and a shower spread parameter.

Parameters which minimizes the following $\chi^{2}$ :

$$
\chi^{2}=\sum_{i}\left[\frac{p_{i}-\int_{\phi_{i}}^{\phi_{i+1} \int_{r_{i}}^{r_{i+1}} f\left(r^{\prime}\right) r d r d \phi}}{\sigma_{i}}\right]^{2}
$$

was searched. 
The density function $f\left(r^{\prime}\right)$ was given by

$$
f\left(r^{\prime}\right)=A \exp \left(-x^{\prime} / R\right) \text {, }
$$

where the free parameter in this fitting procedure were $r_{S}, \phi_{S}$ and R.

Parameters included in eq. (5-9) were defined as :

$p_{i}$ : averaged pulse height at the $i-t h$ tower,

$\sigma_{i}:$ RMS of the pulse height at the i-th tower,

$r_{i}, r_{i+1}, \phi_{i}$ and $\phi_{i+1}$ : boundaries of the $i-t h$ tower

$\mathbf{r}_{\mathbf{s}}$ : coordinate of center of shower specified by $r_{s}$ and $\phi_{S}$,

$r^{\prime}=\left|x-r_{s}\right|=\sqrt{r^{2}+r_{s}^{2}-2 r r_{s} \cos \left(\phi-\phi_{s}\right)}$.

The observed energy deposit and the simulated one with $\mathrm{R}=1.7 \mathrm{~cm}$ were quite similar as compared in Fig.5-13. The spread parameter $R$ was almost independent of the incident energy as shown in Fig. 5-14.

The same analysis was applied for the strip signals. In this case, the $R$ was about $1.6 \mathrm{~cm}$ for both $k$ inds of strip. The parametrization agreed with the observed data as shown in Fig.5-15. Position resolutions of pad and strip signals were studied by calculating the distance between hit position of the beam PWC and the centroid of the shower. The shower centroid was determined for each event by

$$
\langle x\rangle=\Sigma_{i} P_{i} x_{i} / \Sigma_{i} P_{i}, \quad \ldots \ldots(5-11)
$$

where $P_{i}$ was a pulse height observed at the i-th pad or strip and 5- 6 $x_{i}$ was a centroid of them in either $\theta$ or $\phi$ direction.

Energy dependence of the resolution is shown in Fig.5-16. Using the strip information, we can determine the center of the shower with an accuracy of $1.5 \mathrm{~mm}$ in $\sigma$ at the shower maximum. The second pad segments show $2-3 \mathrm{~mm}$ of position resolution. The resolutions of the first and the third pad segment are not as good as those observed at the second pad segment or strip because of less energy deposits in those segments as shown in Fig.5-17.

5.5 Gas gain monitor

Pulse height distribution of $\mathrm{Fe}^{55}$ source observed by the monitor tubes are shown in Fig.5-18. Since all the channels were read out synchronizing a trigger defined by logical OR of the signal from all the tubes, a pedestal value of a channel was taken with the trigger hit by the other tubes. Both the signal and the pedestal distributions were fitted to a Gaussian distribution.

Some distributions were distorted by noise as shown in Fig.5-18-b. Fitted results of these distribution were rejected by looking at a reduced $\chi 2$ and $\sigma / \mu$ of the fit. In case of successful fit as shown in Figure $5-18-a$, the $\chi 2$ was $\sim 1$ and $\sigma / \mu$ distributed around 0.1 . Figure 5-19. shows the scatter plot of the reduced $\chi^{2}$ vs. $\sigma / \mu$. Plots satisfying $\chi^{2} \leq 3$ and $0.05 \leq \sigma / \mu \leq 0.12$ (shadowed area in Fig.5-19) was selected as an acceptable data.

Gas gain at each calibration point obtained by weighted mean value of the working tubes was traced smoothly as shown in Fig.5-20. Gas gain of all runs are obtained as shown in Fig.5-21

$$
\text { 5- } 7
$$


by averaging the data points within a run.

\subsection{Uniformity}

A response map of the wire signal was obtained as shown in Fig.5-22 by scanning all the calorimeter. All quadrants shows regular response pattern.

The gain uniformity of each quadrant was about $98 \%$ in RMS as shown in Fig. 5-23 within a central region defined by $1.26 \leq \eta \leq 2.22$ and $10^{\circ} \leq \phi \leq 80^{\circ}$ of each quadrant. Figure 5-24-a and 5-24-b show the responses along the $\phi$ and radial direction, respectively. Even in the central region, the response fell down systematically at small $\theta$. It was attributed to an incident angle dependence of the longitudinal shower leakage.

The uniformity was improved better than 28 in RMS by correcting all the data points with the expected leakages as shown in Fig.5-9-a (Fig.5-25). The systematic fall down disappeared as shown in Fig. 5-26.

Responses of a peripheral part of a quadrant are shown in

Fig.5-27. At $\eta=1.15 \quad(\theta=35.1 \%)$, the responses were about 20-408 of those in the central region, which was consistent with the leakage estimation described in 5.3.1.. About 20-308 of total energy leaked into the $10^{\circ}$ cone at $\eta=2.35$.

There was an insensitive area due to 1-2 $\mathrm{mm}$ wide physical gap between quadrants and $3 \mathrm{~mm}$ thick plastic endcap holding the anode wires. Although the energy deposit was evaluated by a sum of both quadrants at scanning points adjacent to the crack between quadrants, the deposit varied 50-95\% of those in the central area since each scanning point was placed on a center of pad, the distance between the scanning point and the crack became closer at smallex $\theta$.

5.7 Systematic uncertainty of the energy measurement Uncertainty of the absolute energy measurement can be affected by following factors:

1. ambiguity of the operational voltage,

.2. absolute momentum of the test beam,

3. the absolute gain of the readout electronics,

4. uncertainty of the gas gain monitor.

The first item depends on the accuracy of regulating the high voltage power supply. We set the operational voltage with accuracy of four digits which corresponded to $0.5 \%$ of the gas gain uncertainty according to eq. (5-3).

The absolute momentum of the test beam was determined by the current of the tagging magnet. It was monitored with accuracy of three digits so that the uncertainty was 0.28 for 100 GeV-electrons.

As mentioned in Chapter 4, every amplifier card has its own calibration pulse circuit which injects charge into individual amplifier channel. The accuracy of the injected charge depends on the the charge-up. capacitor, which is known to better than 18 .

The ambiguity of the gas gain monitored by $\mathrm{Fe}^{55}$ source is characterized by the statistical error and the systematic error mainly comes from the calibration of the electronics. Number of source signals we took at each calibration point corresponded to 0.148 of the statistical error. The systematic error was estimated to be 1.58 
The first and the second effects are not major source of the systematic uncertainty of the energy measurement. The third and the fourth effects lead the systematic uncertainty of $1.8 \%$.
Chapter 6

Electromagnetic Calorimeter simulation

\subsection{Calorimeter simulation}

A detector simulation program provides responses of a detector system for each particle included in an event. It is necessary to understand the detector responses of aiming event and to estimate the effects of background against the signal.

The CDF detector simulation program traces the particles produced at the event vertex through the detector region until it stops by decaying, converting, showering in the calorimeter or exiting the CDF detector volumes. As the particle traverses through various detector regions, it suffers from energy loss $(\mathrm{dE} / \mathrm{dx}$ ) and multiple scattering and generates information such as wire hit of tracking chambers or energy deposition in calorimeters.

The calorimeter simulation in this program provides energy deposition in each calorimeter segment. It should satisfy the following requirements.

1. The shower profile in the longitudinal and lateral directions should provide realistic energy sharing between towers and leakages out of the calorimeter.

2. Statistical characteristics of the shower fluctuation should be reproduced to apply the $e / \pi$ separation algorithm as mentioned in Chapter 1 for the simulated data.

Because of high particle multiplicity of high energy $\bar{p} p$ collision events, time consuming shower simulation programs such as EGS [Ref.6-1] are not suitable for this purpose.

A new parametrization method of shower profile have been established by studying the beam test results of the CDF Endplug 
Electromagnetic Calorimeter. It reproduces not only averaged shower profiles but also statistical shower fluctuations.

\subsection{Parametrization of shower profile}

6.2.1 Longitudinal profile

An event by event energy deposit of an electron with energy $E$ in a longitudinal calorimeter segment is given by an integration of a shower profile :

$$
E_{i k}{ }^{\text {true }}=\int_{\Delta i} f_{k}(t) d t, \quad \ldots(6-1)
$$

where $E_{i k}{ }^{t r u e}$ is the energy deposit of the $k$-th event into the $i-t h$ segment, $\Delta i$ is the integral region (i.e. longitudinal coverage) and $f_{k}(t)$ is a longitudinal shower profile of the $k$-th event. Although the actual calorimeter in the CDF consists of alternative layers of an absorber and a sampling detector, it is treated as uniform material in the simulation program. It is assumed that the shower profile $f_{k}(t)$ fluctuates event by event keeping its integration :

$$
E=\int^{\infty} f_{k}(t) d t
$$

Since the observed energy deposit $E_{i k}$ is smeared by the energy resolution of the calorimeter, it is represented by

$$
E_{i k}=E_{i k}{ }^{\text {true }}+R r_{k} \sqrt{E_{i k}{ }^{\text {true }}}, \ldots(6-3)
$$

where $R$ is the detector dependent energy resolution factor and $r_{k}$ is a random number which follows Gaussian distribution with unit standard deviation. Since the calorimeter is assumed to be made of uniform material, the energy resolution has nothing to do with the fluctuations of the shower but it is mainly determined by the resolution factor $R$ as $\sigma / E=R / \sqrt{E}$ in case of small shower leakage.
The fluctuation of $f_{k}(t)$ causes the correlation of energy deposits among the longitudinal segments.

As the shower profile, a function

$$
\begin{aligned}
& f_{k}(t)=K t^{\alpha_{k} \exp \left(-\beta_{k} t\right)} \quad \ldots \ldots(6-4) \\
& K=E / \infty_{t} \alpha_{k} \exp \left(-\beta_{k} t\right) d t \\
& \left.\ldots \ldots(6-4)^{\prime}\right)
\end{aligned}
$$

was chosen, where $t$ is in unit of radiation length. The parameter

$\alpha_{k}$ and $\beta_{k}$ were decomposed into fixed, and fluctuating parts:

$$
\begin{aligned}
& \alpha_{k}=\langle\alpha(E)\rangle+\Delta \alpha_{k}(E) \\
& \beta_{k}=\langle\beta(E)\rangle+\Delta \beta_{k}(E) .
\end{aligned}
$$

In order to study the statistical characteristics of $\alpha_{k}$ and $\beta_{k}$, event by event anode wire signals of the CDF Endplug Electromagnetic Calorimeter were fitted to the function eq.(6-4). Examples of the event by event shower profiles induced by 100 Gev-electrons with fitted curves are shown in Fig.6-1. The distributions of $\alpha_{k}$ and $\beta_{k}$ shown in Fig.6-2 were fitted to Gaussian distributions. The energy dependence of the mean and standard deviation are shown in Fig.6-3. The mean value of $\alpha$ shows clear In (E) dependence. Since the function $(6-4)$ is an approximation of the true shower profile, the integration of the function over the segment gives slightly different mean energy deposit from the real data. These parameters were eventually modified so as to reproduce the observed mean energy deposit. The final parameters are listed in Table $6-1$. 
The $\alpha_{k}$ and $\beta_{k}$ have strong positive correlation as shown in Fig.6-4.

The correlation coefficient $c_{\alpha \beta}$ defined by

$$
c_{\alpha \beta}=\frac{\langle\alpha \beta\rangle-\langle\alpha>\beta\rangle}{\sqrt{\left(<\alpha^{2}>-\left\langle\alpha p^{2}\right)\left(<\beta^{2}\right\rangle-\langle\beta\rangle^{2}\right)}}
$$

was about 0.8 over all energy region.

The function (6-4) takes a maximum value at $t_{\max }=\alpha / \beta$. The fluctuation of $t_{\max }$ around its mean value is represented by

$$
\sigma_{\alpha} /\langle\beta\rangle \sim 1 \text { r.1. }
$$

because the fluctuation of $\alpha$ is an order of magnitude greater than that of $\beta$. Thus, the shower profile moves event by event about 1 r.1. in longitudinal direction. The positive correlation between $\alpha$ and $\beta$ indicates that a gently rising curve (i.e. with big $\alpha$ ) has a tendency of falling down steeply (i.e. with big $\beta$ ).

The mean values $\mu_{\alpha}(E)$ and $\mu_{\beta}(E)$ were assigned to the fixed terms in eq. (6-5). The fluctuating terms were calculated by the following procedure using $c_{\alpha \beta}, \sigma_{\alpha}$ and $\sigma_{\beta}$.

1. $\sigma_{\alpha}, \sigma_{\beta}$ and $C_{\alpha \beta}$ are calculated as a function of the incident
2. A two by two covariant matrix $M$ is formed by these values.

$$
M=\left[\begin{array}{cc}
\sigma_{\alpha}^{2} & c_{\alpha \beta} \sigma_{\alpha} \sigma_{\beta} \\
c_{\alpha \beta} \sigma_{\alpha} \sigma_{\beta} & \sigma_{\beta}^{2}
\end{array}\right]
$$

3. Eigen values of matrix $M$ are obtained by diagonalizing $M$ by an unitary matrix $U$.

$$
\mathrm{D}=\left[\begin{array}{cc}
\delta_{1}^{2} & 0 \\
0 & \delta_{2}^{2}
\end{array}\right] \quad, \mathrm{D}=\mathrm{U}^{+} \mathrm{MU}
$$

4. Two independent random numbers $\omega_{1 k}$ and $\omega_{2 k}$ are extracted from Gaussian distributions satisfying the following conditions.

$$
\mathbf{w}_{k}=\left[\begin{array}{c}
\omega_{1 k} \\
\omega_{2 k}
\end{array}\right] \quad \begin{array}{ll}
<\omega_{1 k}>=0 & <\omega_{1 k}^{2}>=\delta_{1}{ }^{2} \\
<\omega_{2 k}>=0 & <\omega_{2 k}^{2}>=\delta_{2}{ }^{2}
\end{array}
$$

$$
\ldots(6-9)
$$

5. Transforming these two independent random numbers by the unitary matrix $U$, one obtain the correlated pair of $\Delta \alpha_{k}$ and $\Delta \beta_{k}$ as

$$
\left[\begin{array}{c}
\Delta \alpha_{k} \\
\Delta \beta_{k}
\end{array}\right]=U w_{k}
$$

The covariant matrix $M$ is reproduced by eq. $(6-10)$ as 


$$
\mathrm{M}=\frac{1}{\mathrm{~N}} \sum_{k}\left[\begin{array}{l}
\Delta \alpha_{k} \\
\Delta \beta_{k}
\end{array}\right]\left[\begin{array}{ll}
\Delta \alpha_{k} & \Delta \beta_{k}
\end{array}\right]=\mathrm{UDU}^{+} .
$$

where $N$ is enough larger than 1.

6.2.2 Lateral profile

It is well known that the lateral shower profile has following characteristics [Ref.6-2].

1. It consists of a sharply collimated central component and a wide peripheral part.

2. The spreads become wider as the shower penetrates deeper. As a density function, superposition of two component Gaussian distribution :

$$
P(r)=A N_{1} \exp \left(-\frac{r^{2}}{2 \sigma_{1}^{2}}\right)+(1-A) N_{2} \exp \left(-\frac{r^{2}}{2 \sigma_{2}^{2}}\right)
$$

was selected, where $r$ was distance from the shower center, $\sigma_{1}$ and $\sigma_{2}$ were narrow and wide component of the shower spread in cm, A was the relative intensity of narrow and wide component and $N_{1}$ and $N_{2}$ were the normalization constants.

The $\sigma_{i}(i=1,2)$ was represented by

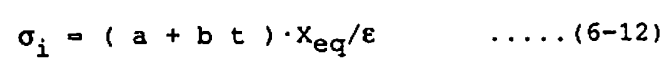

where $t$ was the depth in radiation length, $x_{e q}$ was a physical length per one radiation length of the detector in $\mathrm{cm} / \mathrm{r} .1 ., \varepsilon$ was a critical energy of the material. The constants $a$ and $b$ listed in Table 6-2 were chosen so as to reproduce the beam test results.

\subsection{Comparison with beam test results}

The observed pulse height distribution at each longitudinal segment of the CDF Endplug Electromagnetic Calorimeter and the simulated results are compared in Fig.6-5. The shapes of simulated distribution are consistent with the beam, test results. In order to compare them more quantitatively, the distributions are characterized by following values:

1. mean value of energy fraction, $R_{i}=\left\langle E_{i}>/<E_{\text {total }}>\right.$,

2. normalized fluctuation, $R_{\sigma i}=\sqrt{\left(\left\langle E_{i}{ }^{2}\right\rangle-\left\langle E_{i}\right\rangle^{2}\right)},\left\langle E_{\text {total }}\right\rangle$,

3. correlation factor of energy deposit between the i-th segment and the $j-t h$ segment $c_{i j}$

$$
C_{i j}=\left(\left\langle E_{i} E_{j}\right\rangle-\left\langle E_{i}\right\rangle\left\langle E_{j}\right\rangle\right) / \sqrt{\left(\left\langle E_{i}{ }^{2}\right\rangle-\left\langle E_{i}\right\rangle^{2}\right)\left(\left\langle E_{j}{ }^{2}\right\rangle-\left\langle E_{j}\right\rangle^{2}\right)}
$$

Figure 6-6 shows comparison of these items.

The energy fraction in the first segment decreases with increasing the incident energy while that in the third segment increases. About $90 \%$ of total energy deposit is contained in the second segment, for the electron energies above $40 \mathrm{GeV}$. The observed mean energy fractions were well reproduced by the simulation.

The fluctuations in the first and the second segment decrease with increasing energy because of increasing number of secondary particles. Although the pulse height distributions observed at the third segment and the hadron calorimeter (Fig.6-5) 
are consistent, the simulated fluctuations are less than observed ones. It is considered that observed data points scattered above the main peak in the histograms make the RMS larger. The other fluctuations are in good agreement with the simulation.

The correlation factors $C_{34}$ (correlation factors between the third segment and the hadron calorimeter behind the electromagnetic calorimeter) is positive over all energy region. It means that the both segments have similar tendency of fluctuation. The other correlation factors are small positive values or negative. It indicates that the fluctuation of the electromagnetic shower is compensatory. When a segment has more energy deposit than its average, the other segment have less deposit. The simulated results are not exactly on the data points but their energy dependences were well reproduced.

The observed energy resolutions and simulated one are compared in Fig.6-7. The simulated values are on the measured energy resolution $24 \% / \sqrt{E}$.

Averaged lateral profiles on the pads are compared in Fig.6-8 with beam on the center of the pad as well as their boundary. Observed gain responses along the radial direction are compared with the simulated results as shown in Fig.6-9. Steep falls at. the both inner and outer edge due to the transverse shower leakage are consistent with the simulation.
Chapter 7

Discussions

In preceding Chapters, the performances of the CDF Endplug Electromagnetic Calorimeters and the method of the electromagnetic calorimeter simulation have been described. Now, what are the implications of the electromagnetic calorimeters for physics research? In this chapter, the relation between the performances of electromagnetic calorimeters and the $z^{0}$ mass measurement is discussed with the whole CDF detector simulation program.

7.1 Identification of $2^{0} \rightarrow e^{+} e^{-}$signals

Assuming an integrated luminosity of $10^{37} \mathrm{~cm}^{-2}$, we generated 3000 events of $\mathrm{Z}^{0} \rightarrow \mathrm{e}^{+} \mathrm{e}^{-}$with the Monte Carlo program [Ref.1-18] and processed them with the CDF detector simulation program. A transverse energy flow of a typical event is shown in Fig.7-1. A simultaneous two large electromagnetic energy deposits with balanced transverse momenta is a signature of those events. Transverse momenta of the electron pairs distribute around $\mathrm{M}_{\mathrm{Z}} / 2$ as shown in Fig.7-2. The polar angular distribution of the electron is shown in Fig.7-3. More than $95 \%$ of electrons are contained in $|\eta|<3$. Thus, the CDF calorimeter system has wide enough coverage for them. The number of electrons coming into each calorimeter component or a crack between them are shown in Table 7-1. In the present analysis, we assume no crack filler to be added to original CDF design [Ref.7-1). Both electrons come to the central calorimeter with probability of 198 . About 98 of electron pair hit the endplug calorimeter. Probability which at least one electron 
hits the endplug calorimeter is more than $40 \%$.

The detector simulation program simulated the energy sharing of showering particles among the calorimeter towers. Since the size of the physical towers was different by detectors, energy deposits in physical towers were merged to logical towers, each of which spans $15^{\circ}$ in azimuth and about 0.1 in pseudorapidity.

The electron pair from $z^{0}$ was identified by following procedures.

1. An electromagnetic Et cluster was defined by transverse energy deposit in $3 \times 3$ logical towers (Fig.7-4). The cluster with more than $10 \mathrm{GeV}$ of $\mathrm{Et}$ was picked up.

2. The momentum of a charged particle in a cone spanning the cluster was compared with the electromagnetic energy deposit in it. If the two quantities were consistent with each other within three standard deviations of the calorimeter energy resolution, it was identified as a candidate of an electron cluster (E/P cut).

3. In order to reject overlapping of a $\pi^{ \pm}$and $\pi^{0}$ 's faking the electron signal, clusters which had more than $10 \%$ of electromagnetic energy in the hadron calorimeter were rejected from electron cluster candidates. (Ehad/Eem cut)

7.2 Response of the CDF calorimeter system

7.2.1 Acceptance

Figure 7-5 shows the number of clusters which is obtained by the first selection algorithm described above. More than $80 \%$ of the total number of events were recognized as two clusters.
About 108 of them were classified as zero or one cluster events. Half of these events were due to $10 \mathrm{GeV}$ Et cut of the cluster (Fig.7-2), and the other half were attributed to very little energy deposit in the calorimeter cells because of electrons coming directly into a crack between the calorimeter modules. Some clusters were rejected by the second and the third selection criteria because of the electrons hitting near the boundaries of the calorimeter modules (see the shadowed area in Fig.7-6). These electrons generally made less energy deposits than those hitting middle of the calorimeter cells:

There is an insensitive area spanning $\Delta \theta \sim 2{ }^{\circ}$ at $\theta=90^{\circ}$ in between two arches $\left(90^{\circ}\right.$-crack), which causes loss of $3 \%$ of total events. A crack between two adjacent wedge modules spans $0.6^{\circ}$ in every $15^{\circ}(\phi$-crack $)$. These cracks cause 28 of event loss. Near the boundary between the central calorimeter and the endplug calorimeter at $\theta \sim 30^{\circ}, 108$ of total number of events were lost. Almost the same number of events were lost at $\theta \sim 10^{\circ}$, where was a boundary between the endplug calorimeter and the forward/backward calorimeter. About one third of the total number of events were rejected from the final event sample.

7.2.2 Shower leakage

Simulated calorimeter response were examined by shooting each center of calorimeter cell with 50 and 100 Gev-electrons in the detector simulation program. Figure 7-7 shows the simulated energy deposit in each calorimeter cell. At the boundary of the calorimeter module, $\theta \sim 10^{\circ}$ and $\theta \sim 30^{\circ}$, the response becomes $60 \sim 70 \%$ of $7-3$ 
those in the middle part of the calorimeters. The responses of the central and endplug calorimeter depend on the incident angle of the electron because of shower leakage back to the calorimeter. No significant gain saturation of the forward/backward calorimeter is observed since it has deep enough longitudinal coverage.

The following leakage correction were applied for the simulated energy deposit in each tower:

$$
E_{i}^{\prime}=E_{i}\left(1+a_{i} E_{i}\right),
$$

where $E_{i}$ is the observed energy deposit in the $i-t h$ tower, $a_{i}$ was the correction factor and $E^{\prime}{ }_{i}$ was the corrected energy deposit. As shown in Fig.7-7, the corrected response becomes flat over all angular region except for at the towers near $30^{\circ}$ and $10^{\circ}$ crack.

\subsubsection{Mass resolution of $z^{0}$}

The mass resolution of the electron pair from $z^{0}$ was studied as a function of the energy resolution and the gain uniformity of the calorimeter. In this analysis, it was assumed that the endplug and the forward/backward calorimeter have the same energy resolution and gain uniformities. The opening angle between the electron and the positron from $z^{0}$ was assumed to be known without any uncertainties.

Figure 7-8 shows the mass resolution vs. energy resolution of the calorimeters with an uniform gain. This result suggests that the energy resolution of the central calorimeter is more sensitive to the mass resolution than that of the forward/backward and endplug calorimeters.

With Fixed energy resolution of the central and the endplug+F/B region, $148 / \sqrt{E}$ and $24 \% / \sqrt{E}$, the effect of gain uniformity was studied (Fig.7-9). Since the uncertainty of the energy measurement coming from the energy resolution of the calorimeter is about 28 , the gain nonuniformity less than 18 does not affect the mass resolution.

7.3 Results of fitting the mass peak

Since the observed mass distribution is smeared by the experimental resolution which is almost comparable with the decay width of $z^{0}$, the distribution can not be fitted by a simple Lorentzian. The mass distributions shown in Fig,7-10 were fitted to the function with four free parameters

$$
f(x)=\frac{c \Gamma}{\sigma} \int \frac{\exp \left(-\left(x-x^{\prime}\right)^{2} / 2 \sigma^{2}\right)}{\left(x^{\prime}-M\right)^{2}+\Gamma^{2} / 4} d x^{\prime},
$$

where $M$ and $\Gamma$ were the mass and the decay width of the $z^{0}, \sigma$ was the mass resolution of the detector system and $C$ was a normalization factor.

The results of the fit were summarized in Table 7-2. If the calorimeter system has an uniform gain response, the mass and the decay width for 3000 events of $z^{0} \rightarrow e^{+} e^{-}$is determined within $50 \mathrm{MeV}$ and $170 \mathrm{MeV}$, respectively. In case of the integrated luminosity of $10^{36} \mathrm{~cm}^{-2}$ i.e. $3002^{0} \rightarrow \mathrm{e}^{+} \mathrm{e}^{-}$events, these numbers become $\delta \mathrm{M} \sim 150 \mathrm{MeV}$ and $\delta \Gamma \sim 600 \mathrm{MeV}$.

As discussed before, the gain nonuniformity less than 18 does not affect the precision of the final parameters. If the calorimeter had 38 of its gain nonuniformity, however, the uncertainty of these parameter would be 1.5 times as much as those with uniform gain. 


\subsection{Discussions}

7.4.1 Mass measurements

The Standard Model can be tested by comparing the theoretically predicted parameters such as the radiative correction $\Delta r$ or the mass difference $m_{Z}-m_{W}$ with those obtained by direct measurement of IVB's masses: The uncertainties of the parameters coming from the uncertainty of the mass measurement were studied.

Assuming a general function of the masses, $F\left(m_{Z}, m_{W}\right)$, we can evaluate the effect of statistical error associated with $m_{Z}$ and $m_{W}$ by a usual manner :

$$
\delta F\left(m_{Z}, m_{W}\right)_{\text {stat }}=\sqrt{\left(\partial F / \partial m_{Z}\right)^{2} \delta M_{Z}(s t a t)^{2}+\left(\partial F / \partial m_{W}\right)^{2} \delta m_{W}(s t a t)^{2}} .
$$$$
\cdots(7-3-a)
$$

The relative uncertainty $\delta F / F$ is evaluated as :

$$
\begin{aligned}
(\delta F / F)^{2}= & {\left[E_{w} \text { (stat) }\left(\delta m_{w}(\text { stat }) / m_{w}\right)\right]^{2}+} \\
& {\left[E_{Z} \text { (stat) }\left(\delta m_{Z}(\text { stat }) / m_{Z}\right)\right]^{2}, \quad \ldots(7-q-a) }
\end{aligned}
$$

where $E_{w, z}$ are error propagation coefficients defined by $\left\langle\partial E / \partial m_{W, z}\right) \cdot\left(m_{W, z} / F\right)$.

The effect of the systematic uncertainties of the IVB's masses have strong correlation coming from the absolute energy calibration of the calorimetry so that the uncertainty of the function becomes :

$$
\delta F\left(m_{Z}, m_{W}\right)_{s y s}=1 \partial F / \partial m_{W} \delta m_{W}(s y s) \quad 1, \quad \ldots(7-3-b)
$$

where the ratio $m_{Z} / m_{W}$ is assumed to be constant in the function $F$. Similarly as eq. (7-4-a), the relative uncertainty is evaluated as: $\delta F / F=\mid E_{W}(s y s) \cdot\left\langle\delta \mathrm{m}_{W}(\right.$ sys $\left.) / \mathrm{m}_{W}\right) \mid$

$\cdots(7-4-b)$

where $E_{W}$ (sys) is defined by $E_{W}($ sys $)=\left(\partial F / \partial m_{W}\right) \cdot\left(m_{W} / F\right)$.

Five parameters, the mass difference $m_{W}-m_{Z}$, the radiative correction factor $\Delta x$ defined in eq. (1-5), the weak mixing angle defined by $\sin ^{2} \theta_{W}=1-\left(m_{W} / m_{Z}\right)^{2}$ and $\sin ^{2} \theta_{W}=\left(A / m_{W}\right)^{2}$ and the $\rho$ parameter were selected as the function $F$. The error propagation coefficients $E_{W, z}$ of these five parameters were numerically evaluated as in Table 7-3 where the IVB's masses were set to be $m_{W}$ $=81.2 \mathrm{GeV}$ and $\mathrm{m}_{2}=92.5 \mathrm{GeV}$. Table $7-3$ shows that the $p$ parameter can be determined with the best accuracy for a given uncertainty of the measured masses while the $\Delta r$ has the largest uncertainty in these parameters.

The UA1 and UA2 collaborations have reported their results on the IVB's mass measurement (Ref.7-2,3). The five parameters defined above are calculated by using their masses as in Table 7-4. Their results are in good agreement with the theoretical prediction [Ref.1-14]. At the improved version of CERN Antiproton Collider (ACOL), it is planned to collect an integrated luminosity fLdt $10 \mathrm{pb}^{-1}$ which corresponds to about $5000 \mathrm{w} \rightarrow \mathrm{ev}$ events and 500 $z \rightarrow e^{+} e^{-}$events. In this case, the statistical uncertainties are almost as same amount as the systematic ones as in Table 7-4.

In the CDF, the statistical uncertainty of $m_{z}$ was estimated to be $\delta \mathrm{m}_{\mathrm{z}}$ (stat) $-0.05 \mathrm{GeV}$ with an integrated luminosity of $10^{37}$ $\mathrm{cm}^{-2}$. The systematic uncertainty of absolute energy calibration in 7-7 
the CDF calorimetry will be about 18. The statistical uncertainty of $\mathrm{m}_{\mathrm{w}}$ was assumed to be the same as $\mathrm{m}_{\mathrm{Z}}$ as seen in Refs.7-2 and Ref.7-3. By taking the masses obtained by UA2 collaboration [Ref.7-3] and the expected systematic and statistical uncertainties in the CDF, the contribution of these parameters were evaluated as shown in Table 7-4.

The statistical uncertainty is the main contribution to these parameters in the UA2 and UA1 experiments, while it is not a substantial uncertainty in the CDF. In such cases, if the absolute energy calibration is performed with the accuracy of $1 \%$, the mass difference, for example, is determined within relative uncertainty of 1\%. Thus the absolute energy calibration is one of the most important subject for testing the Standard Model in the CDF.

According to eq. (1-1), the mass difference is given by a function of $\sin \theta_{W}$ as illustrated in Fig. 7-11. Current results and the expected value in the CDF are plotted in Fig.7-11. Small uncertainty of $\sin ^{2} \theta_{W}$ and the mass difference in the CDF will make it possible to see the deviation of the data point from the theoretical prediction without the precise knowledge of $m_{2}$ itself.

Figure 7-12 shows the $m_{t}$ dependence of the mass difference with results obtained by the UAI and the UA2 collaborations. Because of the uncertainty of the theoretical prediction of the mass difference $\sim 0.5 \mathrm{GeV}$, the current results can specify neither upper nor lower bounds of the $\mathrm{m}_{t}$. In case of the CDF shown in Fig. 7-12, it may be possible to determine the upper or lower bound of $m_{t}$. However, it will be hard to determine $m_{t}$ itself by this method, because of the theoretical uncertainty of the mass difference prediction.

\subsubsection{Decay width of $\mathrm{z}^{0}$}

According to the Ref.7-4, the relation between the mass resolution $\sigma$ and the uncertainty of the decay width $\delta \Gamma$ is given by the following formula

$$
\delta \Gamma=[2 / \mathrm{N}]^{1 / 2}\left[\Gamma^{2}+(2.35 \sigma)^{2}\right]^{1 / 2}, \ldots(7-5)
$$

where $\mathrm{N}$ is the number of observed events. The $\delta \Gamma$ is shown in Fig. $7-13$ as a function of the mass resolution $\sigma$. Our results on $\delta \Gamma$ is almost consistent with eq. (7-5). If the integrated luminosity is more than $10^{37} \mathrm{~cm}^{-2}$ and the nonuniformity of the calorimeter system is less than $1 \%$, the uncertainty of the number of additional neutrino species will be less than 1 2. 


\section{Chapter 8}

Conclusions

CDF Endplug Electromagnetic Calorimeter were designed and constructed under strict quality control to realize uniform responses.

The performances of the calorimeter were tested with a high energy electron beam with similar operation condition as final colliding beam experiment at Fermilab.

The energy resolution was $248 / \sqrt{E}$ and nonlinearity was 88 for 200 Gev-electrons. Uniformity of the calorimeter response was better than 98 besides the peripheral region.

studying the shower profile observed by the calorimeter, we established the realistic electromagnetic calorimeter simulation program as a part of $\mathrm{CDE}$ detector simulation program.

The performances of the calorimeter were evaluated as a function of $z^{0}$ mass and decay width measurements with the detector simulation program. If the integrated luminosity is more than $10^{37} \mathrm{~cm}^{-2}$ and the calorimeters have quite uniform gain, the statistical uncertainties of mass and the decay width become $50 \mathrm{MeV}$ and $170 \mathrm{MeV}$ respectively.

The uncertainties of the Standard Model parameters at the CDF given as functions of the IVB's masses will be reduced to one tenth of the current results obtained by the UA1 and the UA2 collaborations. The uncertainty of the additional number of neutrino families determined by measuring the decay width of $z^{0}$ at the CDF is estimated to be $1 \sim 2$.

The gain nonuniformity of the calorimeter worse than the nominal energy resolution, which is about 2 in case of the CDF, affects the uncertainty of these parameters. Thus, the gain nonuniformity should be kept within 28 . 


\begin{tabular}{|c|c|c|c|c|c|}
\hline & Hans $B$ Tensen & IEEE NS SYMP & & $(1985)$ & \\
\hline$[1-2]$ & $\mathrm{CDF}$ group & CDF Design Repo: & & (1981) & \\
\hline$[1-3]$ & S.Mori et al. & Nucl. Inst. Meth. & A238 & (1985) & 18 \\
\hline$[1-4]$ & L. Nodulman et al. & Nucl. Inst. Meth. & 204 & (1983) & 351 \\
\hline$[1-5]$ & w.c.Carithers et al. & 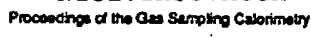 & nertop 11, Forts & (1985) & \\
\hline$[1-6]$ & G.Brandenburg et al. & 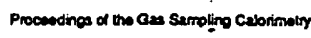 & Workestoo II, Farmitate & (1985) & \\
\hline 7] & Bagnaia P. et al. & Phys. Lett. & $122 B$ & (1983) & 273 \\
\hline$[1-7]$ & G.Arnison et al. & Phys. Lett. & $129 \mathrm{~B}$ & (1983) & 273 \\
\hline$[1-7\}$ & G.Arnison et al. & Phys. Lett. & $122 \mathrm{~B}$ & (1983) & 103 \\
\hline$[1-8]$ & G. Arnison et al. & Phys. Lett. & $134 \mathrm{~B}$ & (1983) & 496 \\
\hline ] & Bagnaia p.et al. & Phys. Lett. & $129 \mathrm{~B}$ & (1983) & 130 \\
\hline$[1-9]$ & G.Arnison et al. & Phys. Lett. & 1268 & (1983) & 398 \\
\hline$[1-10]$ & G. Arnison et al. & Phys. Lett. & $147 \mathrm{~B}$ & (1984) & 241 \\
\hline$[1-11]$ & S.Geer & CERN-EP & 163 & (1985) & \\
\hline$[I-11]$ & J.A.Appel et al & CERN-EP & 166 & & \\
\hline$[1-12]$ & S. Weinberg & Phys. Rev. Lett & 19 & (1967) & 1264 \\
\hline$[1-13\}$ & A. Sirlin & Pys. ReV. & D22 & $(15$ & 971 \\
\hline$[1-14]$ & M.J.Marciano et al. & Phys. Rev & D29 & 4) & 945 \\
\hline$[1-15]$ & B. Lee et al. & Phys. Rev. Lett & 38 & 7) & 383 \\
\hline$[1-16]$ & D. Albert et al. & Nucl. Phys. & B166 & & 460 \\
\hline$[1-17]$ & Kenichi Hi\} & Phys. Rev. & D29 & 4) & 1939 \\
\hline$[1-18]$ & H. -U. Beng & p. Phys. Comn. & 31 & & 323 \\
\hline$[1-18]$ & T. Sjostra & .Phys .Comm. & 27 & 2) & 243 \\
\hline$[1-18]$ & T. Sjostra & & 28 & & 227 \\
\hline$[1-18]$ & H. $-U$. Bengtsson & Comp.Phys . Comn. & 31 & (1984) & 323 \\
\hline$[1-19]$ & M.N.Minard & KEK Report & $85-5$ & & \\
\hline$[1-19]$ & G. Arnison et al & Phys. Lett. & 1478 & (1984) & 493 \\
\hline$[1-20]$ & R. Odorico et al. & IFUB $(84 / 18)$ & 18 & (1984) & \\
\hline$[1-21]$ & Horst D. Wahl & CERN-EP $(84 / 127)$ & 127 & & \\
\hline$(1-22)$ & H.Miyata et al. & IEEE & NS -31 & (1 & 74 \\
\hline$[1-23]$ & H. Miyata & TPP & 17 & & \\
\hline$[1-23]$ & R. Engelmann et al. & 1. Inst. Meth. & 216 & 3) & 45 \\
\hline$[1-24]$ & Y.Hayashide et al & h. & 22 & & 452 \\
\hline$[1-24]$ & K.Fujii et al. & st. Meth. & A236 & (15 & 55 \\
\hline$[1-24]$ & Y.Hayashide et al. & Nucl. Inst. Meth. & 204 & & 361 \\
\hline$[1-25]$ & H.G.Fisher & Nucl. Inst. Meth. & 156 & (1978) & 81 \\
\hline$[2-1]$ & Y.Hayashide e & Nucl. Inst. Meth. & 216 & 3) & 127 \\
\hline$[3-1]$ & Y.. Fukui, Y.Hayashide & $\mathrm{CDF}$ & 267 & 115 & \\
\hline$[4-1]$ & & CD & 266 & & \\
\hline & oege & $\mathrm{CD}$ & 11 & (1) & \\
\hline & ii et al. & $C D$ & 26 & & \\
\hline$[5-1]$ & R.L.Anderson et a & IEEE & NS -25 & & 340 \\
\hline & M.Z & IE & NS - 28 & 119 & 500 \\
\hline$[5-3]$ & J.J. Mueller et al. & IEEE & NS-28 & (1981) & 496 \\
\hline$[5-4]$ & Abarbro et al. & Nucl. Inst. Meth. & 213 & & 223 \\
\hline & R.Fabrizio et al. & Nucl. Inst. Meth. & 227 & (1984) & 220 \\
\hline & H.Videau et & Nucl. In & 225 & (1984) & 481 \\
\hline$[6-1]$ & R.L. Ford & SLAC & 210 & (1978) & \\
\hline
\end{tabular}

$\begin{array}{llllrr}\text { [6-2] } & \text { G. Bathow et al. } & \text { Nucl. Phys. } & \text { B20 } & (1970) & 592 \\ \text { [7-1] } & \text { J.w. Cooper et al. } & \text { CDF } & 289 & (1985) & \\ \text { [7-2] } & \text { G. Armison et al. } & \text { Phys. Lett. } & 1668 & (1986) & 484 \\ \text { [7-3] } & \text { J.A.Appel et al. } & \text { 2. Phys. } & \text { C30 } & (1986) & 1 \\ \text { [7-4] } & \text { S. Aronson et al. } & \text { DO DESIGN REPORT } & & (1984) & \end{array}$


Table captions

Table 1-1 Characteristics of IVB's measured by CERN UAl and UA2 collaboration (Ref. 1-11).

Table 1-2 Contribution of top quark mass to the radiative correction factor $\Delta r$ (Ref. 1-14).

Table 1-3 QCD corrected decay rate of IVB's quoted from Ref.1-15.

Table 1-4 Results on IVB masses and electroweak interaction parameters measured by electron channels and muon channels quoted from Ref.1-21.

Table 2-1 Configuration of longitudinal pad segments and location of strip electrodes.

Table 5-1 Energy resolution of gas sampling calorimeters.

Table 6-1 Final parameters of longitudinal shower profile for CDF electromagnetic calorimeter simulation.

Table 6-2 Parameters of lateral shower development.

Table 7-1 Acceptance of the total electron pair from $z^{0}$ decay for calorimeters or cracks between them.

Table 7-2-a Mass and decay width obtained by the fit for 300 $z^{0} \rightarrow e^{+} e^{-}$events under different gain uniformity of the calorimeter.

Table 7-2-b Mass and decay width obtained by the fit for 3000 $z^{0} \rightarrow e^{+} e^{-}$events under different gain uniformity of the calorimeter.

Table 7-3 Error propagation coefficents of the Standard Model parameters.
Table 7-4 Comparison of statistical and systematic uncertainties of the Standard Model parameters. The UA1 and UA2 results are based on the current data reported in [7-2] and [7-3]. Errors of CDF are estimated by assuming the integrated luminosity of $10^{37} \mathrm{~cm}^{-2}$. 
Fig.1-1 Tracks of the $\bar{p} p$ collision at the center of mass energy of $1.6 \mathrm{TeV}$ observed by Vertex TPC of the CDF central detector system in 1985 .

Fig.1-2 The CDF detector system consisting of the central, the forward and the backward detectors.

Fig.1-3 Elevation view of the CDE central detector system.

Fig.1-4 A wedge module of the central calorimeter.

Fig.1-5 Elevation view of the CDF forward/backward detector system.

Fig.1-6 The IVB's mass difference $m_{Z}-m_{W} v s . m_{Z}$. The solid curve includes radiative corrections while the dashed curve is uncorrected. [Ref.1-14]

Fig.1-7 Self-energy, tadpole, and counterterm contributions to muon decay. [Ref.1-13]

Fig.1-8 Production cross sections of $\mathrm{w}^{ \pm}$and $\mathrm{z}^{0}$ as a function of $\sqrt{s}$ calculated by LUND Monte Carlo.

Eig.1-9 Energy distribution of electron from $2^{0}$ decay in angular region of central, endplug and forward/backward calorimeter.

Fig.2-1 Location of the CDF endplug electromagnetic calorimeter.

Fig.2-2 Structure of the gas sealed vessel for the CDF Endplug Electromagnetic Calorimeter.

Fig.2-3 Structure of a proportional chamber consisting of proportional tube array with conductive plastic tubes, pick-up electrode and plane or strip G-10 sheets.

Figure captions - 1
Fig.2-4

Close view of proportional counters each consisting of a $7 \mathrm{~mm} \times 7 \mathrm{~mm}$ conductive plastic tube, a $50 \mu \mathrm{m}$ gold plated tungsten wire and two tube endcaps supporting the anode wire.

Fig.2-5-a Tower geometry of CDF endplug electromagnetic calorimeter. Each tower has three longitudinal segments.

Fig.2-5-b Strip electrode pattern placed near the shower maximum to get finer $\theta$ position information ( $\theta$ strip pattern).

Fig.5-5-c $\phi$ strip pattern.

Fig.2-6 Induced cathode signal along the anode wire vs. resistivity of the tube.

Fig.3-1 Setup of the bench test system. Anode signal induced by $\mathrm{Fe}^{55}$ radio active source was read out by Lecroy 2285 ADC via a preamplifier LeCroy TRA1000.

Fig.3-2 Gas gain vs. wire diameter.

Fig.3-3 Gas gain variation vs. wire displacement from the center of the tube.

Fig.3-4 Production process of the proportional chamber.

Fig.3-5 Assembling jig to keep straightness of the proportional tubes during the curing time of the glue.

Eig.3-6 Block diagram of automatic anode wire tension measurement system.

Fig.3-7 Configuration of cosmic ray test system. The cosmic ray was triggered by upper and lower hodoscope trigge counters. Gas gain was monitored by a monitor tube with $\mathrm{Fe}^{55}$ source during the data taking.

Figure captions - 2 
Configuration of charge sensitive amplifier card,

Fig. 3-8

Examples of pulse height distribution induced by cosmic ray signals. They were fitted to Landau distributions.

Fig.3-9 Distribution of gain variation in a chamber $\Delta G$ observed by the cosmic ray test. It is defined by gain variation in RMS over mean response within a chamber.

Fig.3-10 Block diagram of the pad checking system consisting of a pulse generator and Aan DC both controlled by a micro computer.

Fig.3-11 Pulse height observed by the pad checking system. Although it depends on the size of the pad, bad connections were clearly distinguished from good ones.

Fig.3-12. Thickness distribution of a lead sheet measured by an ultrasonic thickness gauge.

Fig.4-1 Arrangement of momentum tagging system at Fermilab M-bottom beam line.

Fig.4-2 Momentum distribution of 100 GeV-electrons. The momentum spread was 2 3\% for $100 \mathrm{GeV}$-electron beam.

Fig.4-3 Configuration of rotating stand control system.

Fig.4-4 Scanning points. Each center of pad was shot by $100-G e V$ electron beam.

Fig.4-5 View of a RABBIT Hutch.

Fig.4-6 Configuration of the readout system at M-bottom beam line. Analog signals amplified by charge sensitive amplifiers were digitized by an EWE module. Digitized 16 bit data were transfered to the host computer via CAMAC-RABBIT interfaces.
CARROT. It consists of 24 channels of charge sensitive amplifiers, self-calibration circuit and fast out circuit for trigger.

Fig.4-8 Timing chart of the sample and hold circuit on the CARROT card. The pulse height of amplifier output was sampled before and after the beam crossing. In the beam test, the before gate was generated by a clock generator and the after gate was defined by various kinds of trigger.

Fig.4-9 Structure of the CAMAC-RABBIT interface. A $32 \mathrm{kbit}$ static RAM stores data read from $E W E$ and the host computer. The I/O procedure is controlled by the sequencer.

Fig.4-10 Gas gain variation observed by gas gain monitor tubes in 1984. Maximum variation was 18 an hour.

Fig.4-11 Gas gain vs. gas pressure. The temperature was kept constant during this measurement.

Fig.4-12 Gas gain monitoring system at M-bottom beam line. Six monitor tubes were installed per quadrant. They were read out by LeCroy 2285 ADC via TRA1000 preamplifier.

Fig.4-13 Data acquisition system at M-bottom beam line. Serial and parallel CAMAC line were connected to Jorway 411 branch driver. The Bison Box accepted the triggers and interrupted the host computer.

Fig.4-14 Trigger system at M-bottom beam line consisting of beam, pedestal, pad check and source trigger logic.

Fig.4-15 Cable connection checking system. The anode wires of the chambers were pulsed by pulser via the high voltage distributer. Induced signal on the pads were read out. 
Fig.5-1 Total pulse height distribution of anode wire signal for $100 \mathrm{GeV}$-electrons.

Fig.5-2 Energy resolution of wire and pad signal at various. operational voltages.

Fig.5-3 Pulse height vs. operational voltage for $100 \mathrm{GeV}$ and $160 \mathrm{GeV}$-electrons.

Fig.5-4 Linearity of anode wire and pad signal at various operational voltages. The line was determined lowest two data points and origin of the figure.

Fig.5-5 Linearity curve at an operational voltage of $1.80 \mathrm{kV}$. These points were fitted to eq. (5-2). The gradient of the line is a derivative of eq. $(5-2)$ at $E=0$.

Fig.5-6 The averaged longitudinal shower profiles and their fitted curve.

Fig.5-7 The energy dependence of the shower parameter $\alpha$ and $\beta$.

Eig.5-8 Estimated longitudinal shower leakage vs, incident ellectron energy.

Fig.5-9-a Estimated longitudinal shower leakage vs. incident angle $\theta$. The leakage increase steeply at $\theta=32^{\circ}$ because of limited diameter of the calorimeter.

Fig.5-9-b Closer view of Fig.5-9-a. The leakage becomes $6 \frac{8}{a}$ at $\theta=12$ - for $200 \mathrm{GeV}$-electrons.

Fig.5-10 Number of layers vs. energy resolution.

Fig.5-11-a Pulse height observed by each proportional chamber at low operational voltage $1.70 \mathrm{kV}$ (horizontal axis) and medium operational voltage, $1.80 \mathrm{kV}$ (vertical axis). All plots are on a line. It means the gas gain saturation at every chamber is almost same.

Figure captions - 5
Fig.5-11-b Pulse height at higher operational voltage, $1.90 \mathrm{kV}$ was used instead of the medium voltage. The gas gain saturation seems to depend on the charge density of the shower.

Fig.5-12 Three dimensional view of averaged lateral shower spread.

Fig.5-13 Comparison of parametrized lateral spread with data.

Fig.5-14 Energy dependence of lateral spread parameter. No significant energy dependence of the parameters were found.

Fig.5-15 Comparison of the simulated strip signal and the data.

Fig.5-16 Position resolution vs. electron energy. The strip provide best position resolution $(\sim 2 \mathrm{~mm})$ in both direction.

Fig.5-17 Mean energy fraction in each longitudinal segment. The second segment contain more than 908 above $40 \mathrm{GeV}$.

Fig.5-18-a Pulse height distribution of the gas gain monitor tube signal (main peak of $\mathrm{Fe}^{55}$ source).

Fig.5-18-b Gas gain monitor signal distorted by noise.

Fig.5-19 Distributions of the reduced $\chi^{2}$ and $\sigma / \mu$ of the Gaussian fit.

Fig.5-20 Gas gain at each calibration point. One calibration point corresponds to about one minute.

Fig.5-21 Run by run gas gain variation. One run nominally took 20 minutes.

Fig.5-23 Three dimensional view of the calorimeter response. Figure captions - 6 
Fig.5-24-a Calorimeter resopnse as a function of $\phi$ at various $\theta$.

Fig.5-24-b Calorimeter resopnse as a function of $\theta$ at various $\phi$.

Fig.5-25 Response variation with longitudinal leakage correction.

Fig.5-26-a Calorimeter resopnse as a function of $\phi$ at various $\theta$ with longitudinal leakage correction.

Eig.5-26-b Calorimeter resopnse as a function of $\theta$ at various $\phi$ with longitudinal leakage correction.

Fig.5-27-a Calorimeter resopnse as a function of $\phi$ at the inner or outer edge.

Eig.5-27-b Calorimeter resopnse as a function of $\theta$ near $\phi$-crack.

Fig.6-1 Event by event longitudinal shower profiles for 100 GeV-electrons and their fitted curve.

Fig.6-2 Distributions of $\alpha$ and $\beta$ for 100 GeV-electrons.

Fig.6-3 Energy dependence of $\mu_{\alpha}, \sigma_{\alpha}, \mu_{\beta}$ and $\sigma_{\beta}$. $\mu_{\alpha}$ shows clear In (E) dependence.

Fig.6-4 Scatter plot of event by event $\alpha$ vs. $\beta$. They have strong positive correlation.

Fig.6-5 Comparison of simulated pulse height distribution at each segment with beam test data. Dashed histogram is obtained by the simulation and data points with error bar are the beam test results.
Fig.6-6-a Comparison of simulated mean energy fraction in each longitudinal segment with beam test data.

Fig.6-6-b Comparison of simulated fluctuation of pulse height with observed data.

Fig.6-6-c Comparison of simulated correlation coefficient with observed data.

Fig.6-7 Comparison of simulated energy resolution with observed data.

Fig.6-8 Comparison of simulated transverse energy deposit with data.

Fig.6 -9 Comparison of simulated calorimeter response along the radius vs. observed one.

Fig.7-1 An example of simulated transverse energy flow of $z^{0} \rightarrow e^{+} e^{-}$decay.

Fig.7-2 Transverse momentum distribution of electron pair from $z^{0}$ decay.

Eig.7-3 Polar angular distribution of electron from $z^{0}$ decay. The horizontal axis is in pseudorapidity.

Eig.7-4 Logical and physical tower sizes in $\eta-\phi$ plane.

Fig.7-5 Number of recognized clusters per events.

Fig.7-6 The shadowed towers are located near the boundary of calorimeter modules.

Fig.7-7 Simulated calorimeter response for $100 \mathrm{GeV}$ and 50 GeV-electron beam on the detector simulation program.

Fig.7-8-a $z^{0}$ mass resolution vs. energy resolution of the central 
calorimeters.

Fig.7-8-b $z^{0}$ mass resolution vs. energy resolution of the forward/backward and the endplug calorimeters.

Fig.7-9-a $2^{0}$ mass resolution vs. gain uniformity of the central calorimeter.

Fig.7-9-b $z^{0}$ mass resolution vs. response uniformity of the forward/backward and the endplug calorimeter.

Fig.7-10-a simulated mass distribution of $300 \mathrm{z}^{0} \rightarrow \mathrm{e}^{+} \mathrm{e}^{-}$events with uniform gain of the calorimeter.

Fig.7-10-b simulated mass distribution of $300 \mathrm{z}^{0} \rightarrow \mathrm{e}^{+} \mathrm{e}^{-}$events with gain nonuniformity of $2 \%$.

Fig.7-10-c simulated mass distribution of $3000 \mathrm{z}^{0} \rightarrow \mathrm{e}^{+} \mathrm{e}^{-}$events with uniform gain of the calorimeter.

Fig.7-10-d Simulated mass distribution of $3000 \mathrm{z}^{0} \rightarrow \mathrm{e}^{+} \mathrm{e}^{-}$events with gain nonuniformity of $2 \%$.

Fig.7-11 $\mathrm{m}_{Z}-\mathrm{m}_{\mathrm{W}}$ vs. $\sin ^{2} \theta_{\mathrm{W}}$. Error bars of UA1 and UA2 results are calculated from current data reported in Ref.7-1 and 7-2. The theoretical prediction is shown in Ref. $1-14$.

Fig.7-12 IVB's mass difference $m_{2}-m_{W}$ vs. top quark mass $m_{t}$.

Fig.7-13 The mass resolution vs, uncertainty of $z^{0}$ decay width $\delta \Gamma$. The curve is based on Ref.7-4. The plots are obtained by our analysis. 
UAI group

UA2 group

Number of event

$(w \rightarrow e v)$

172

$O(\mathrm{PP} \rightarrow W \rightarrow e V) 546 \mathrm{GeV}$

$0.55 \pm 0.09 \pm 0.09$

(nb)

$\sigma(P P \rightarrow W \rightarrow e V)_{630 ~ G e V} \quad 0.63 \pm 0.05 \pm 0.09$

$0.06 \pm 0.05$

$[n b]$

$\mathrm{H}_{\mathrm{H}}$

$83.5^{+1.1}-1.0^{ \pm 2.8}$

$81.2 \pm 1.1 \pm 1.3$

$\left[\mathrm{GeV} / \mathrm{c}^{2}\right]$

$r_{W}$

less than 6.5

$\left[\mathrm{GeV} / \mathrm{c}^{2}\right\}$

Number of event

$\left(Z \rightarrow \mathrm{e}^{+} \mathrm{e}^{-}\right)$

14

16

$\sigma(\mathrm{PP} \rightarrow \mathrm{Z} \rightarrow$ ee $) 546 \mathrm{GeV} \quad 40 \pm 20 \pm 6$

$110 \pm 39 \pm 9$

[pb]

$\sigma(\mathrm{PP} \rightarrow \mathrm{Z} \rightarrow$ ee $) 630 \mathrm{GeV} \quad 79+21 \pm 12$

[pb]

$\left[\mathrm{GeV} / \mathrm{c}^{2}\right]$

$r_{2}$

[GeV/c $\left./ c^{2}\right]$

119

$0.50 \pm 0.09 \pm 0.05$

$52 \pm 19 \pm 4$

$92.5 \pm 1.3 \pm 15$

\begin{tabular}{|c|c|c|c|}
\hline$m_{t}(\mathrm{GeV})$ & $\Delta r$ & $A(\mathrm{GeV})$ & $\begin{array}{c}\text { Predicted } \\
m_{Z}-m_{W}(\mathrm{GeV}) \\
\text { for } m_{Z}=93.8 \mathrm{GeV}\end{array}$ \\
\hline 20 & 0.0699 & 38.66 & 10.79 \\
\hline 36 & 0.0696 & 38.65 & 10.79 \\
\hline 60 & 0.0714 & 38.69 & 10.82 \\
\hline 83 & 0.0623 & 38.50 & 10.66 \\
\hline 100 & 0.0558 & 38.37 & 10.56 \\
\hline 150 & 0.0379 & 38.01 & 10.27 \\
\hline 200 & 0.0167 & 37.60 & 9.96 \\
\hline 240 & -0.00355 & 37.21 & 9.68 \\
\hline $\begin{array}{l}\text { Tree } \\
\text { approximation }\end{array}$ & 0 & 37.28 & 9.73 \\
\hline
\end{tabular}

$2.19^{+0.7}-0.5 \pm 0.22$

Table 1- 2

Table 1-1 


\begin{tabular}{|c|c|c|c|c|c|}
\hline $\sin ^{2} \theta_{w}$ & 0.21 & 0.22 & 0.23 & 0.24 & 0.25 \\
\hline 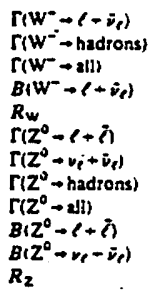 & $\begin{array}{l}0.239 \\
2.44 \\
3.22 \\
0.080 \\
9.33 \\
0.095 \\
0.185 \\
2.36 \\
3.20 \\
0.030 \\
0.058 \\
24.9\end{array}$ & $\begin{array}{l}0.242 \\
2.29 \\
3.02 \\
0.080 \\
9.43 \\
0.089 \\
0.176 \\
2.21 \\
3.01 \\
0.030 \\
0.058 \\
24.8\end{array}$ & $\begin{array}{l}0.226 \\
2.13 \\
2.81 \\
0.080 \\
9.13 \\
0.083 \\
0.168 \\
2.09 \\
2.85 \\
0.030 \\
0.059 \\
24.6\end{array}$ & $\begin{array}{l}0.212 \\
2.00 \\
2.64 \\
0.080 \\
9.43 \\
0.080 \\
0.160 \\
1.96 \\
2.68 \\
0.030 \\
0.060 \\
24.4\end{array}$ & $\begin{array}{l}0.200 \\
1.88 \\
2 . .48 \\
0.081 \\
9.43 \\
0.077 \\
0.154 \\
1.85 \\
2.54 \\
0.030 \\
0.061 \\
24.1\end{array}$ \\
\hline
\end{tabular}

Table 1- 3
Results on IVB masses and electroweak interaction parameters

\begin{tabular}{|c|c|c|c|}
\hline & \multicolumn{2}{|l|}{ UAl } & \multirow{2}{*}{$\frac{\text { UA2 }}{e}$} \\
\hline & e & $\mu$ & \\
\hline mw & $80.9 \pm 1.5 \pm 2.5$ & $\begin{array}{r}81.0+6.0 \\
-7.0\end{array}$ & $83.1 \pm 1.9 \pm 1.3$ \\
\hline $\mathrm{mz}_{\mathrm{z}}$ & $95.6 \pm 1.4 \pm 2.9$ & $\begin{array}{r}85.8+7.0 \\
-5.4\end{array}$ & $92.7 \pm 1.7 \pm 1.4$ \\
\hline $\sin ^{2} \theta_{w}^{21}$ & $0.284 \pm 0.040$ & $\begin{array}{r}0.103+0.212 \\
-0.165\end{array}$ & $0.196 \pm 0.047$ \\
\hline $\sin ^{2} \theta_{w} b$ & $0.228 \pm 0.008 \pm 0.014$ & $\begin{array}{r}0.228+0.040 \\
-0.033\end{array}$ & $0.216 \pm 0.010 \pm 0.007$ \\
\hline e & $0.93 \pm 0.05$ & $\begin{array}{r}1.15+0.22 \\
-0.28\end{array}$ & $1.02 \pm 0.06$ \\
\hline
\end{tabular}

Table $1-4$ 


\section{LONGITUDINAL SEGMENTATION}

logngltudinal segments

chamber \# readout electrodes
configurations

radiation length

segment sum Incluoing
steal from plate
0.72 r.l.

the first pad segment

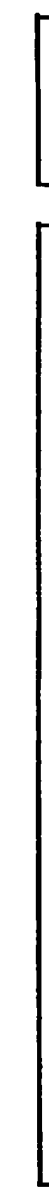

the second pad segment

1
2
3
4
-5

$2.55 \quad 3.27$

$\theta$-STRIP

$\phi$ STRIP

$\theta$-STRIP

QSTRIP

$\theta$-STRIP

STRIP

$\theta$-STRIP

${ }_{\phi}$ STRIP

-STRIP

$\phi$ STRIP

12.2415 .51
- proportional mode operation

* Geiger mode operation

Table 5-1

Eneray resolutions of

Gas Sampling Electromagnetic Calorimeters

\begin{tabular}{|l|c|c|c|c|}
\hline ENERGY RANGE & $\begin{array}{c}\sigma / \mu \sqrt{ } E \\
\% \cdot \sqrt{G e V}\end{array}$ & $\begin{array}{c}\sigma / \mu \sqrt{ } \text { E/t } \\
\% \cdot \sqrt{G e V} \cdot r .1\end{array}$ & $\begin{array}{c}\text { SAMPLING } \\
\text { THICKNESS } \\
\text { r.I. }\end{array}$ & REFERENCE \\
\hline $0.5-15 \mathrm{GeV}$ & 17 & 24 & $\mathrm{~Pb} 0.5$ & $5-1$ \\
\hline $10-46 \mathrm{GeV}$ & 24 & 27 & $\mathrm{~Pb} 0.8$ & $5-2$ \\
\hline $0.5-3.2 \mathrm{GeV}$ & 17 & 35 & $\mathrm{~Pb} 0.23$ & $5-3$ \\
\hline $0.5-15 \mathrm{GeV}$ & $\begin{array}{l}17 \\
12\end{array}$ & $\begin{array}{c}38 \\
27\end{array}$ & $\mathrm{~Pb} 0.2$ & $5-4$ \\
\hline $0.2-1.84 \mathrm{GeV}$ & 17 & 24 & $\mathrm{~Pb} 0.5$ & $5-5$ \\
\hline $0.5-3.2 \mathrm{GeV}$ & 16 & 27 & $\mathrm{~Pb} 0.35$ & $5-6$ \\
\hline $0.5-4 \mathrm{GeV}$ & 21 & 29 & $\mathrm{~Pb} 0.51$ & $5-7$ \\
\hline
\end{tabular}

$2.55 \quad 18.06$ 
Acceptoance of electron pair from $Z^{\grave{2}}$ decay

$\mu_{\alpha}=2.4+0.56$ In (E)
$\sigma_{\alpha}=0.5$
$\mu_{\beta}=0.5$
$\sigma_{\beta}=0.05$
Table 6-1
$\sigma_{1} \quad 0$.
$\sigma_{2} \quad 8.19$
$A_{1}=0.6$
Table 6-2

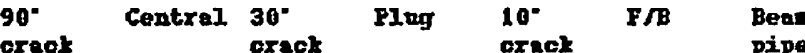

\begin{tabular}{|c|c|c|c|c|c|c|}
\hline & oreok & crack & & cracl & & pipe \\
\hline $\begin{array}{l}90^{\circ} \\
\text { crack }\end{array}$ & 0.6 & 0.1 & 0.6 & 0.2 & 0.2 & 0.0 \\
\hline Central & 20.2 & 7.0 & 27.0 & 6.9 & 10.1 & 0.7 \\
\hline $\begin{array}{l}30^{\circ} \\
\text { crack }\end{array}$ & & 0.5 & 4.7 & 1.0 & 1.7 & 0.0 \\
\hline Plug & & & 8.3 & 4.1 & 4.7 & 0.1 \\
\hline $\begin{array}{l}10^{\circ} \\
\operatorname{crack}\end{array}$ & & & & 0.4 & 0.7 & 0.0 \\
\hline F/B & & & & & 0.2 & $\begin{array}{l}0.0 \\
0.0\end{array}$ \\
\hline
\end{tabular}

Beam
pipe 


\begin{tabular}{|c|c|c|c|c|c|}
\hline \multicolumn{2}{|c|}{$\begin{array}{l}\text { Uniformity of central } \\
\text { calorimeter }\end{array}$} & 1008 & \multirow[t]{2}{*}{998} & \multirow[t]{2}{*}{988} & 978 \\
\hline & $\begin{array}{l}\text { formity of } F / B \\
\text { orimeter }=1008\end{array}$ & & & & \\
\hline M & $(\mathrm{GeV})$ & 93.95 & 93.88 & 93.93 & 93.81 \\
\hline$\delta \mathrm{M}$ & $(\mathrm{GeV})$ & .05 & .05 & .05 & .06 \\
\hline$\Gamma$ & $(\mathrm{GeV})$ & 2.67 & 2.91 & 2.71 & 3.25 \\
\hline & $(\mathrm{GeV})$ & .17 & .18 & .19 & .24 \\
\hline \multicolumn{6}{|c|}{ Uniformity of $F / B$} \\
\hline & $(\mathrm{GeV})$ & 93.95 & 93.95 & 93.91 & 93.83 \\
\hline$\delta M$ & $(\mathrm{GeV})$ & .05 & .05 & .05 & .06 \\
\hline$\Gamma$ & $(\mathrm{GeV})$ & 2.61 & 2.88 & 2.98 & 2.12 \\
\hline & $(\mathrm{GeV})$ & .19 & .19 & .2 & .21 \\
\hline \multicolumn{6}{|c|}{$\begin{array}{l}\text { Uniformity of } F / B \\
\text { calorimeter }=988\end{array}$} \\
\hline & $(\mathrm{GeV})$ & 93.91 & 93.95 & 93.85 & 93.88 \\
\hline & $(\mathrm{GeV})$ & .05 & .05 & .06 & .06 \\
\hline$\Gamma$ & (GeV) & 2.61 & 2.83 & 2.6 & 2.51 \\
\hline & $(\mathrm{GeV})$ & .19 & .20 & .20 & .22 \\
\hline \multicolumn{6}{|c|}{$\begin{array}{l}\text { Uniformity of } \mathrm{F} / \mathrm{B} \\
\text { calorimeter }=978\end{array}$} \\
\hline$M$ & $(\mathrm{GeV})$ & 93.95 & 93.90 & 93.91 & 93.88 \\
\hline$\delta M$ & $(\mathrm{GeV})$ & .05 & .06 & .06 & .06 \\
\hline$\Gamma$ & $(\mathrm{GeV})$ & 2.89 & 2.85 & 2.67 & 2.47 \\
\hline$\delta \Gamma$ & $(\mathrm{GeV})$ & .20 & .22 & .23 & .26 \\
\hline
\end{tabular}

\begin{tabular}{|c|c|c|c|c|c|}
\hline \multicolumn{2}{|c|}{$\begin{array}{l}\text { Uniformity of central } \\
\text { calorimeter }\end{array}$} & $100 \%$ & 998 & 988 & 978 \\
\hline \multicolumn{6}{|c|}{$\begin{array}{l}\text { Uniformity of F/B } \\
\text { calorimeter }=1008\end{array}$} \\
\hline & & 93.68 & 93.76 & 93.92 & 93.75 \\
\hline & $(\mathrm{GeV})$ & .15 & .15 & .17 & .18 \\
\hline$\Gamma$ & $(\mathrm{GeV})$ & 2.56 & 2.87 & 2.61 & 2.16 \\
\hline & $(\mathrm{GeV})$ & .59 & .52 & .64 & .81 \\
\hline \multicolumn{6}{|c|}{$\begin{array}{l}\text { Uniformity of } F / B \\
\text { calorimeter }=99 \%\end{array}$} \\
\hline$M$ & $(\mathrm{GeV})$ & 93.69 & 93.68 & 94.01 & 93.81 \\
\hline & $(\mathrm{GeV})$ & .16 & .15 & .17 & .18 \\
\hline$\Gamma$ & $(\mathrm{GeV})$ & 2.80 & 2.80 & 1.71 & 2.67 \\
\hline & $(\mathrm{GeV})$ & .56 & .51 & .58 & .81 \\
\hline \multicolumn{6}{|c|}{$\begin{array}{l}\text { Uniformity of } F / B \\
\text { calorimeter }=988\end{array}$} \\
\hline$M$ & $(\mathrm{GeV})$ & 93.99 & 93.92 & 93.65 & 93.83 \\
\hline$\delta \mathrm{M}$ & $(\mathrm{GeV})$ & .15 & .17 & .18 & .18 \\
\hline$r$ & $(\mathrm{GeV})$ & 2.61 & 2.83 & 2.6 & 2.51 \\
\hline & $(\mathrm{GeV})$ & .61 & .79 & .72 & .74 \\
\hline \multicolumn{6}{|c|}{$\begin{array}{l}\text { Uniformity of } F / B \\
\text { calorimeter }=978\end{array}$} \\
\hline M & $(\mathrm{GeV})$ & 94.07 & 93.92 & 93.781 & 94.07 \\
\hline$\delta M$ & $(\mathrm{GeV})$ & .17 & .19 & .20 & .19 \\
\hline$\Gamma$ & $(\mathrm{GeV})$ & 2.45 & 3.08 & 2.52 & 3.03 \\
\hline$\delta \Gamma$ & $(\mathrm{GeV})$ & .63 & .80 & 1.01 & .74 \\
\hline
\end{tabular}

$3000 z^{0} \rightarrow e^{+} e^{-}$

$300 \mathrm{z}^{0} \rightarrow \mathrm{e}^{+} \mathrm{e}^{-}$ 


\begin{tabular}{lrcc}
$F$ & EW[stat.] & Ez [stat.] & E[sys.] \\
\hline$m_{Z}-m_{W}$ & 7.19 & 8.19 & 1.00 \\
$\Delta r$ & 53.46 & 76.12 & 22.66 \\
$\sin ^{2} \theta_{W}=1-\left(m_{W} / m_{Z}\right)^{2}$ & 6.72 & 6.72 & - \\
$\sin ^{2} \theta_{W}=\left(A / m_{W}\right)^{2}$ & 2.00 & 0.00 & 2.00 \\
$\rho$ & 1.41 & 2.00 & 0.59 \\
\hline
\end{tabular}

$\mathrm{em}_{\mathrm{W}}=81.2 \mathrm{GeV}$

$\mathrm{m}_{\mathrm{Z}}=92.5 \mathrm{GeV}$

$\delta F[\text { stat. }]^{2}=E_{W}$ (stat. $]^{2} \cdot\left(\delta m_{W}[\text { stat. }] / m_{W}\right)^{2}$

$+E_{\mathrm{Z}}[\text { stat. }]^{2} \cdot\left(\delta \mathrm{m}_{\mathrm{Z}}\right.$ [stat. $\left.] / \mathrm{m}_{\mathrm{Z}}\right)^{2}$

$\delta F_{\text {[sys. }]}=\mid E_{W, z}\left[\right.$ sys.] $\cdot\left(\delta m_{W, z}\left[\right.\right.$ sys.] $\left./ m_{W, z}\right) \mid$

Table 7-3

\begin{tabular}{|c|c|c|c|c|}
\hline Table 7-4 & Theory & UA1 & UA2 & $\mathrm{CDE}$ * \\
\hline $\mathbf{m}_{\mathbf{W}}$ & 83.0 & 83.50 & 81.20 & 81.20 \\
\hline$\delta m_{W}[$ stat.] & \pm 2.90 & \pm 1.10 & \pm 1.10 & \pm 0.05 \\
\hline$\delta \mathrm{m}_{W}[$ stat. $]$ (ACOL) & & \pm 0.204 & \pm 0.170 & \\
\hline$\delta \mathrm{m}_{W}[$ sys.] & & \pm 2.70 & \pm 1.30 & \pm 0.81 \\
\hline $\mathbf{m}_{\mathbf{z}}$ & 93.8 & 93.00 & 92.50 & 92.50 \\
\hline$\delta m_{z}$ [stat.] & \pm 2.40 & \pm 1.40 & \pm 1.30 & \pm 0.05 \\
\hline$\delta \mathrm{m}_{\mathrm{Z}}[$ stat. $]$ (ACOL) & & \pm 0.250 & \pm 0.233 & \\
\hline$\delta \mathrm{m}_{\mathrm{z}}$ [sys.] & & \pm 3.00 & \pm 1.50 & \pm 0.93 \\
\hline$m_{z}-m_{W}$ & 10.8 & 9.50 & 11.30 & 11.30 \\
\hline$\delta\left(m_{Z}-m_{W}\right)[$ stat. $]$ & \pm 0.50 & \pm 1.78 & \pm 1.70 & \pm 0.07 \\
\hline$\delta\left(m_{Z}-m_{W}\right)[$ stat. $]$ (ACOL) & & \pm 0.320 & \pm 0.286 & \\
\hline$\delta\left(m_{z}-m_{W}\right)[$ sys.] & & \pm 0.31 & \pm 0.18 & \pm 0.11 \\
\hline$\Delta \mathbf{r}$ & 0.0696 & -0.028 & 0.081 & 0.081 \\
\hline$\delta(\Delta r)[$ stat. $]$ & \pm 0.002 & \pm 0.155 & \pm 0.105 & \pm 0.004 \\
\hline$\delta(\Delta r)[$ stat. $]$ (ACOL) & & \pm 0.028 & \pm 0.018 & \\
\hline$\delta(\Delta r)[$ sys.] & & \pm 0.066 & \pm 0.029 & \pm 0.018 \\
\hline $\sin ^{2} \theta_{W}=1-\left(m_{W} / m_{Z}\right)^{2}$ & 0.217 & 0.194 & 0.229 & 0.229 \\
\hline$\delta\left(\sin ^{2} \theta_{W}\right)[$ stat. $]$ & \pm 0.014 & \pm 0.032 & \pm 0.030 & \pm 0.0013 \\
\hline$\delta\left(\sin ^{2} \theta_{\mathrm{W}}\right)[$ stat. ] (ACOL) & & \pm 0.006 & \pm 0.005 & \\
\hline$\delta\left(\sin ^{2} \theta_{W}\right)[$ sys.] & & \pm 0.000 & \pm 0.000 & \pm 0.0000 \\
\hline $\sin ^{2} \theta_{W}=\left(A / m_{W}\right)^{2}$ & 0.217 & 0.214 & 0.227 & 0.227 \\
\hline$\delta\left(\sin ^{2} \theta_{W}\right)[$ stat. $]$ & \pm 0.014 & \pm 0.006 & \pm 0.006 & \pm 0.0003 \\
\hline$\delta\left(\sin ^{2} \theta_{W}\right)[$ stat. $](\mathrm{ACO})$ & & \pm 0.001 & \pm 0.001 & \\
\hline$\delta\left(\sin ^{2} \theta_{W}\right)[$ sys.] & & \pm 0.014 & \pm 0.007 & \pm 0.0045 \\
\hline$p$. & 1 & 1.026 & 0.996 & 0.996 \\
\hline$\delta p$ [stat.] & \pm 0.000 & \pm 0.037 & \pm 0.034 & \pm 0.002 \\
\hline$\delta p[$ stat. ] (ACOL) & & \pm 0.007 & \pm 0.006 & \\
\hline$\delta \rho$ [sys.] & & \pm 0.018 & \pm 0.009 & \pm 0.006 \\
\hline
\end{tabular}

References

Theory : $[1-14]$, UA1 : [7-2], UA2 : $[7-3]$

* UA2 results are assumed for the mean values, and errors are estimated by assuming the integrated luminosity of $10^{37} \mathrm{~cm}^{-2}$. 


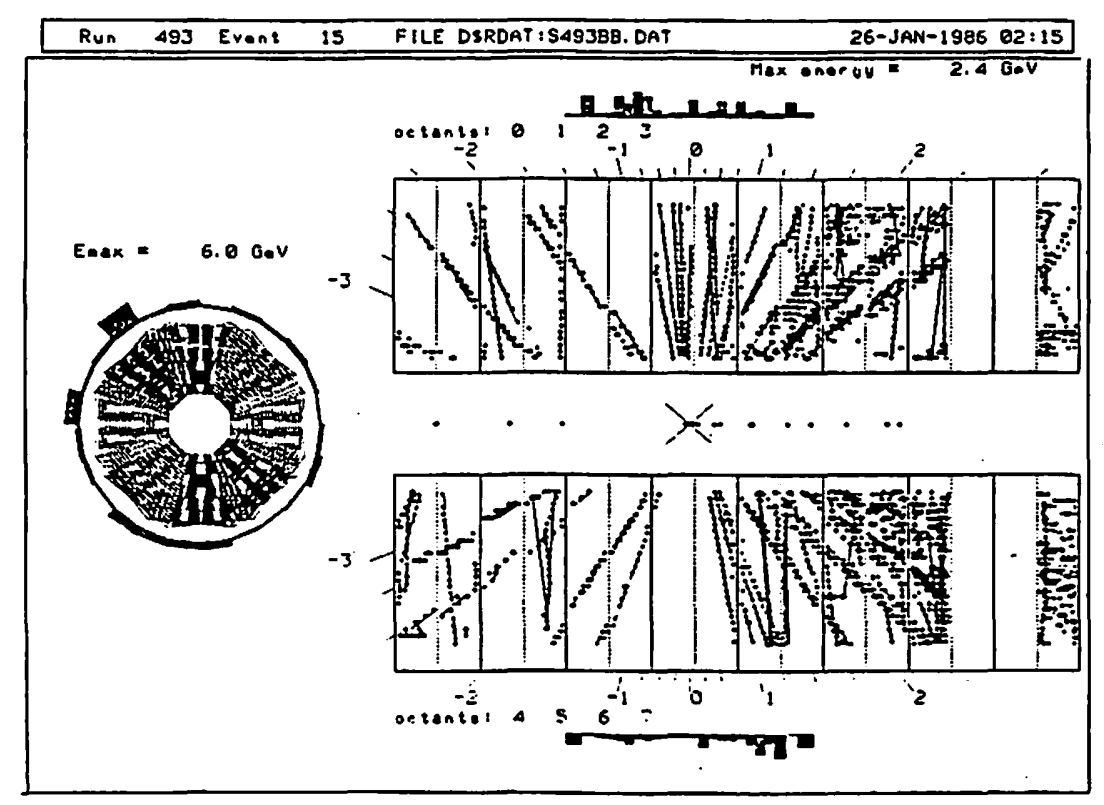

Fig.1-1

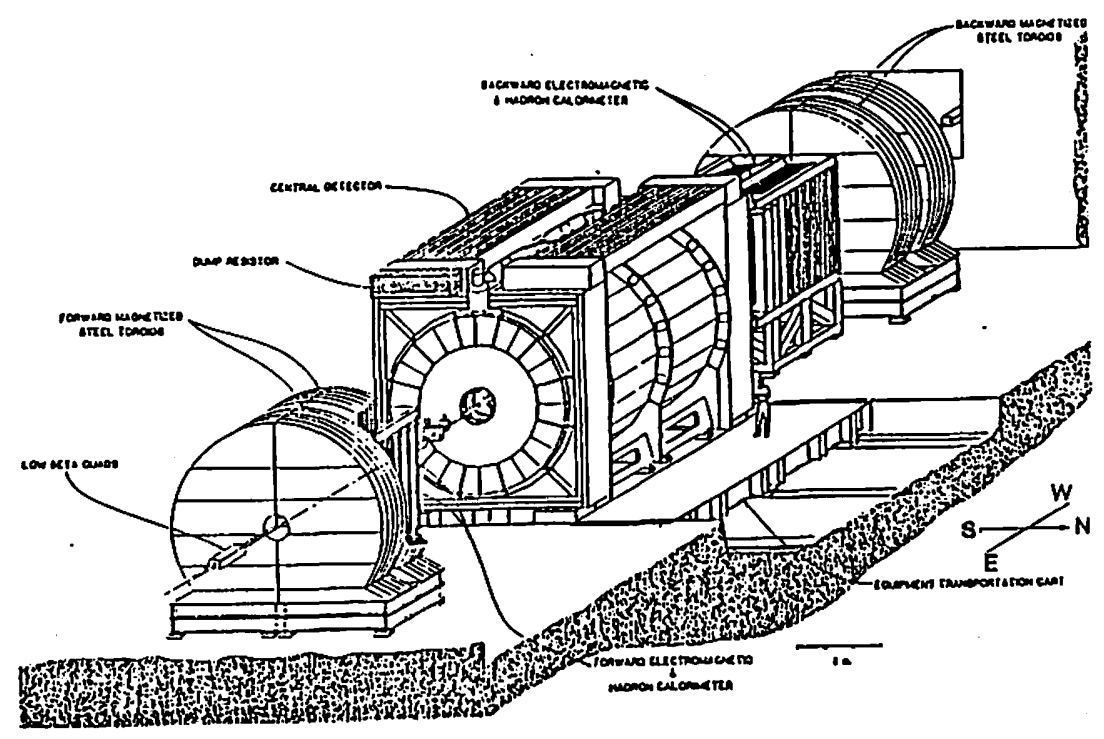

Fig. 1- 2 


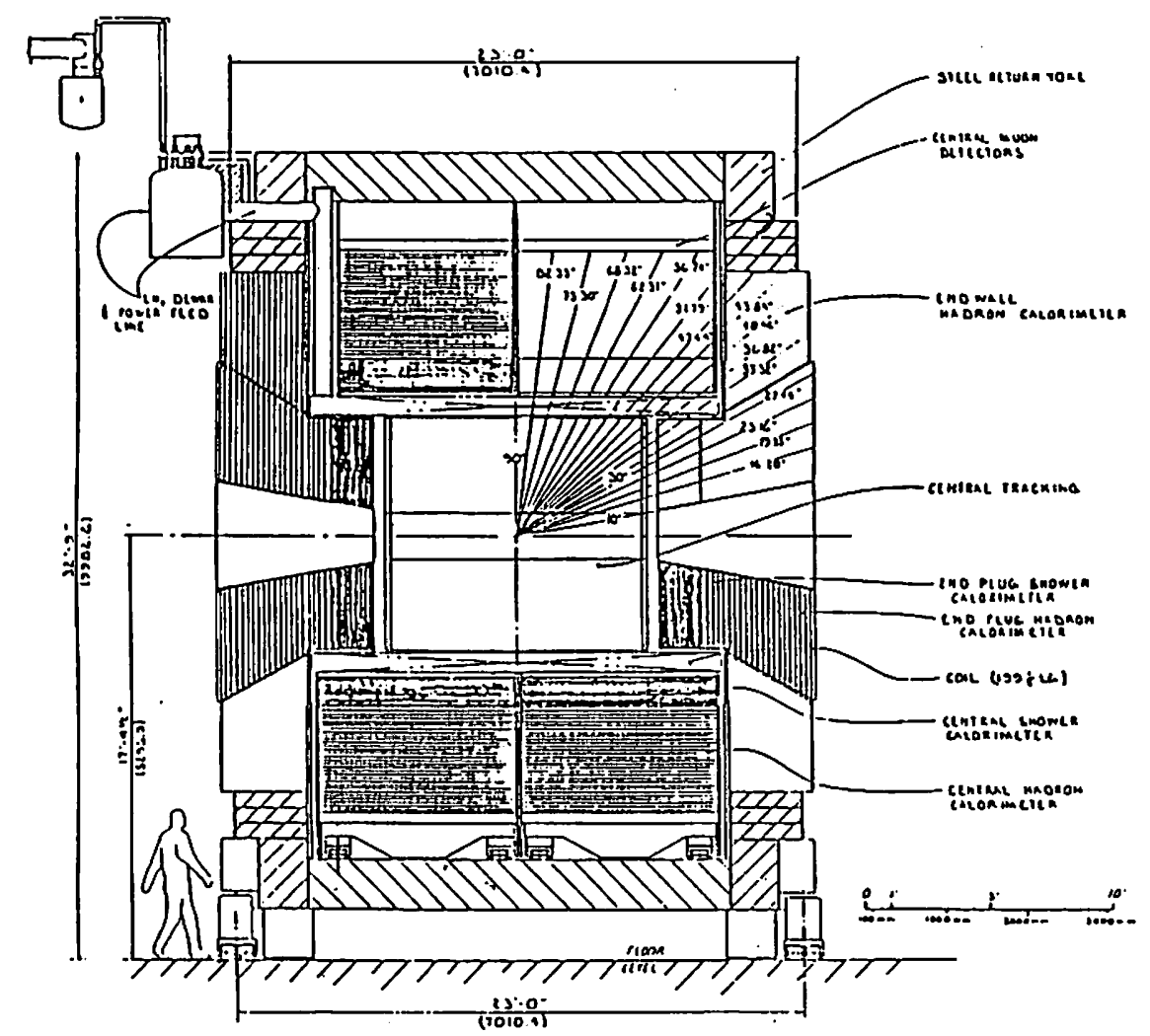

Plg. 1- 3

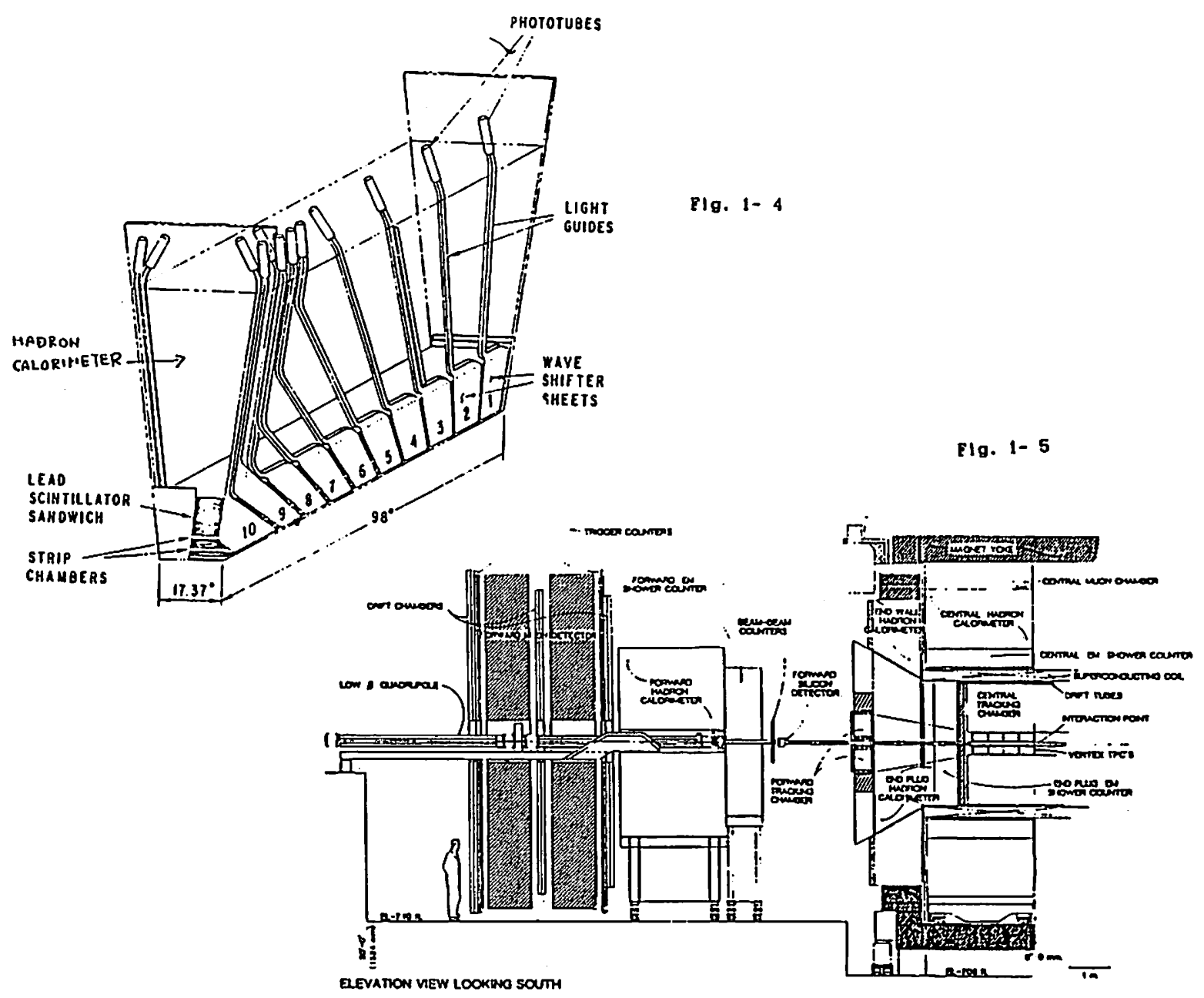




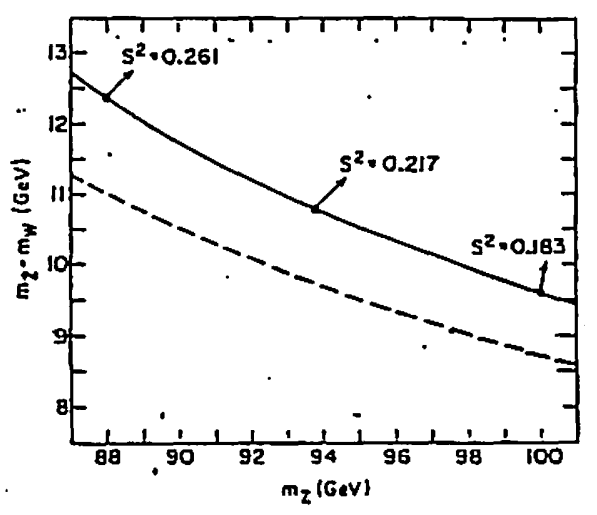

Fig. $1-6$
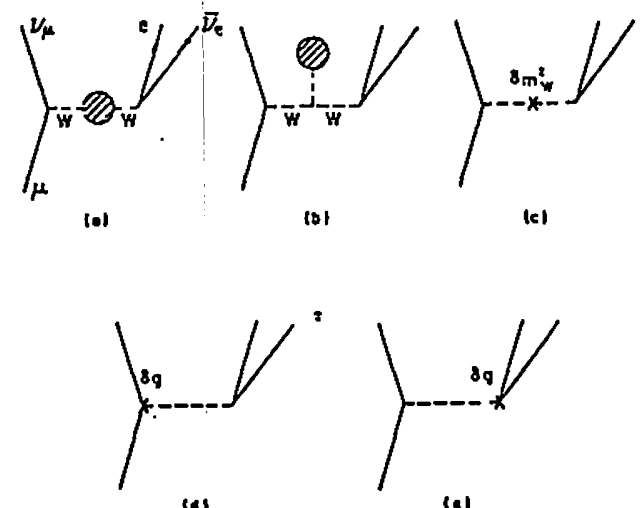

(1)

(a)

Fig. 1- 7 

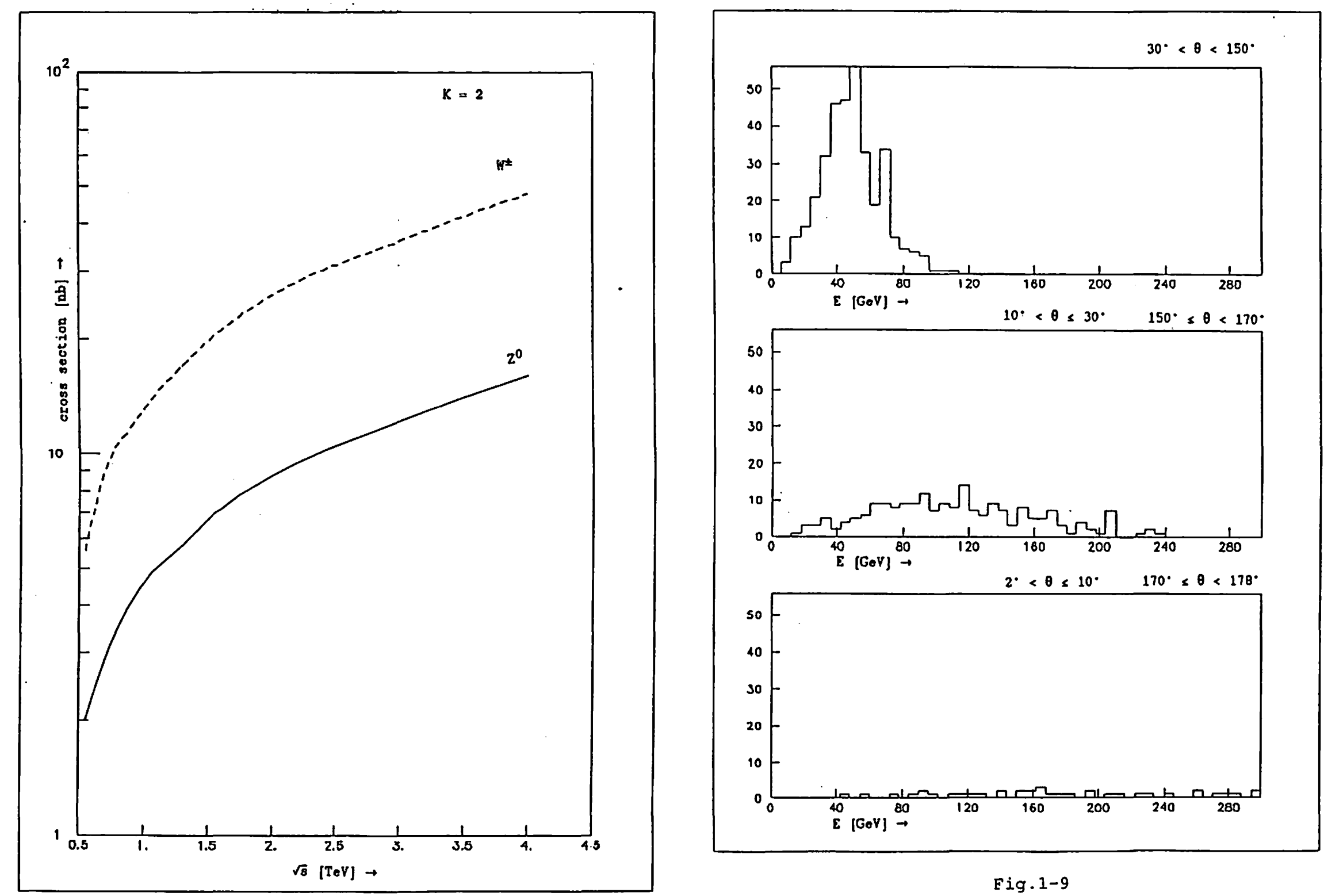

Fig.1-9

Fig.1-8. 


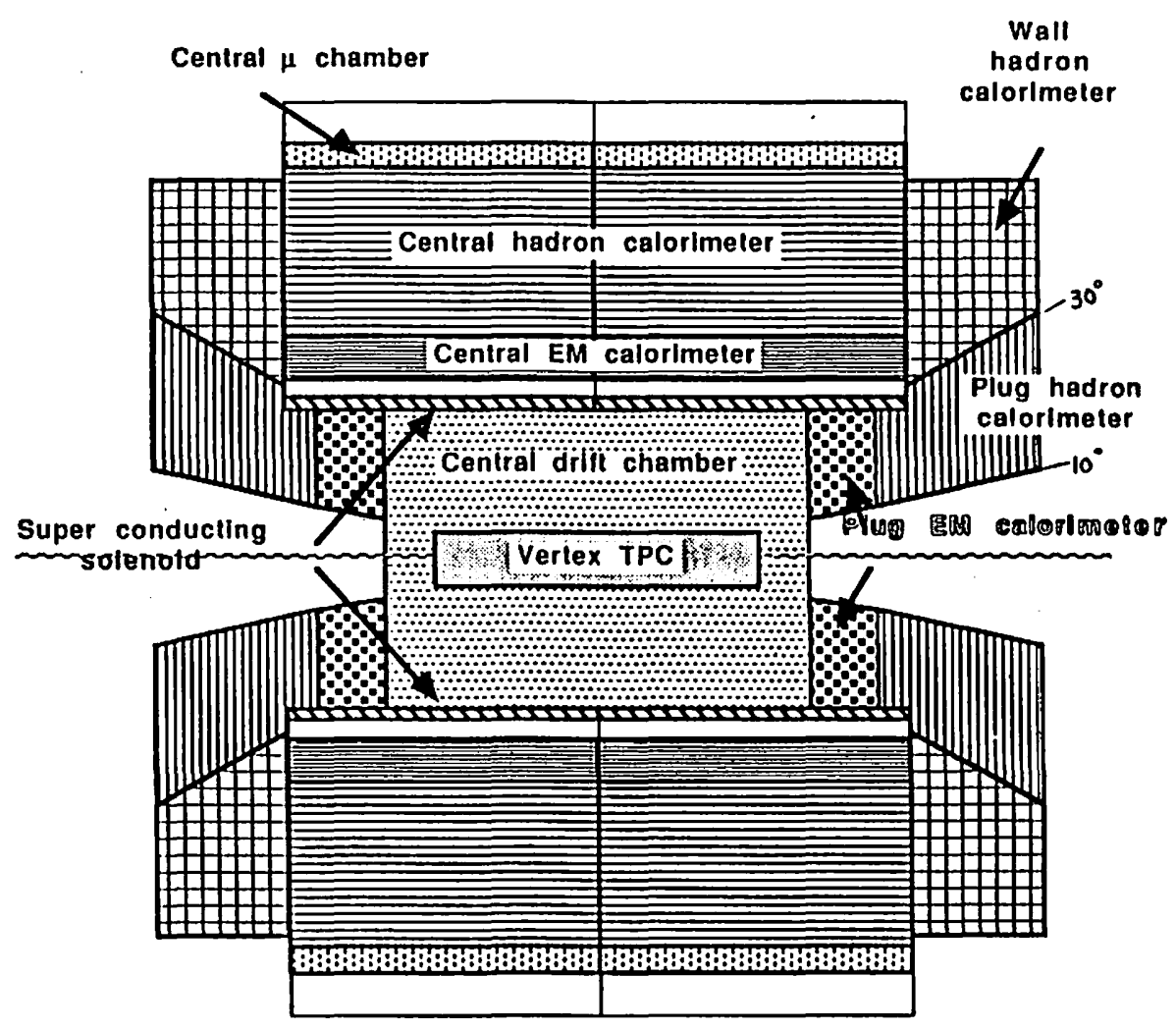

Fig.2-1

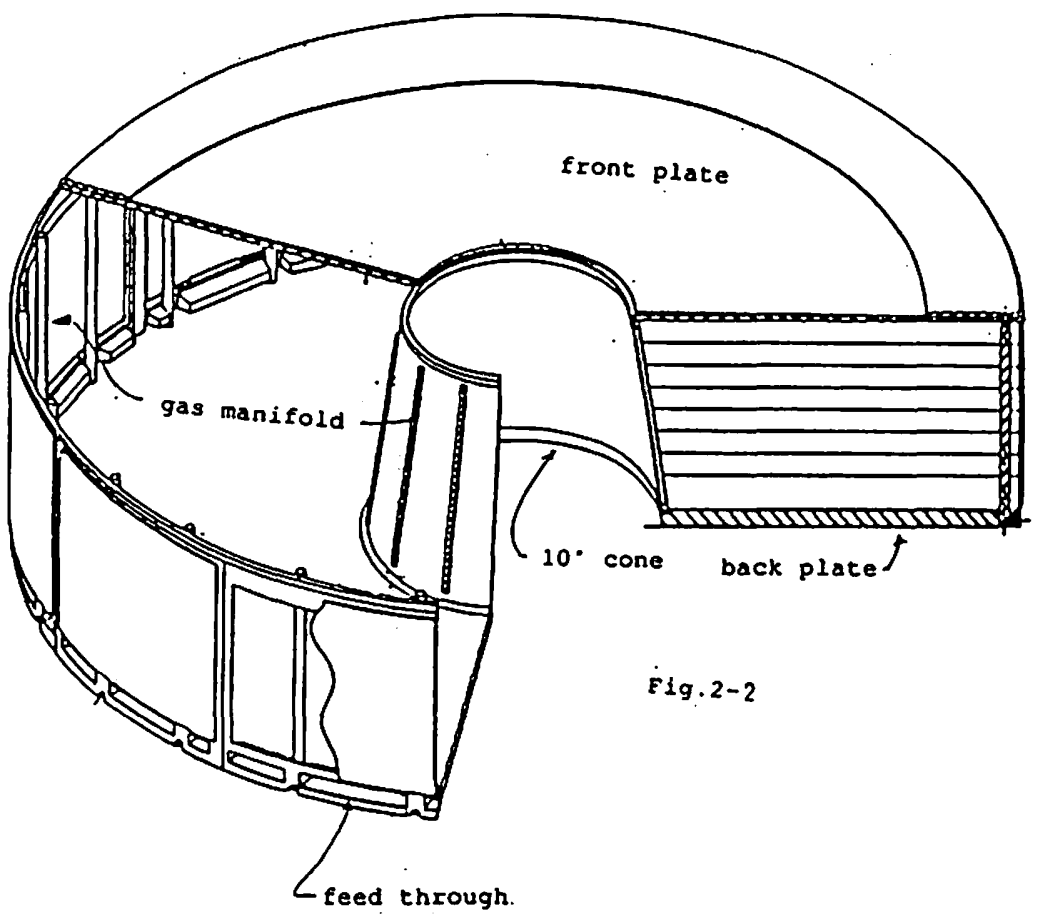




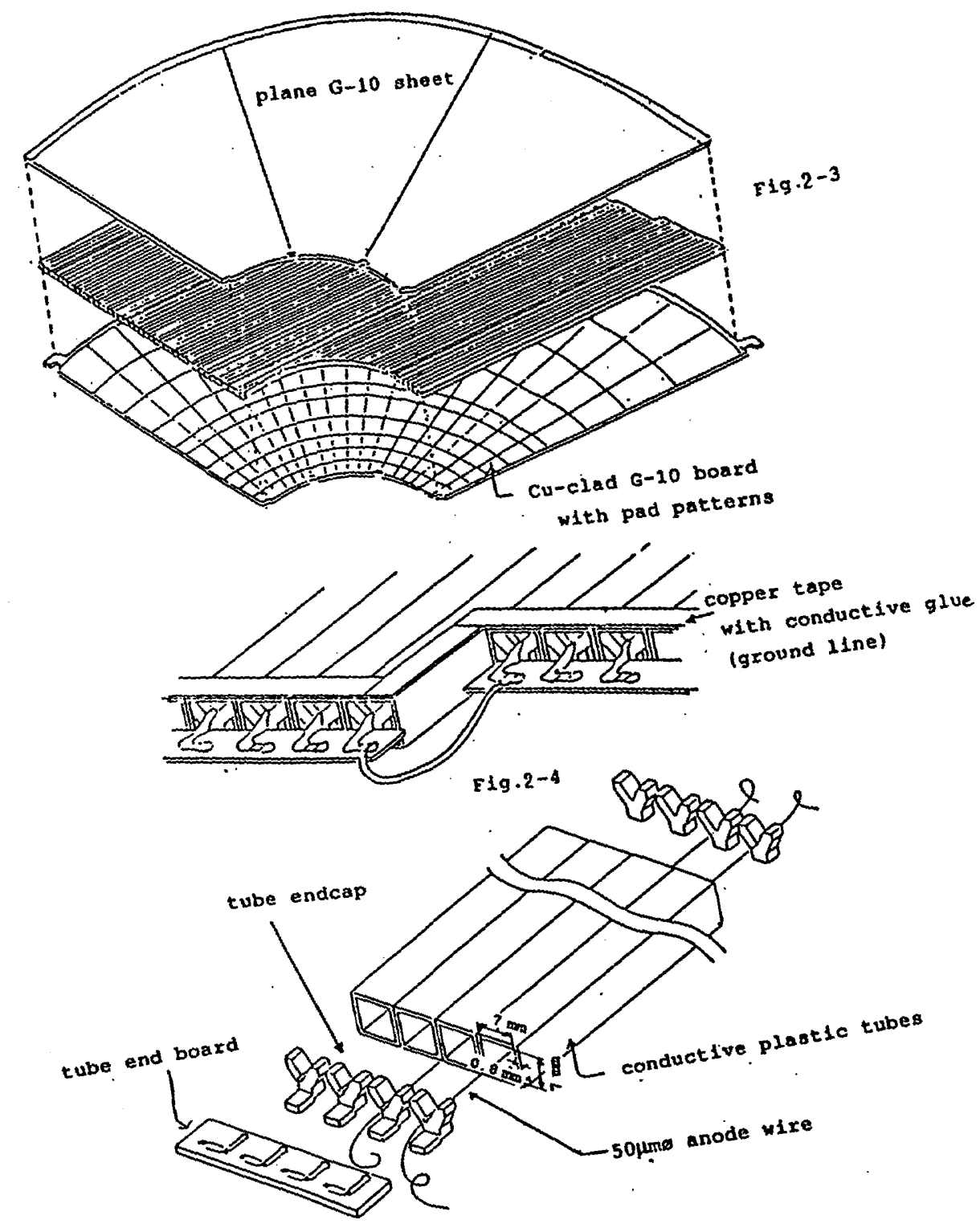


Strip Pattern

Tower Geometry of CDE

Endplug Electromagnetic Calorimeter

\section{longitudinal gegments}

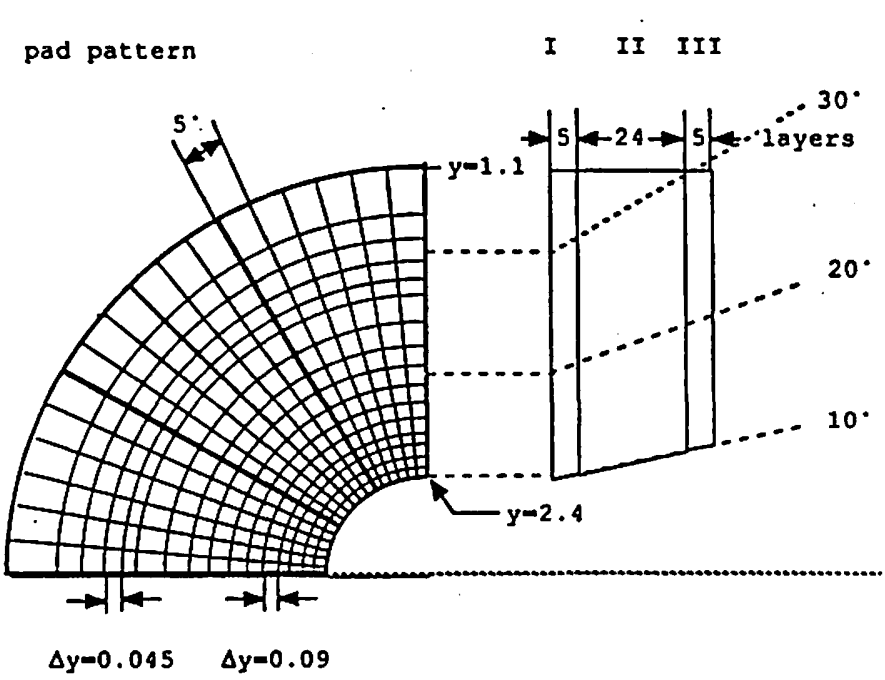

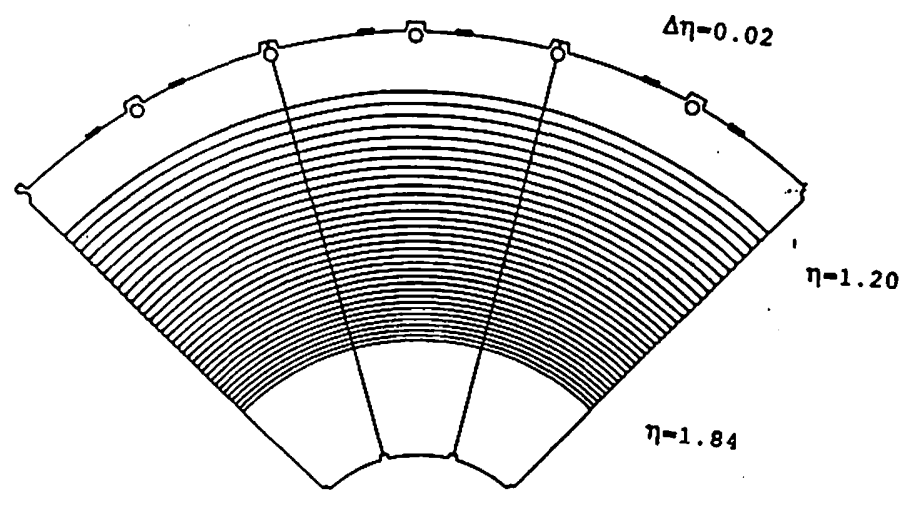

Fig.2-5-b

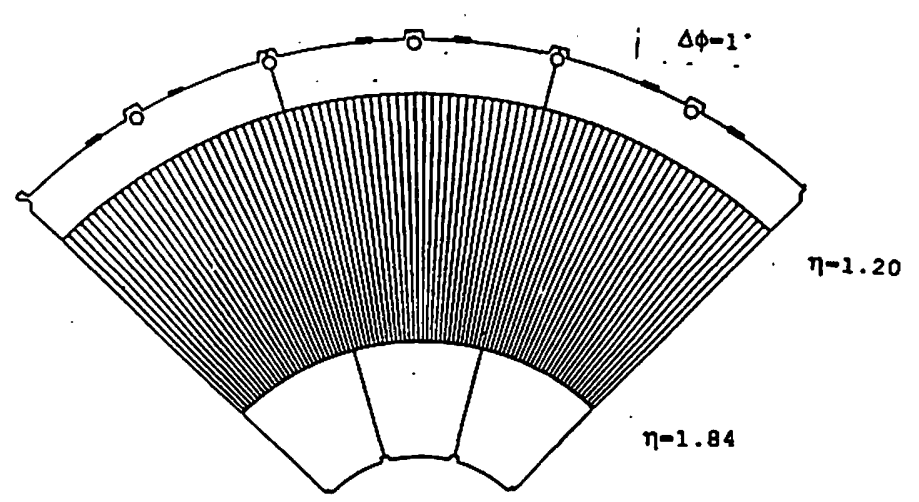




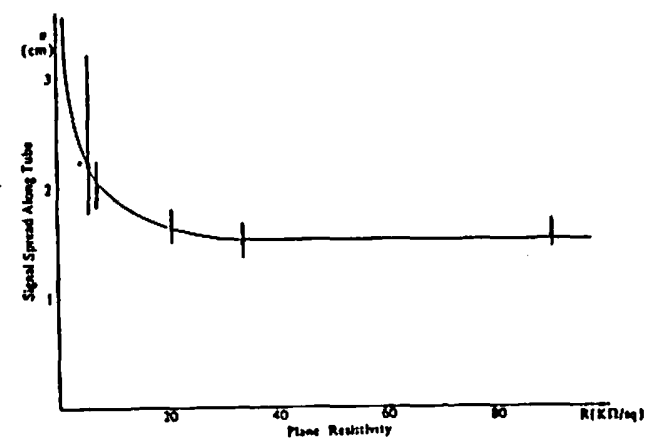

Fig. 2-6

\section{Bench Test System}

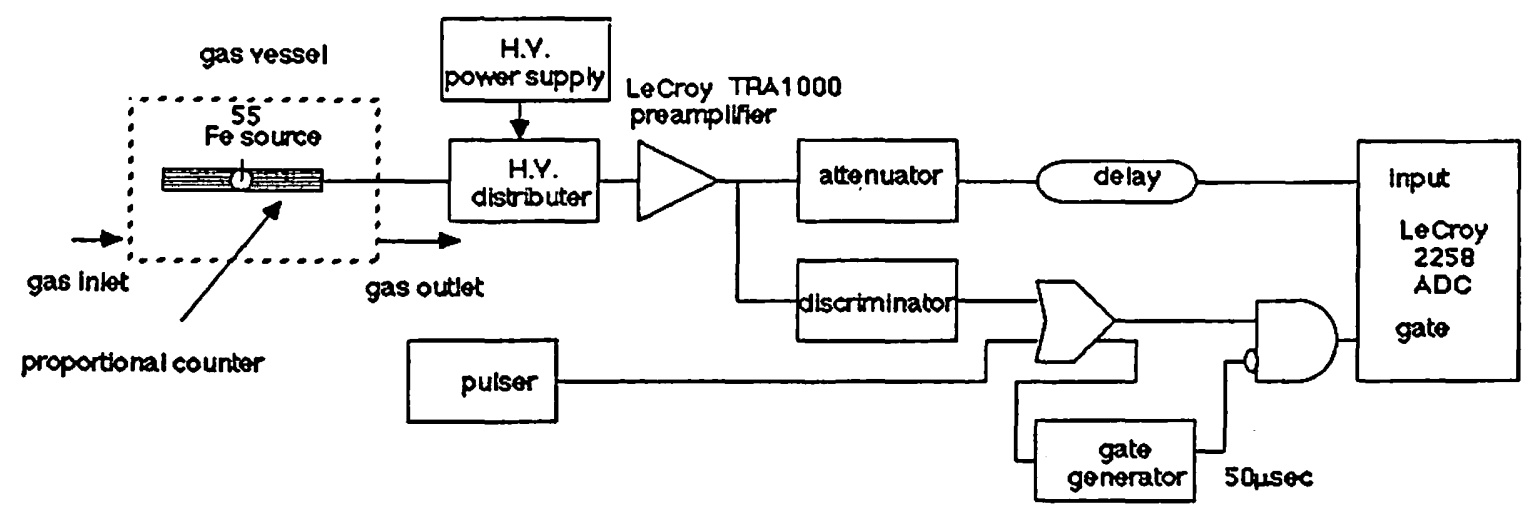

Fig. 3-1 

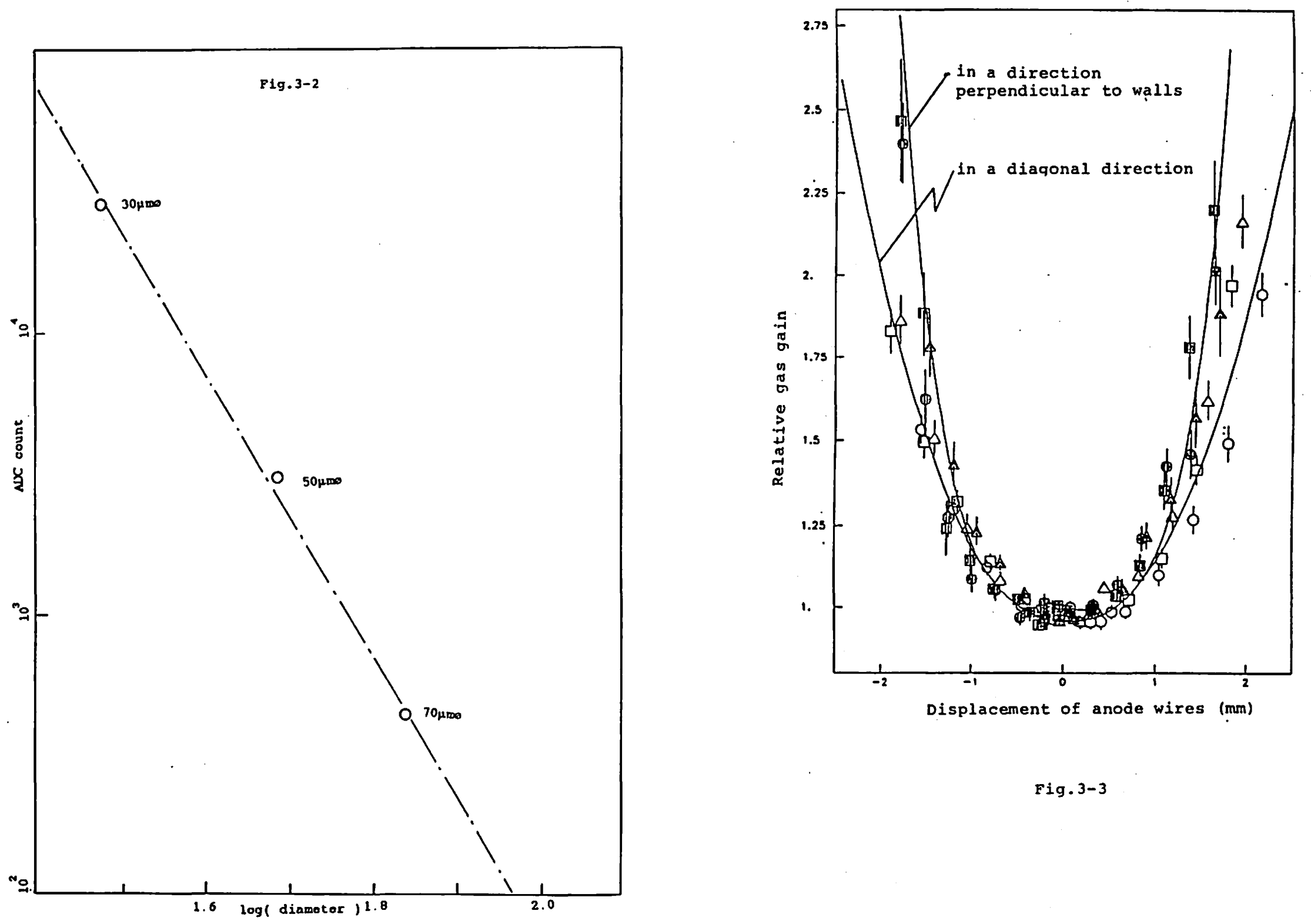

Fig. 3-3 


\section{Production process of a chamber}

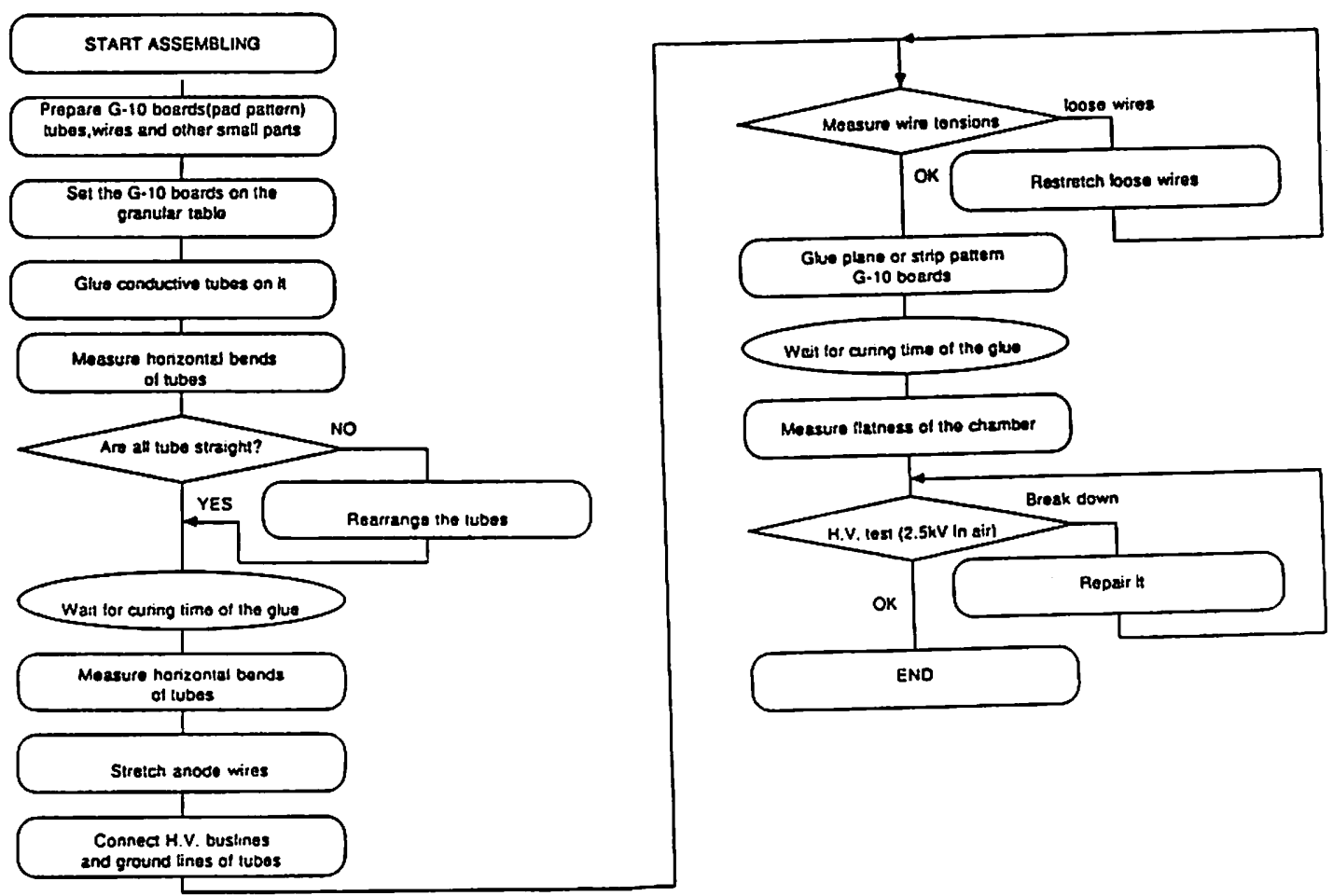

Fig. 3-4

\section{Assembling Jig of Conductive Tube Array}

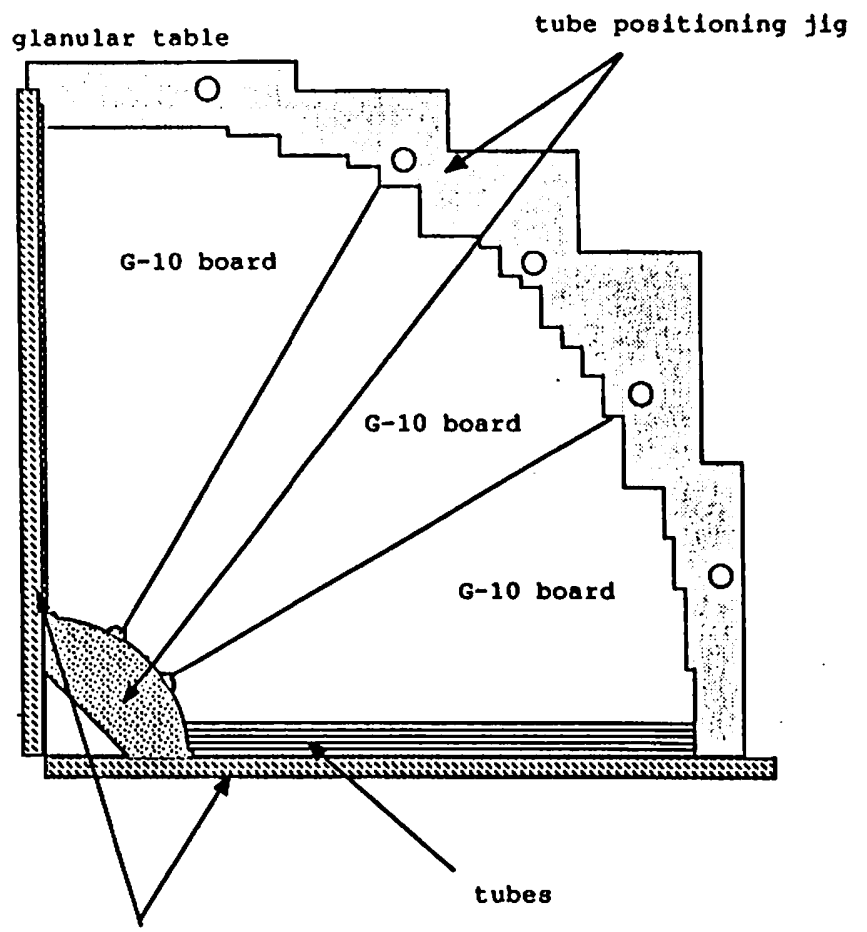

aluminlum spacer 


\section{Automatic wire tension measurement sytem}

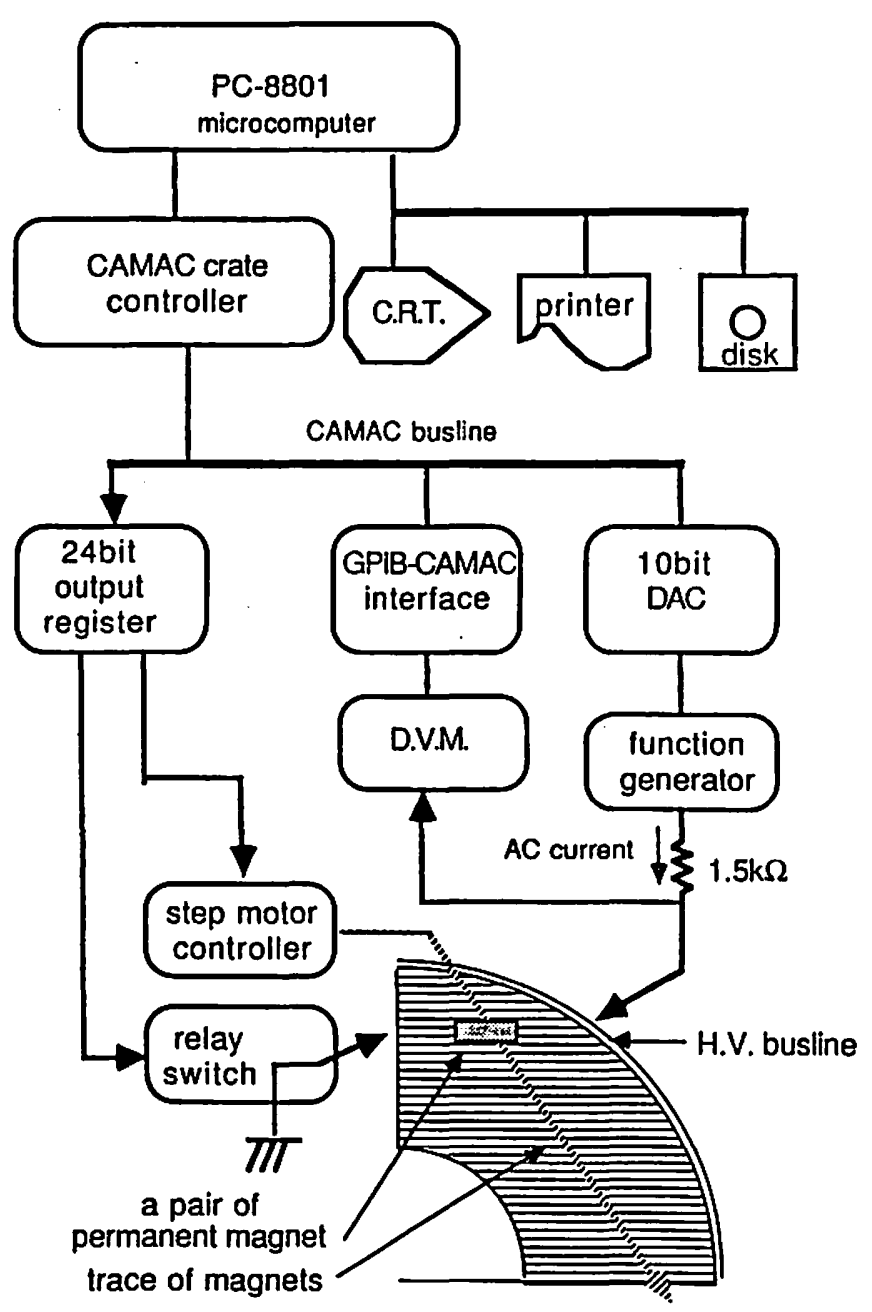

COSMIC RAY TEST SYSTEM

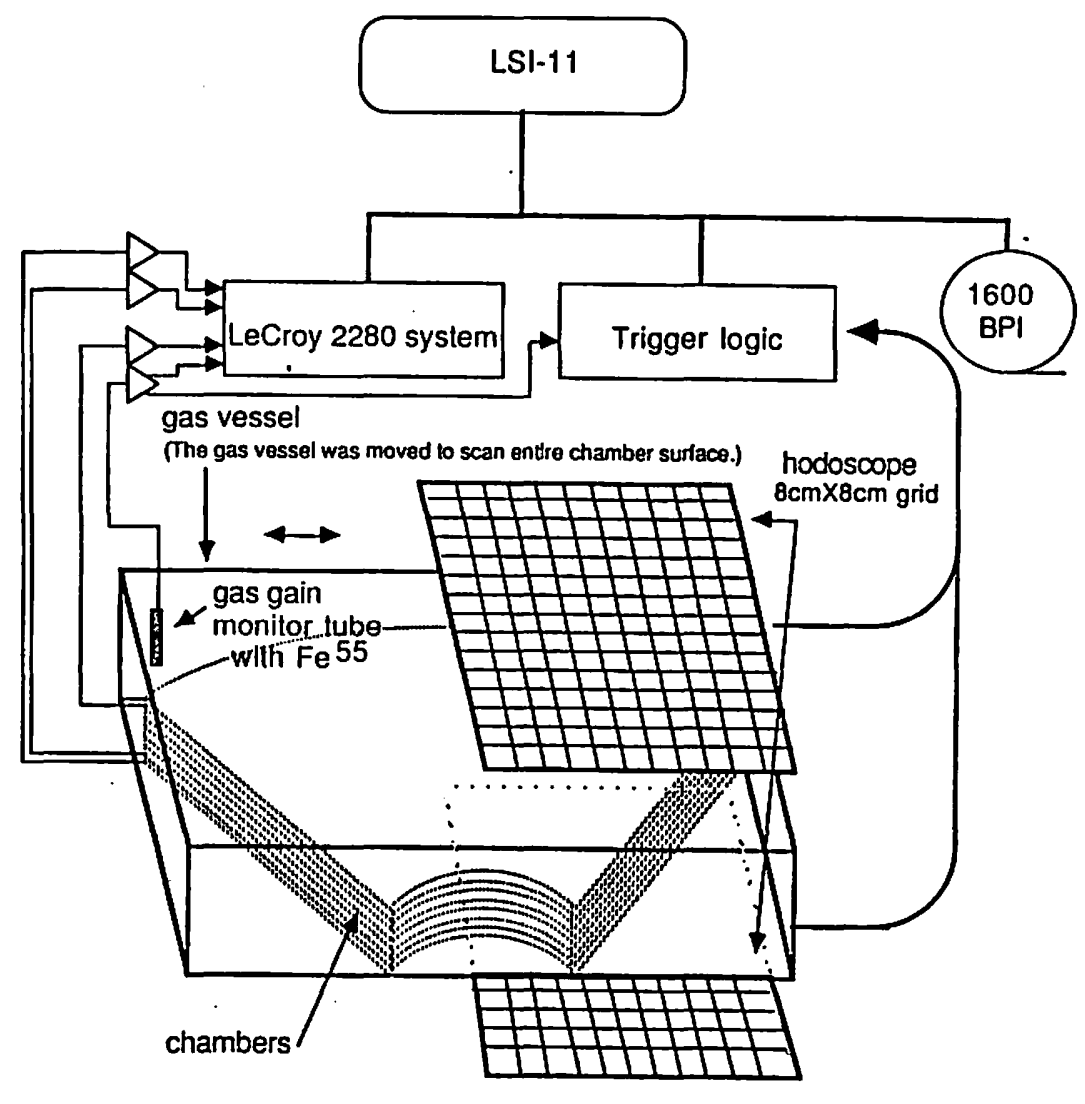

Fig. 3-7 


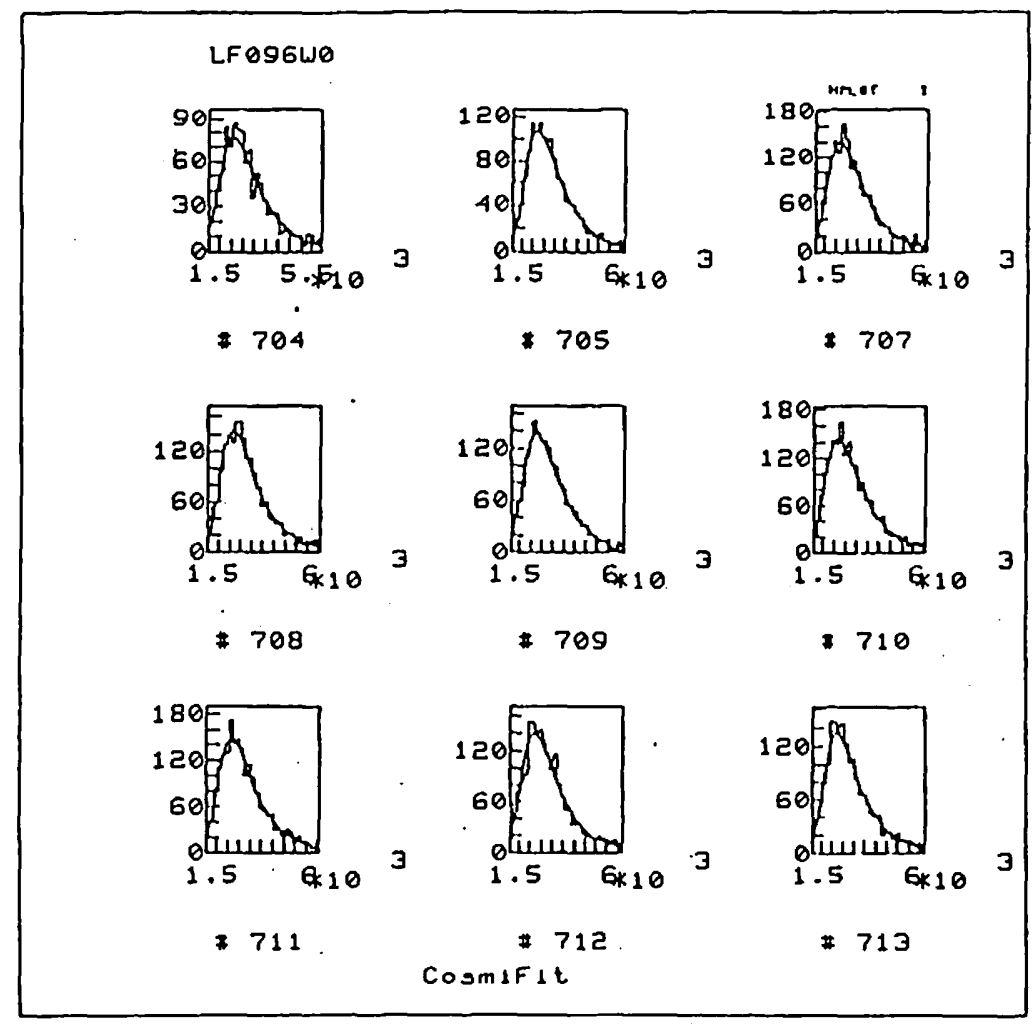

Fig. 3-8

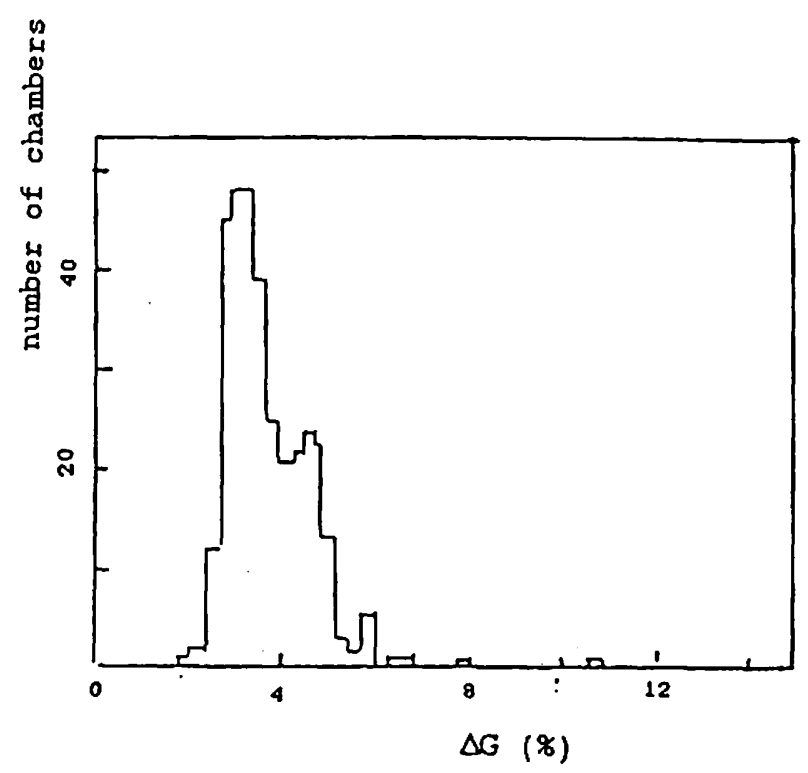

Fig.3-9. 


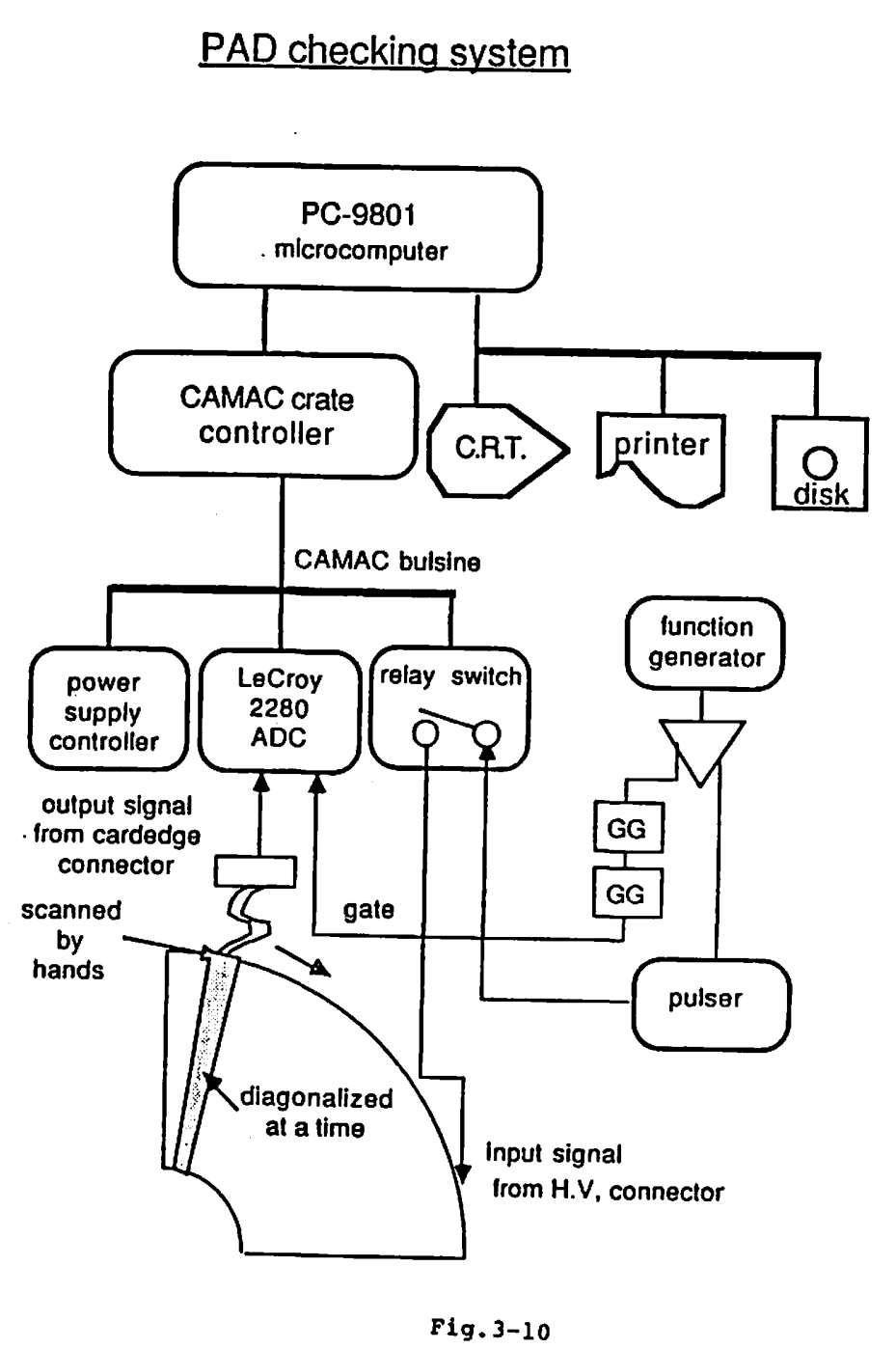
Pulse Height Observed by
Pad Checking System
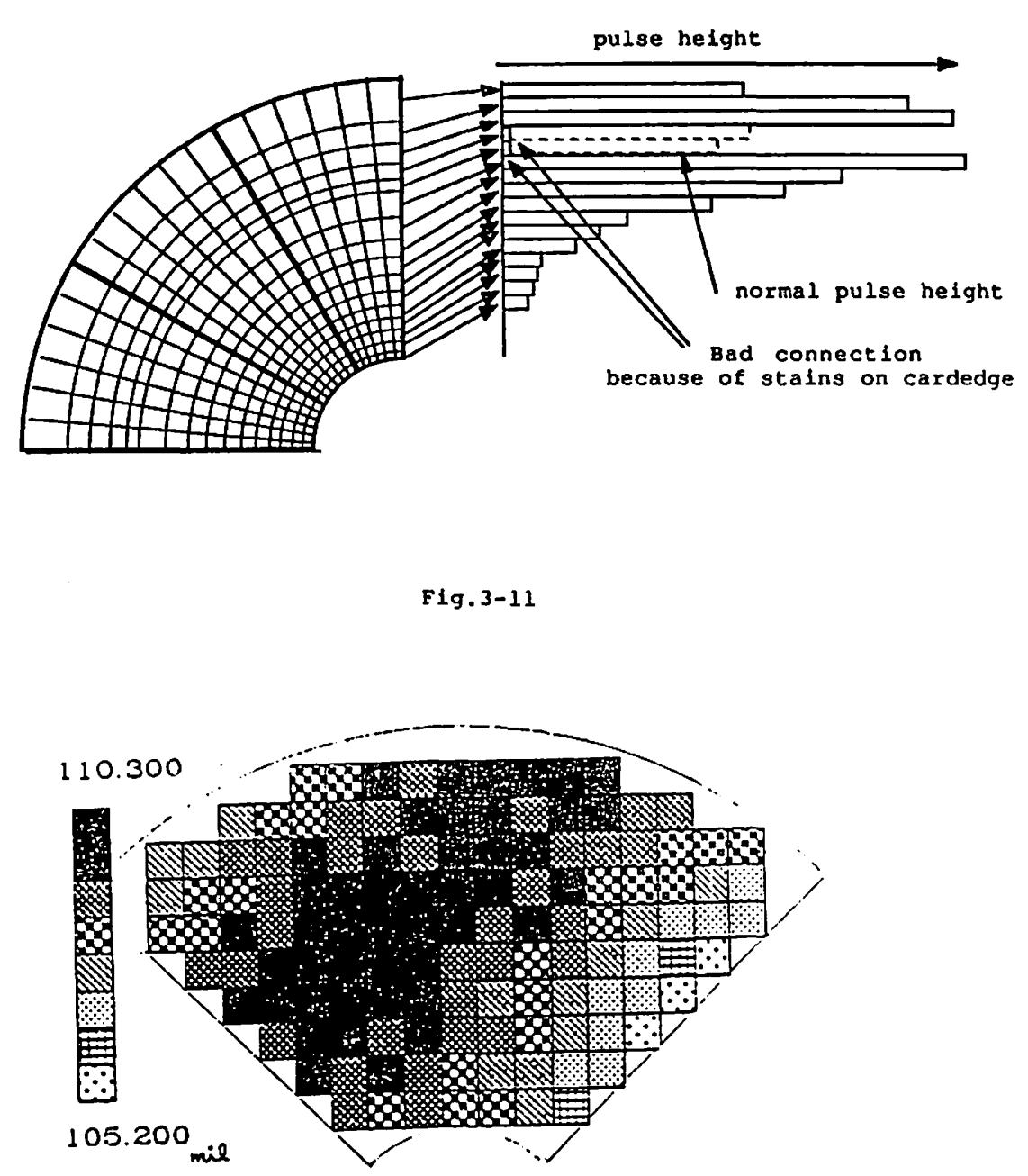

Fig. 3-12 


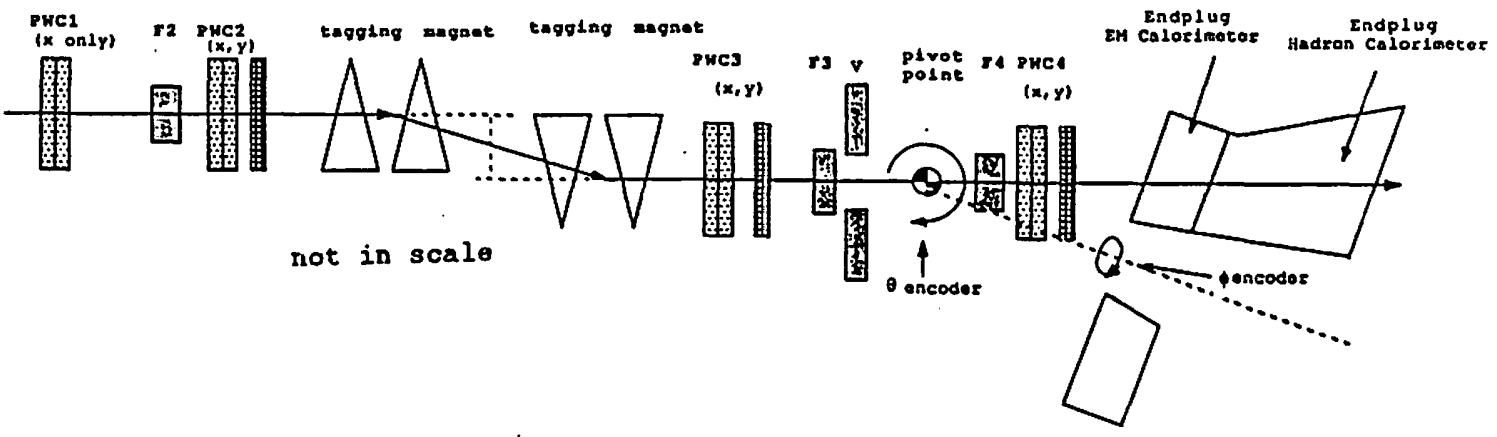

Fig.4-1

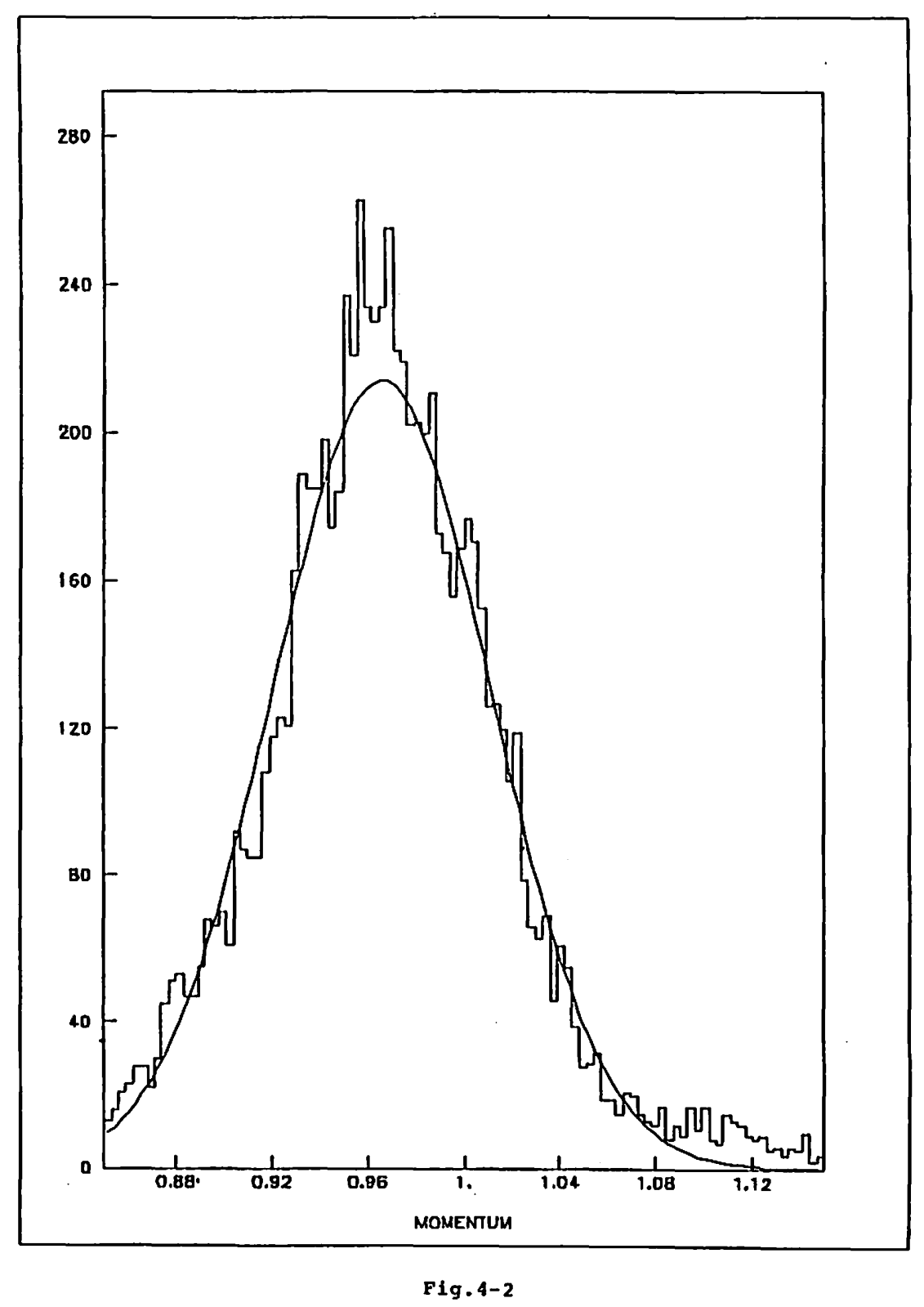




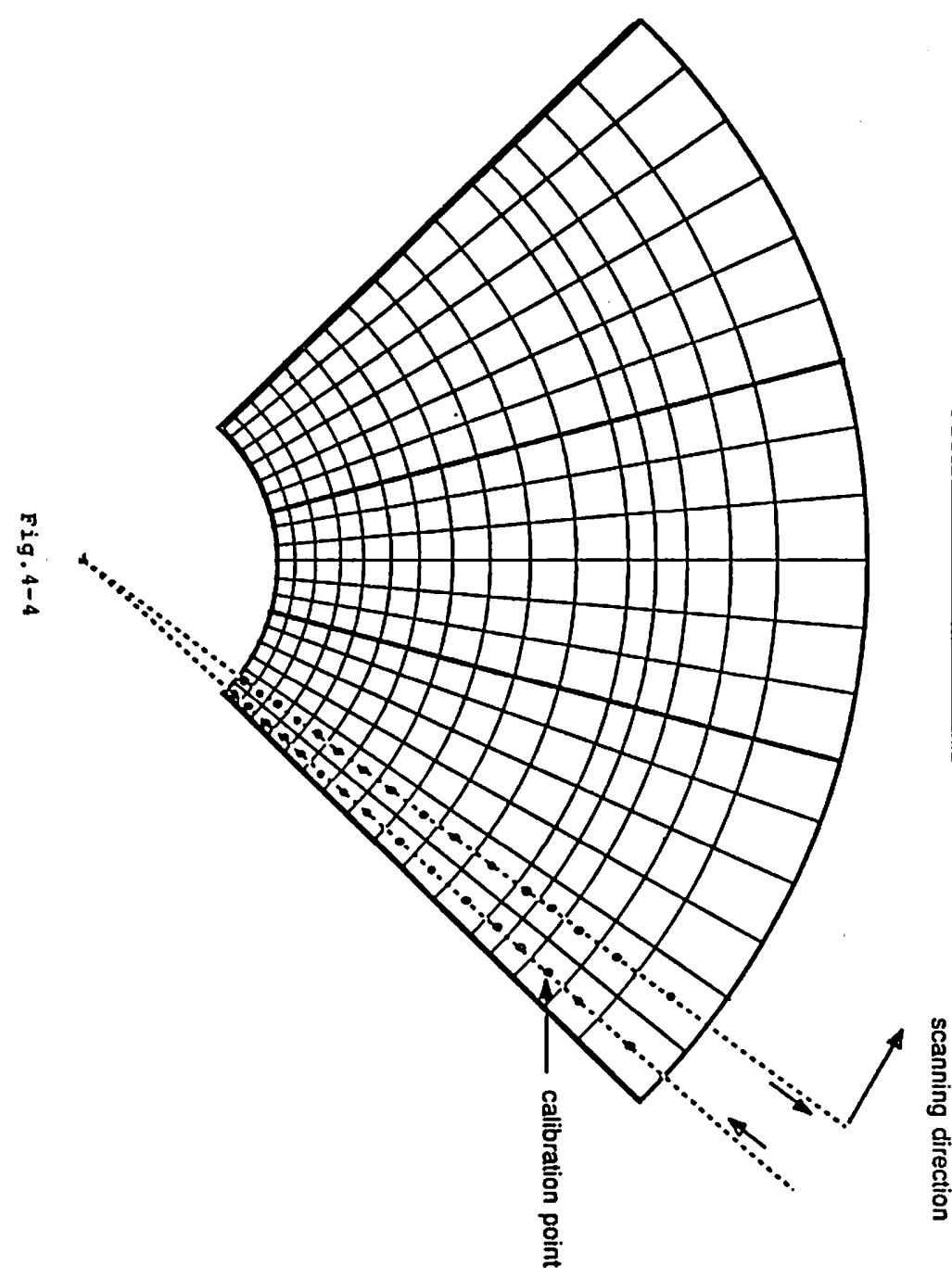

Rotating Stand Control System

סיב.
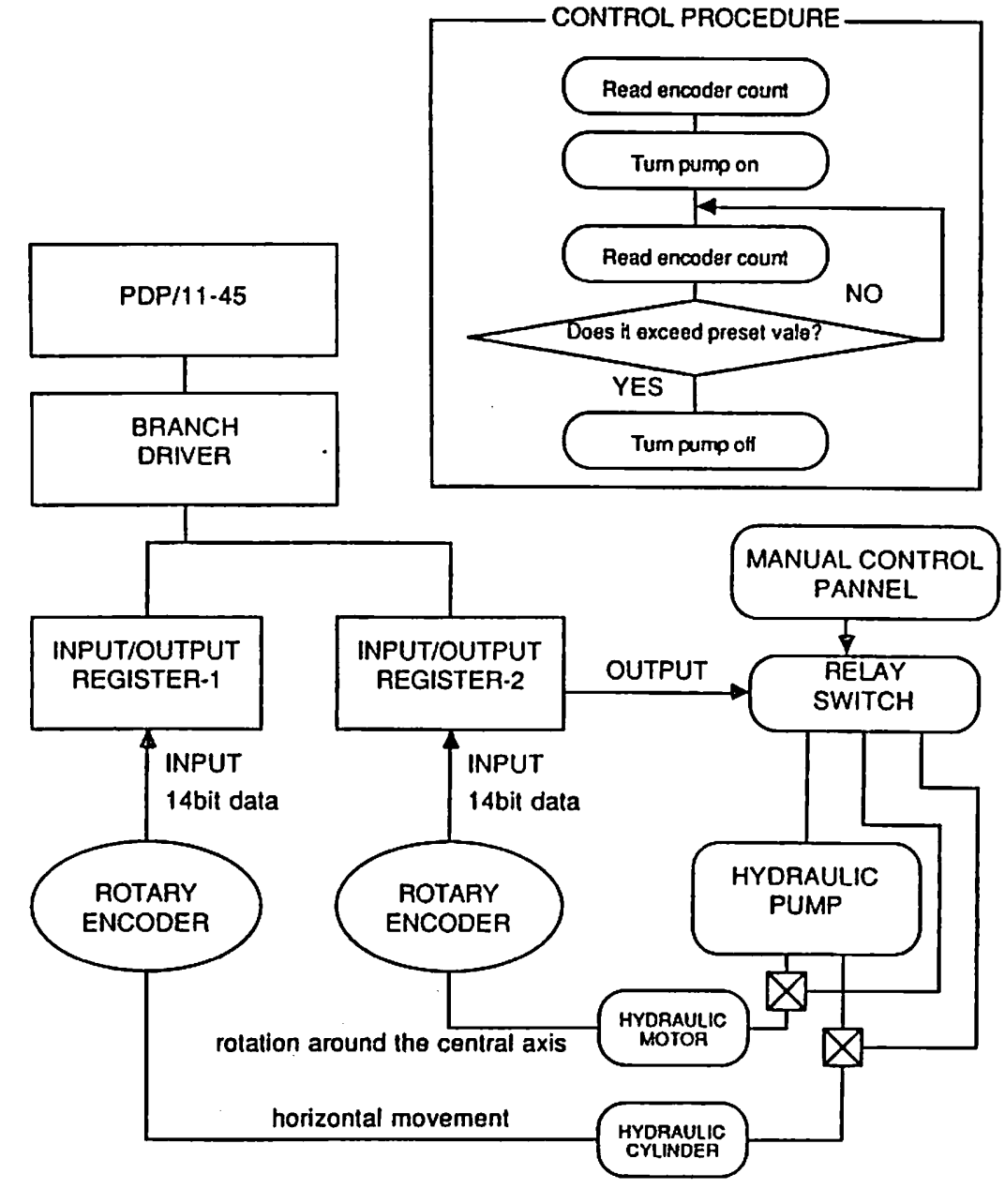

Fig.4-3 


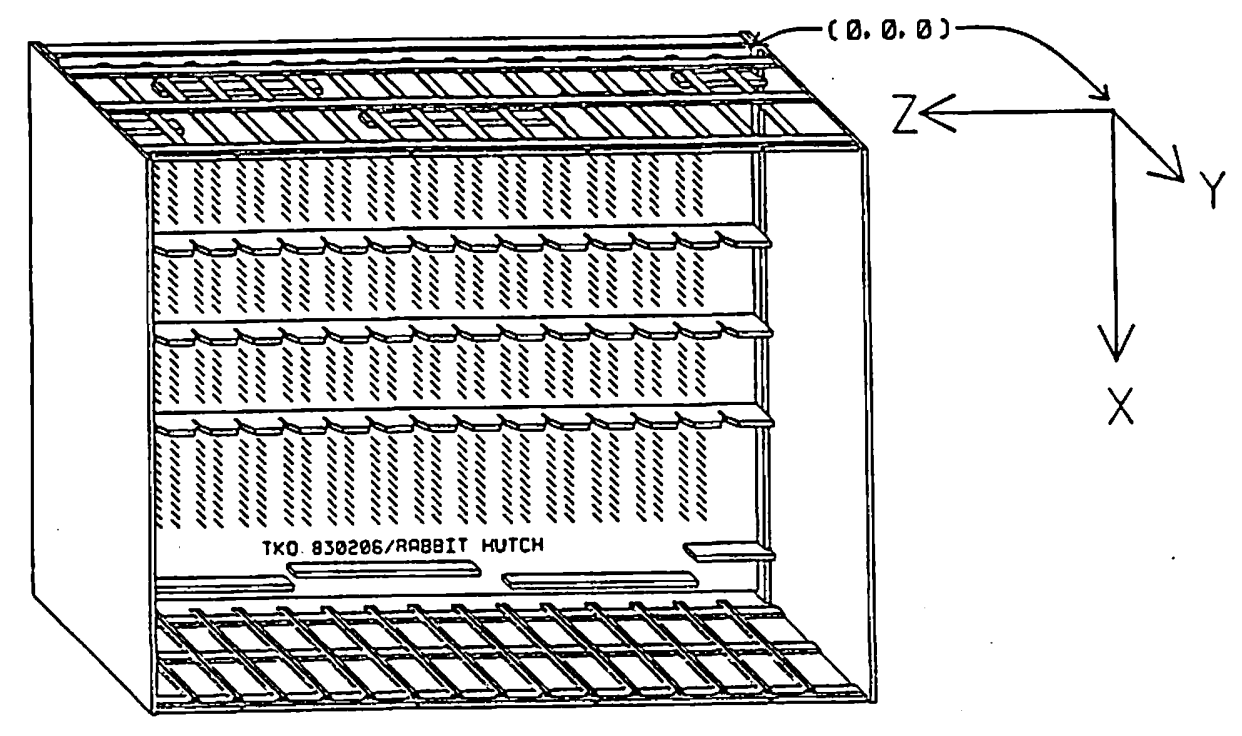

RABBIT HUTCH

Fig.4-5

\section{Configulation of Readout System at M-bottom beamline}

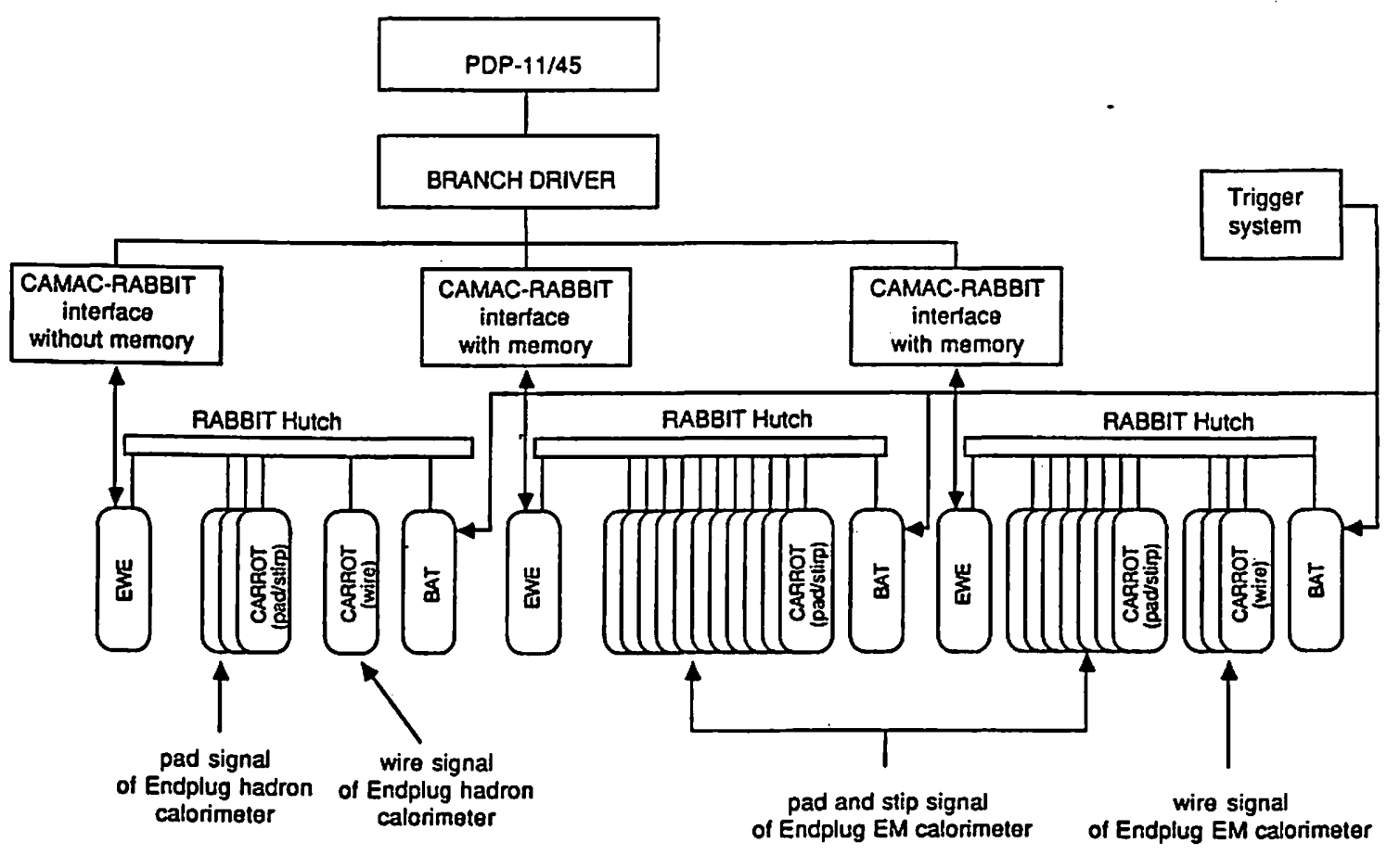

Fig. 4-6 


\section{Charge Sensitive Amplifier Module}

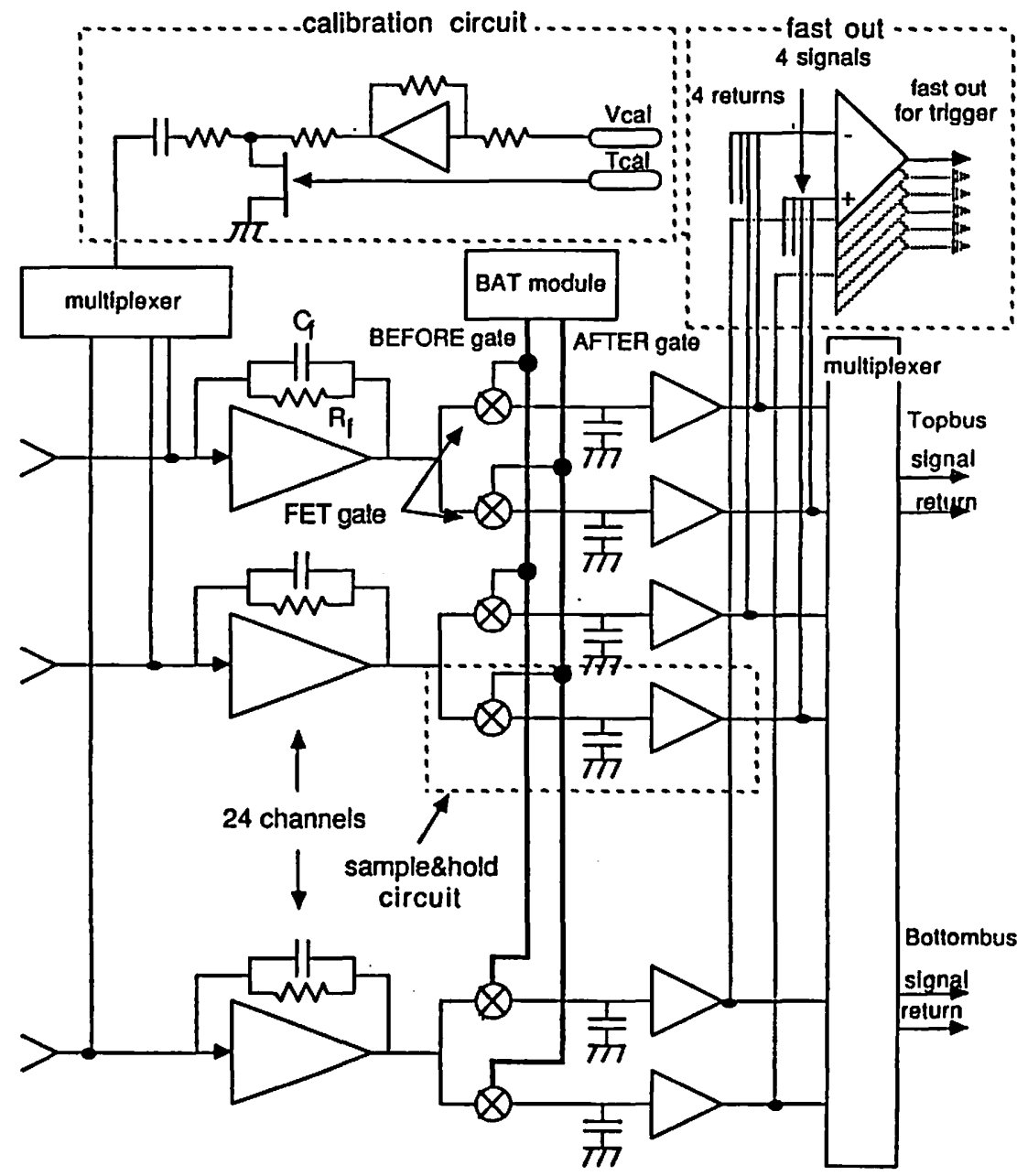

FIg.4-7

\section{Timing Chart of the sample\&hold circuint}

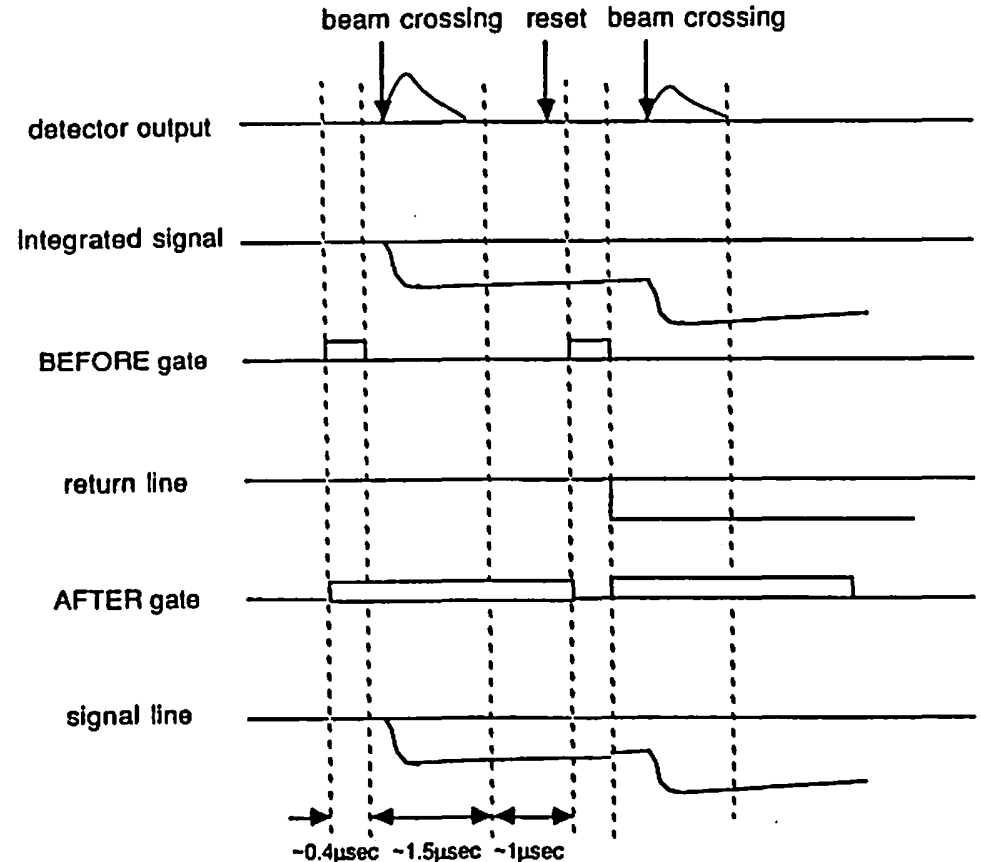

Fig. 4-8 


\section{Blockdiagram of CAMAC-RABIT interface}

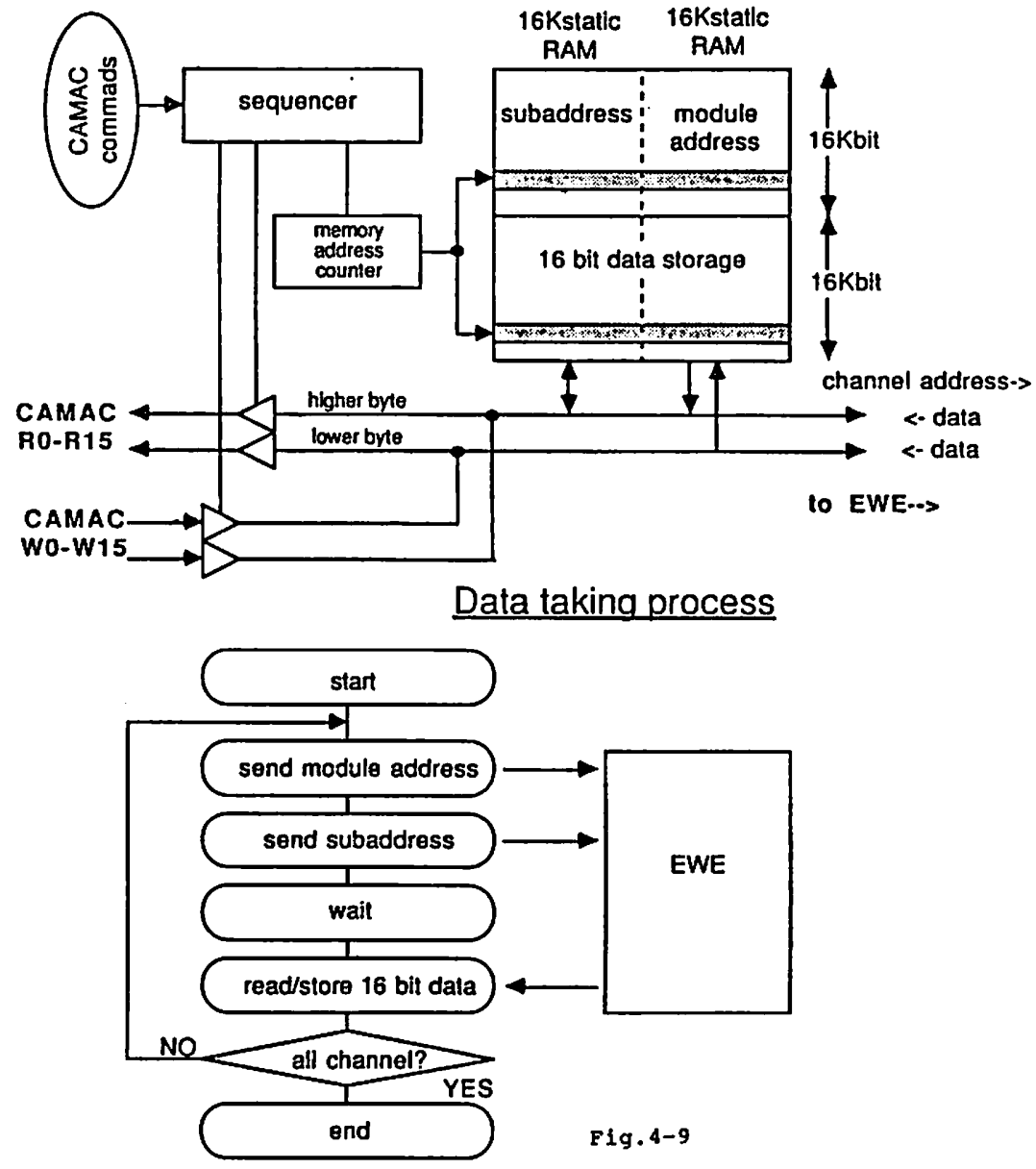

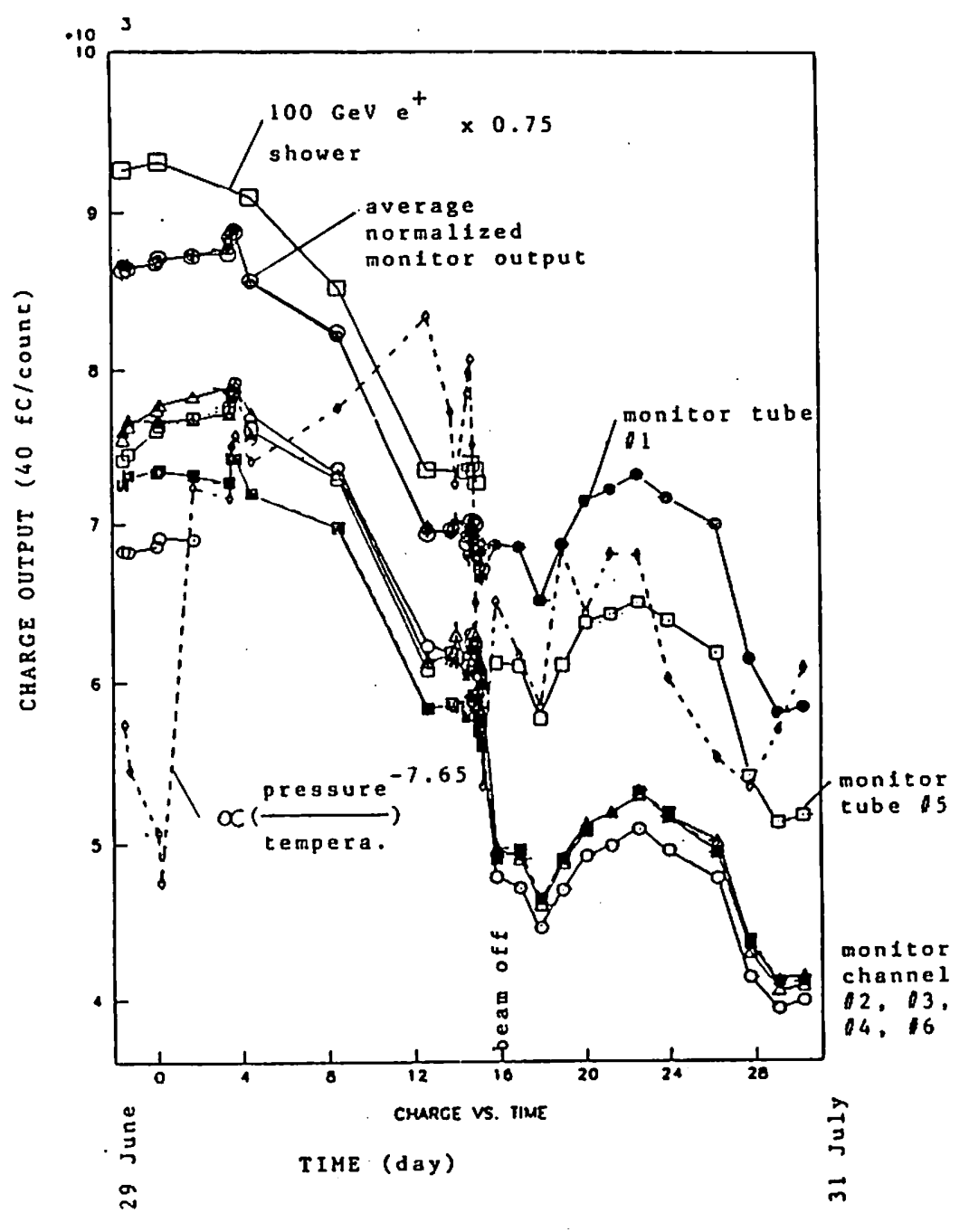

Fig. 4-10 
Pressure vs. gas gain

(constant temperature)
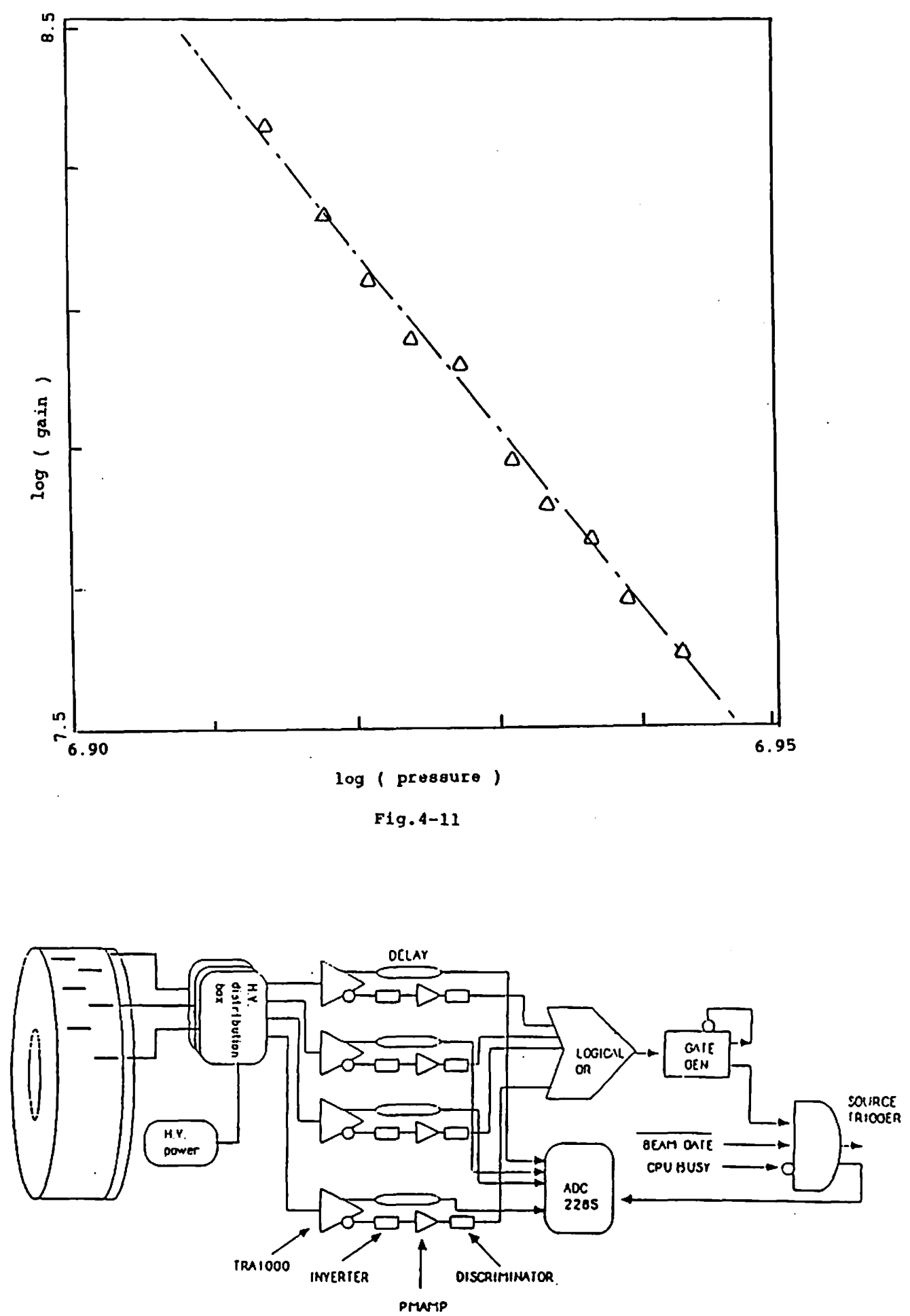

Fig.4-12 


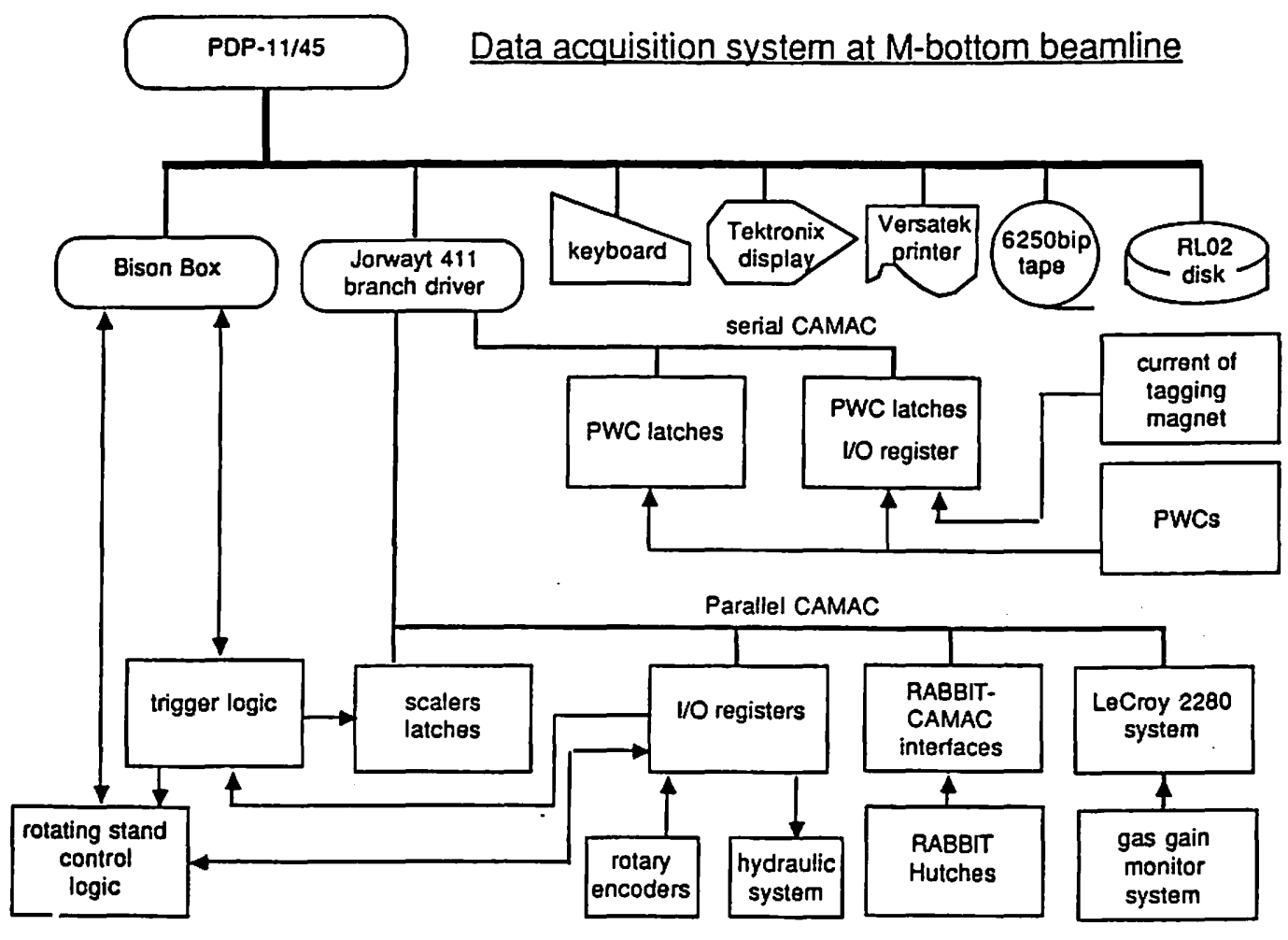

Fig. 4-13

Irigger system at M-bottom beam line
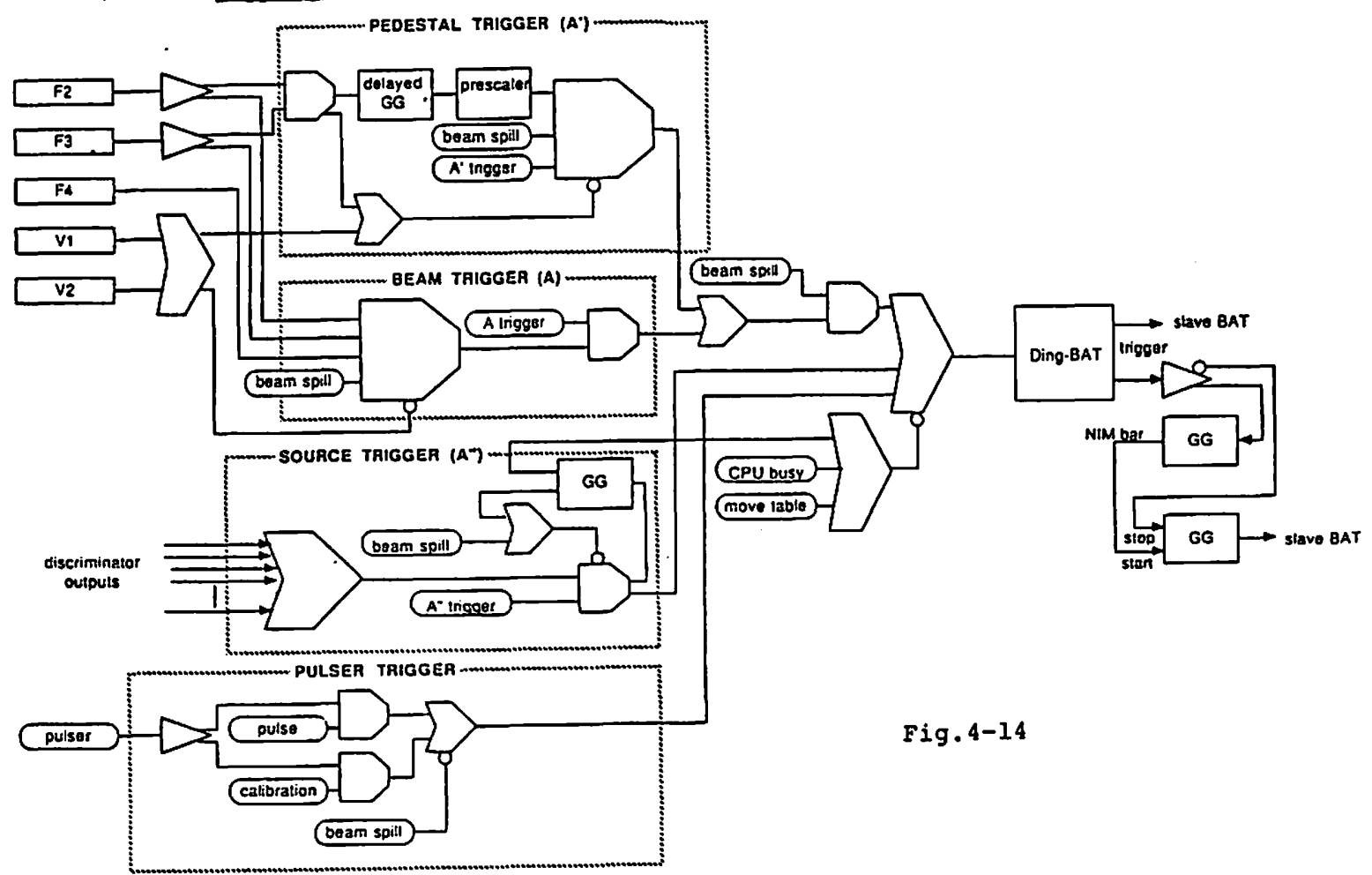

Fig.4-14 


\section{Cable connection checking process}
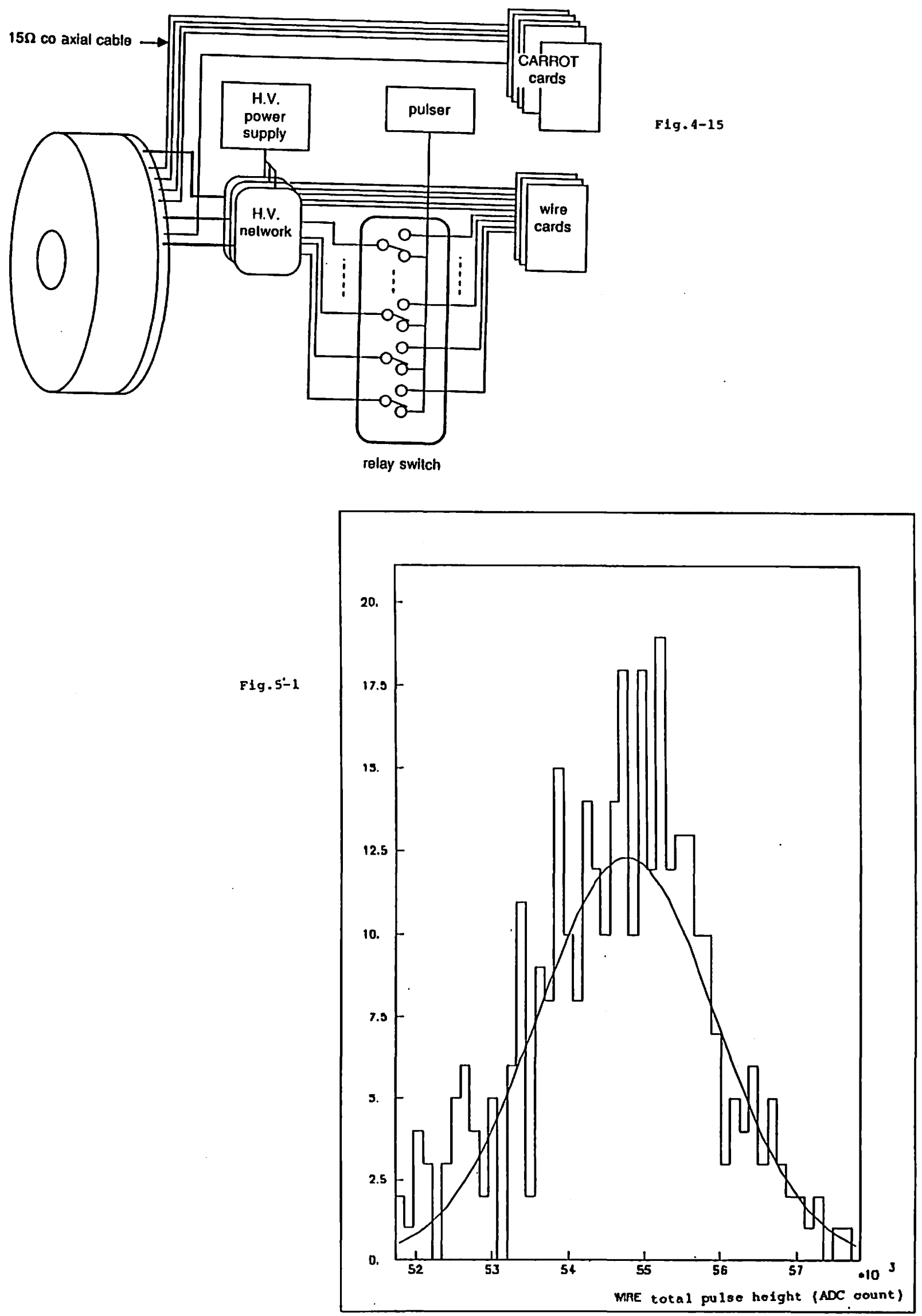
Eleotron energy ro. energy resolution

$0 \quad 1.85 \mathrm{kV}\left\{\begin{array}{l}\text { wire }(21.6 \pm 0.5) \% / \sqrt{E} \\ \text { pad }(22.8 \pm 0.6) \% / \sqrt{\mathrm{E}}\end{array}\right.$

$\square \quad 1.80 \mathrm{kV}\left\{\begin{array}{l}\text { rire }(22.3 \pm 0.4) \% / \sqrt{E} \\ \text { pad }(23.3 \pm 0.5) \% / \sqrt{E}\end{array}\right.$

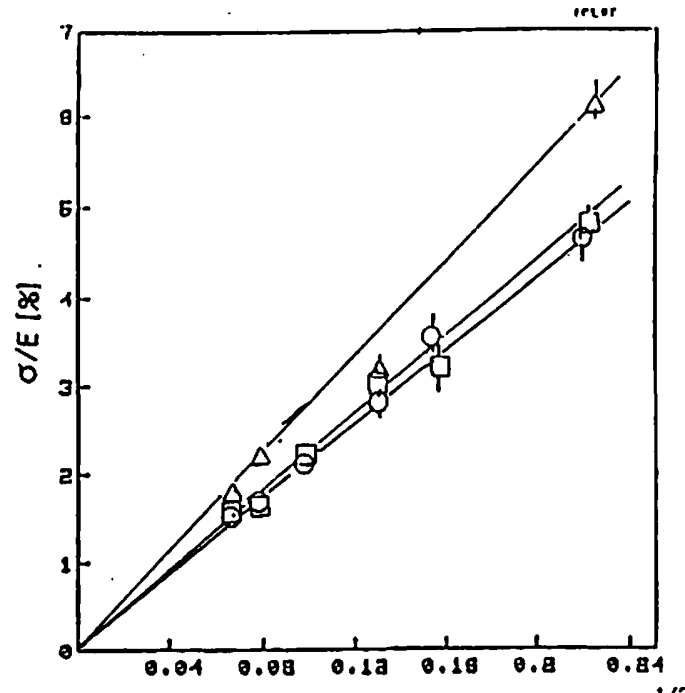

EHEROY US. ENEROY REgOLUTION $1 / \sqrt{E} \mathrm{GeV}^{-1 / 2}$

(WIRE)

Eloctron energy vo. total charge

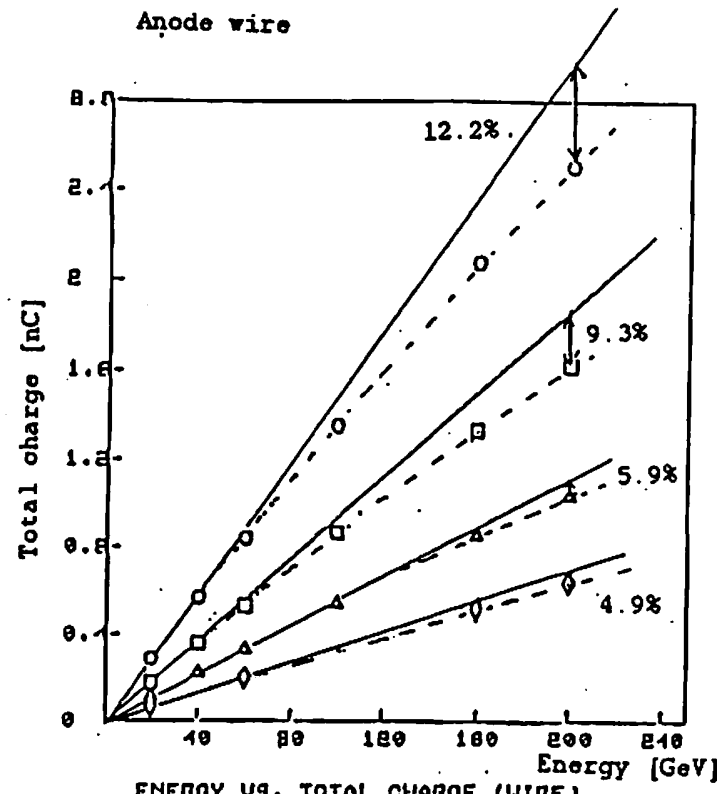

EMEROY Ug, TOTAL CHIAROE (WSRE
$\Delta \quad 1.75 \mathrm{kV}\left\{\begin{array}{l}\text { rire }(26.6 \pm 0.6) \% / \sqrt{ } \mathrm{E} \\ \text { pad }(28.4 \pm 0.7) \% / \sqrt{\mathrm{V}}\end{array}\right.$

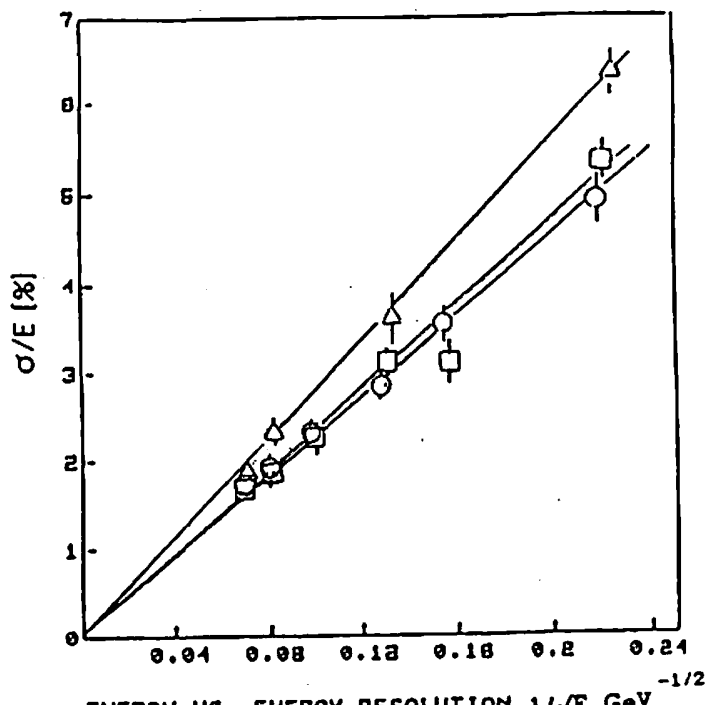

(PAD)

Fig.5-2

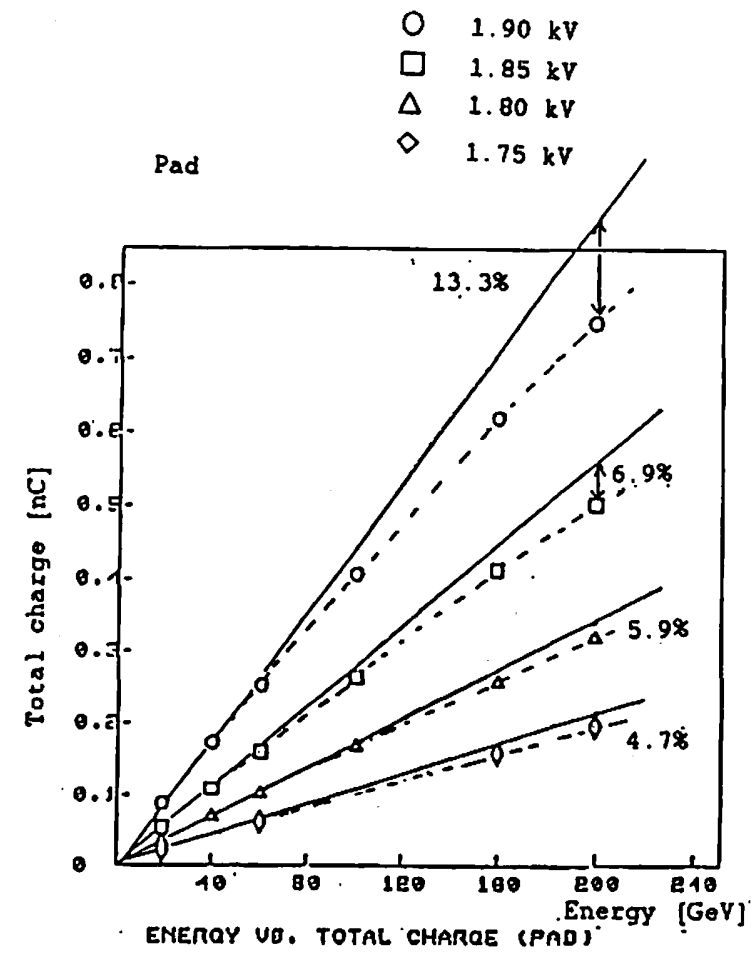

Fig. 5-4 

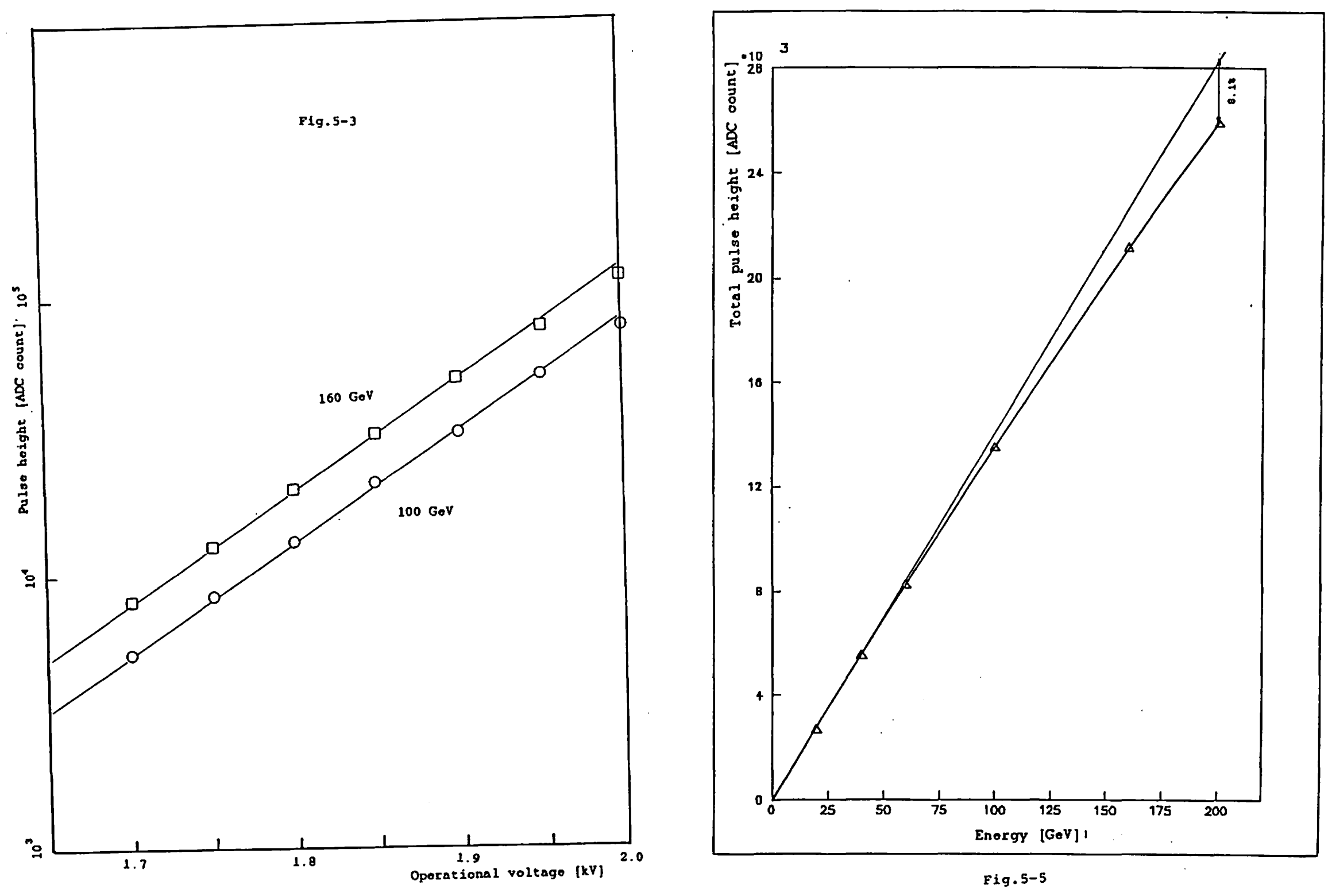

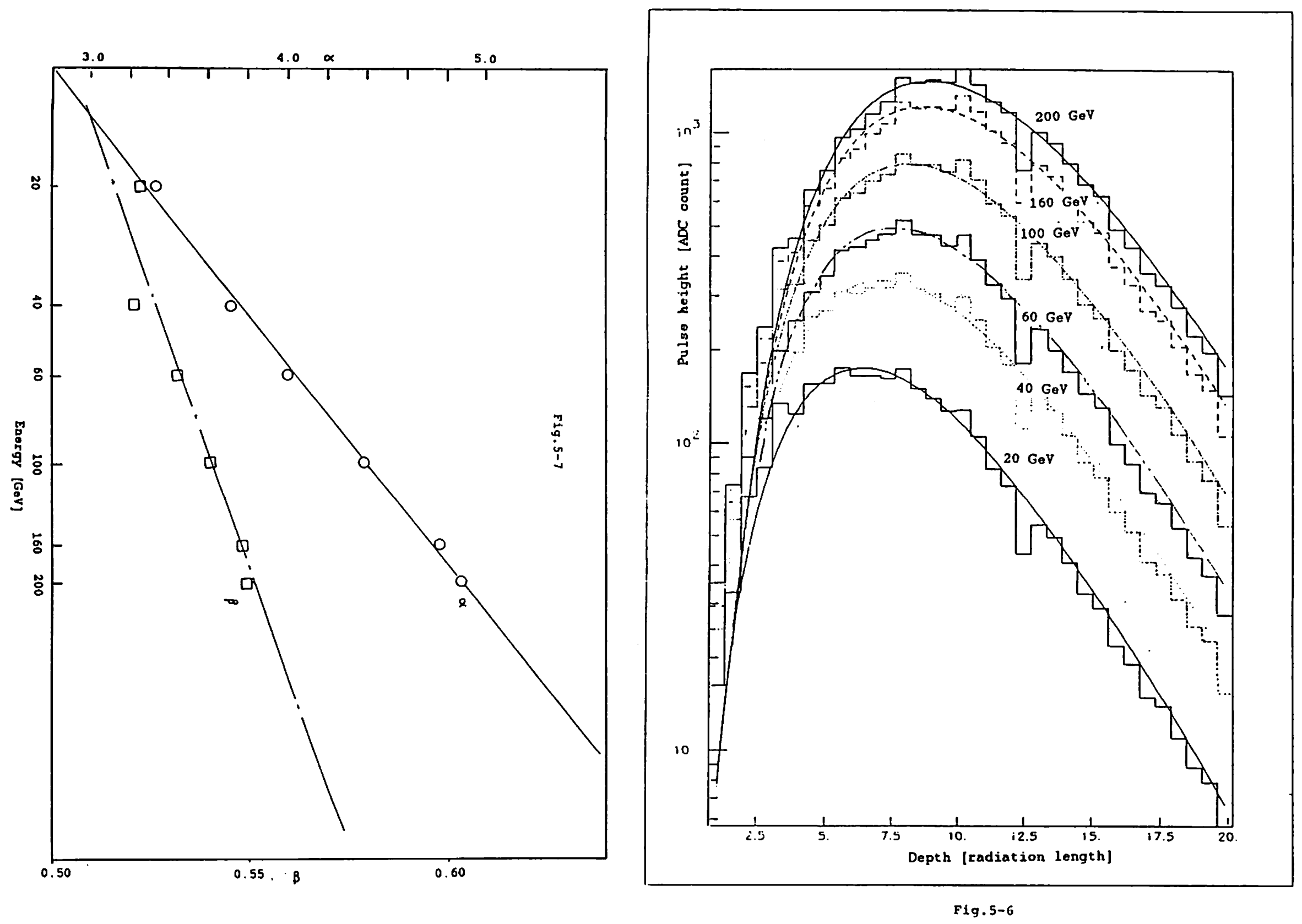


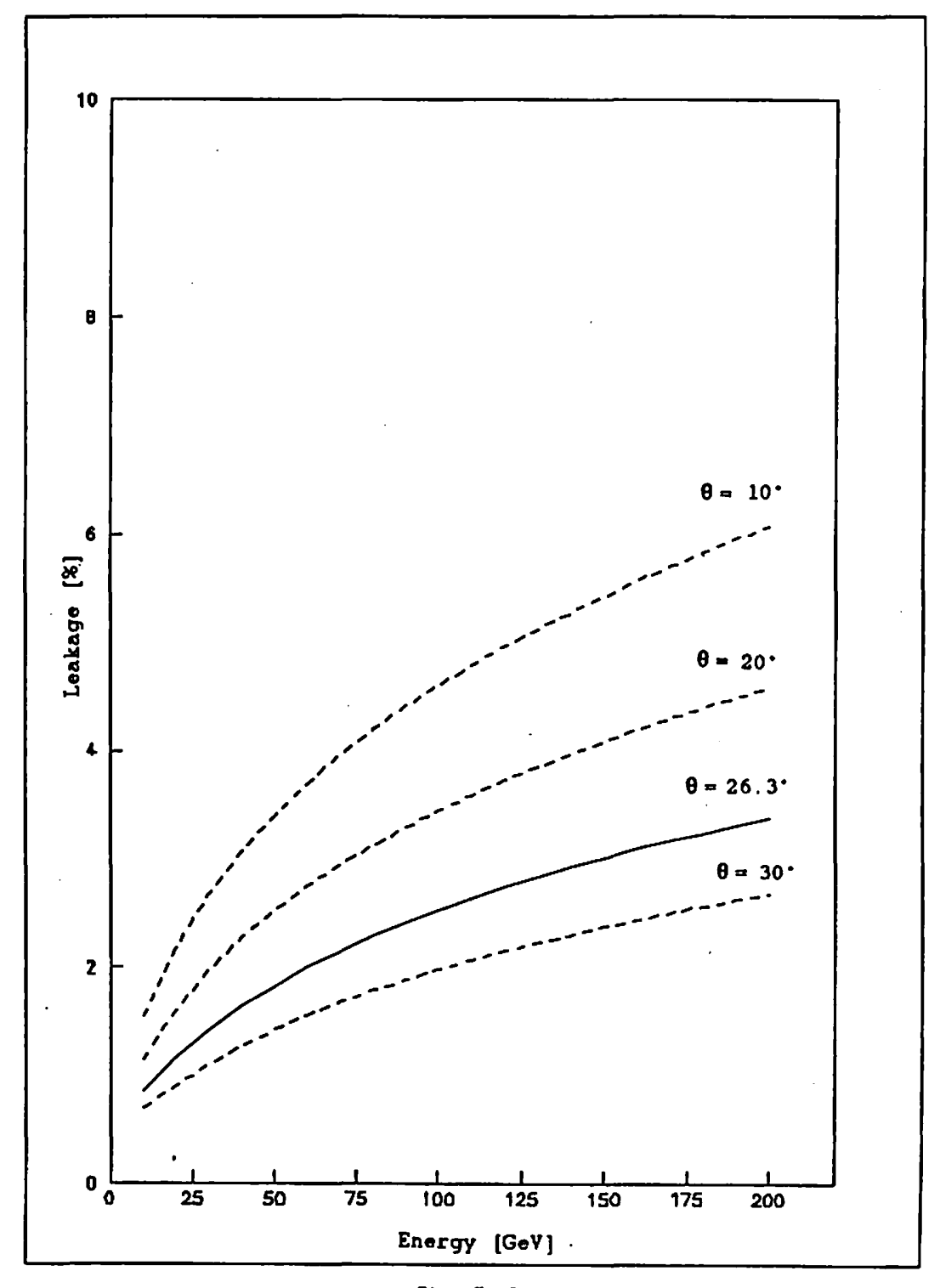

E!g. 5- 8

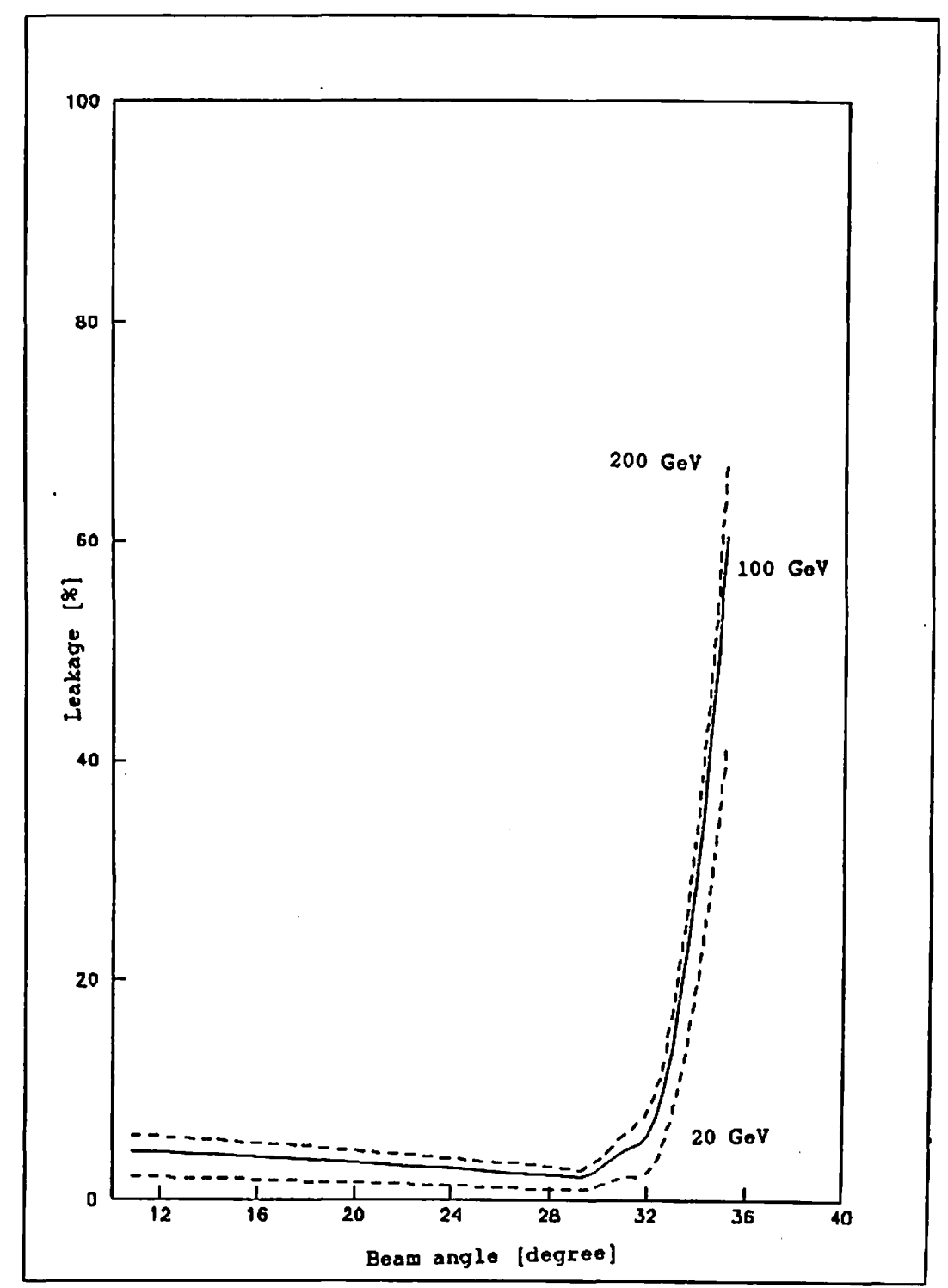

Fig. 5-9-a 

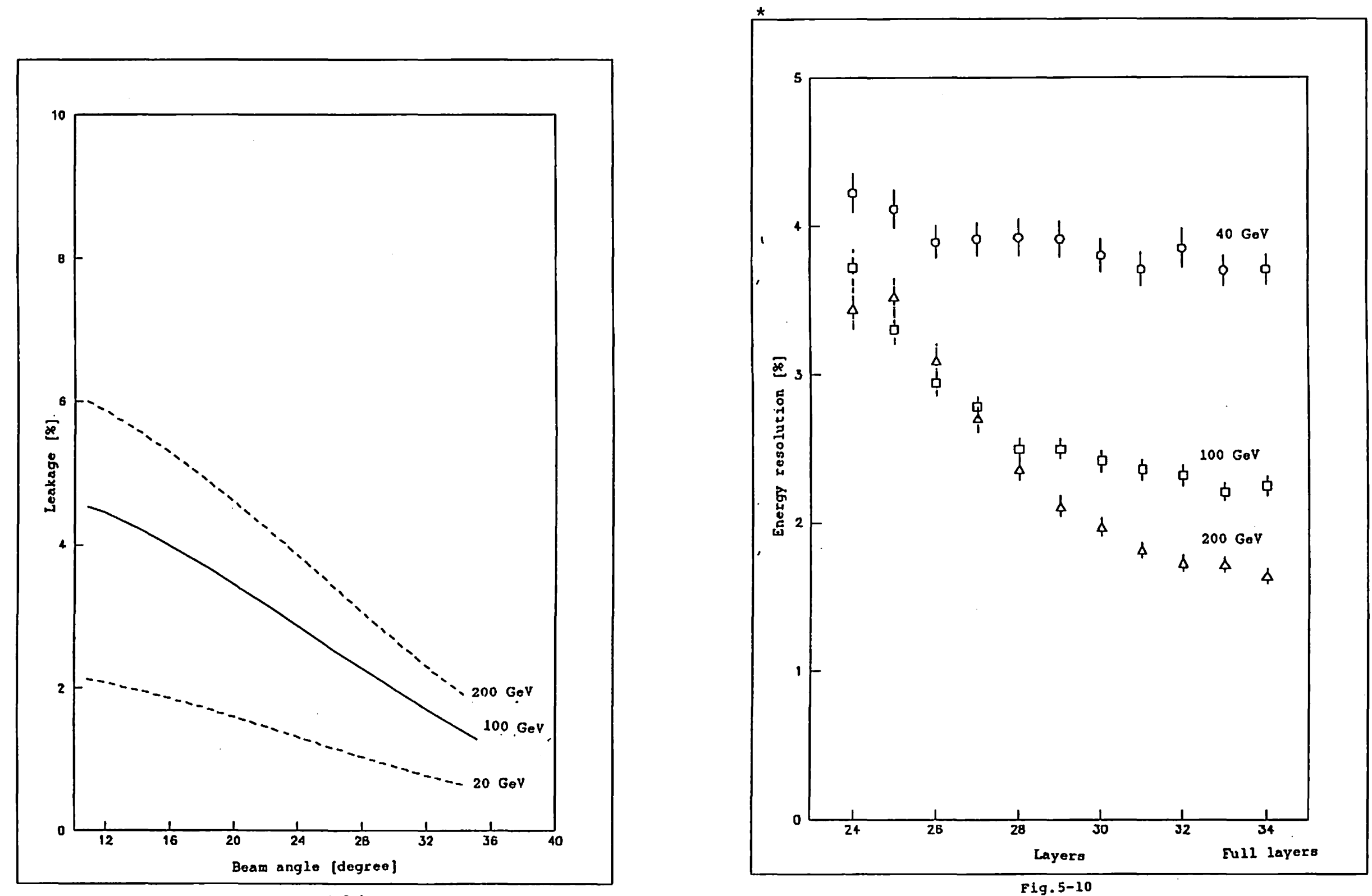

Fig. 5-9-b 

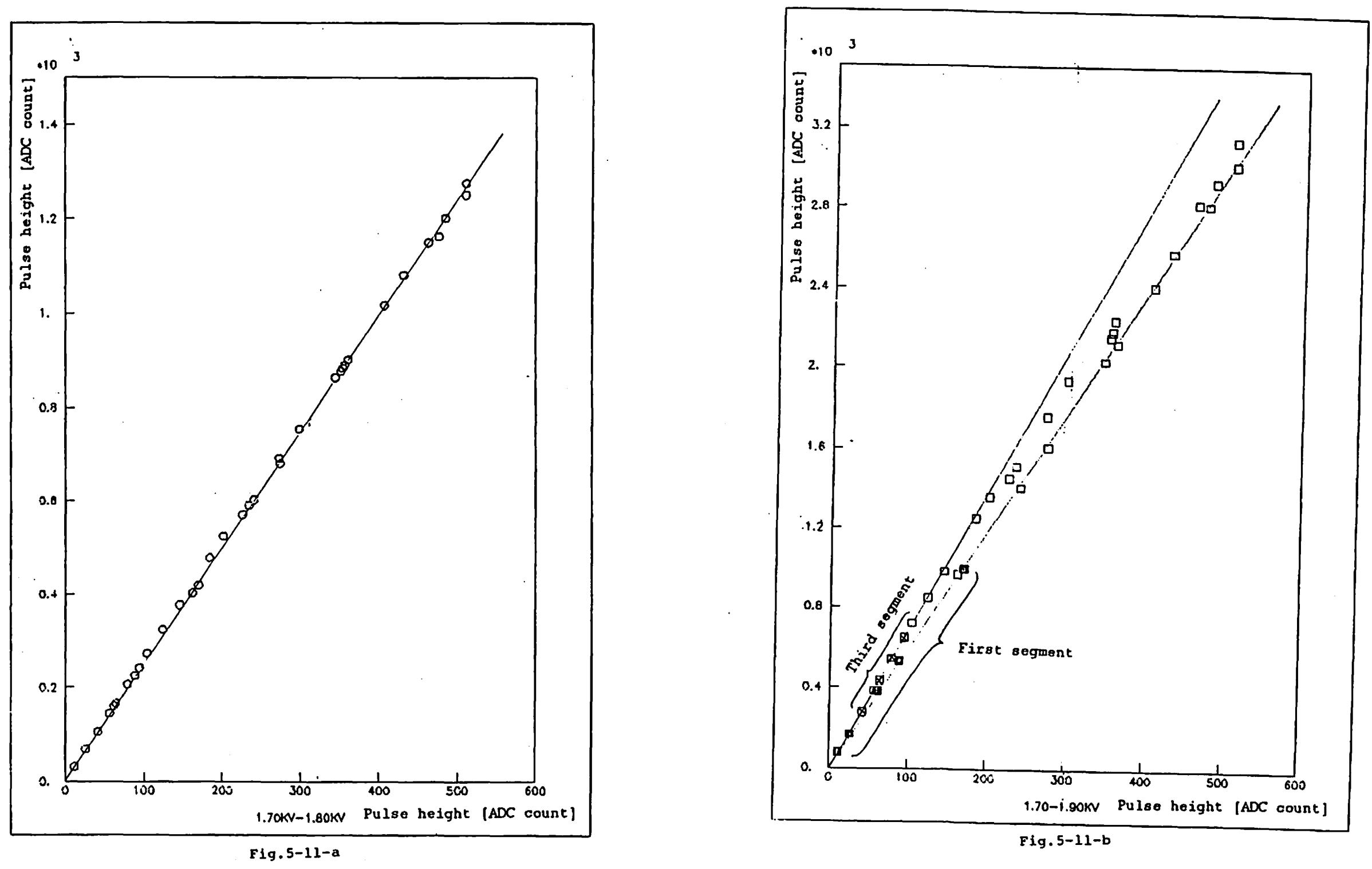


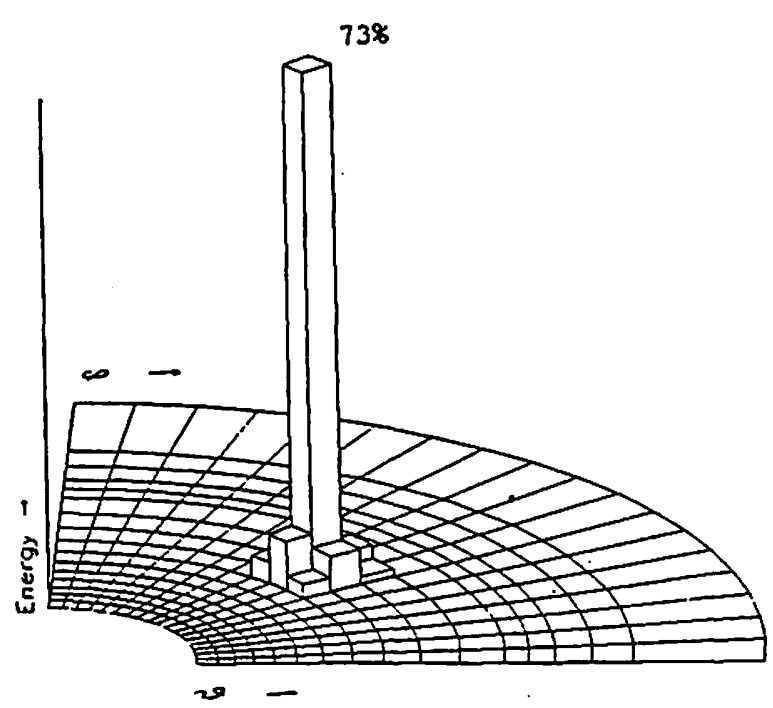

Fig.5-12

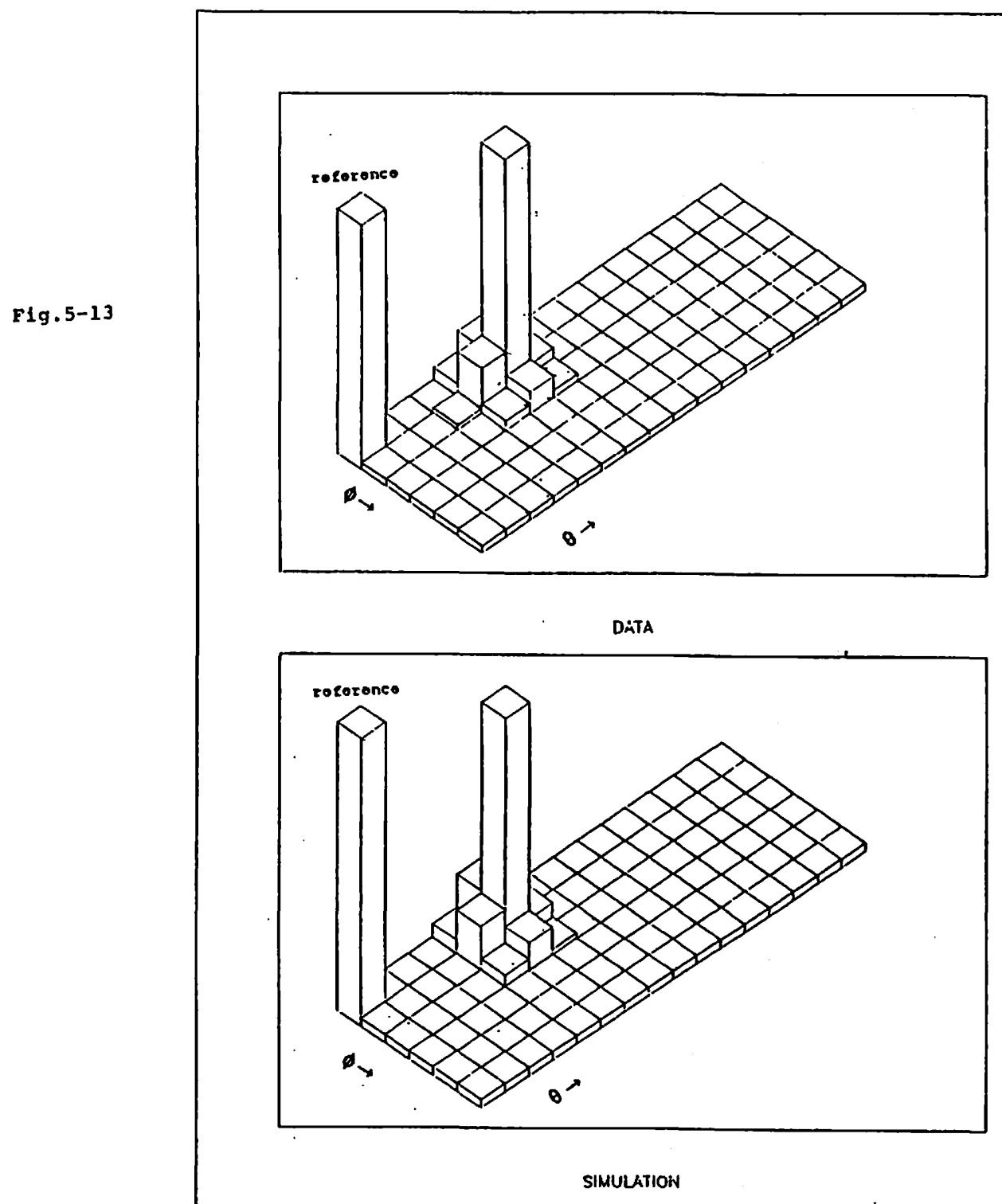



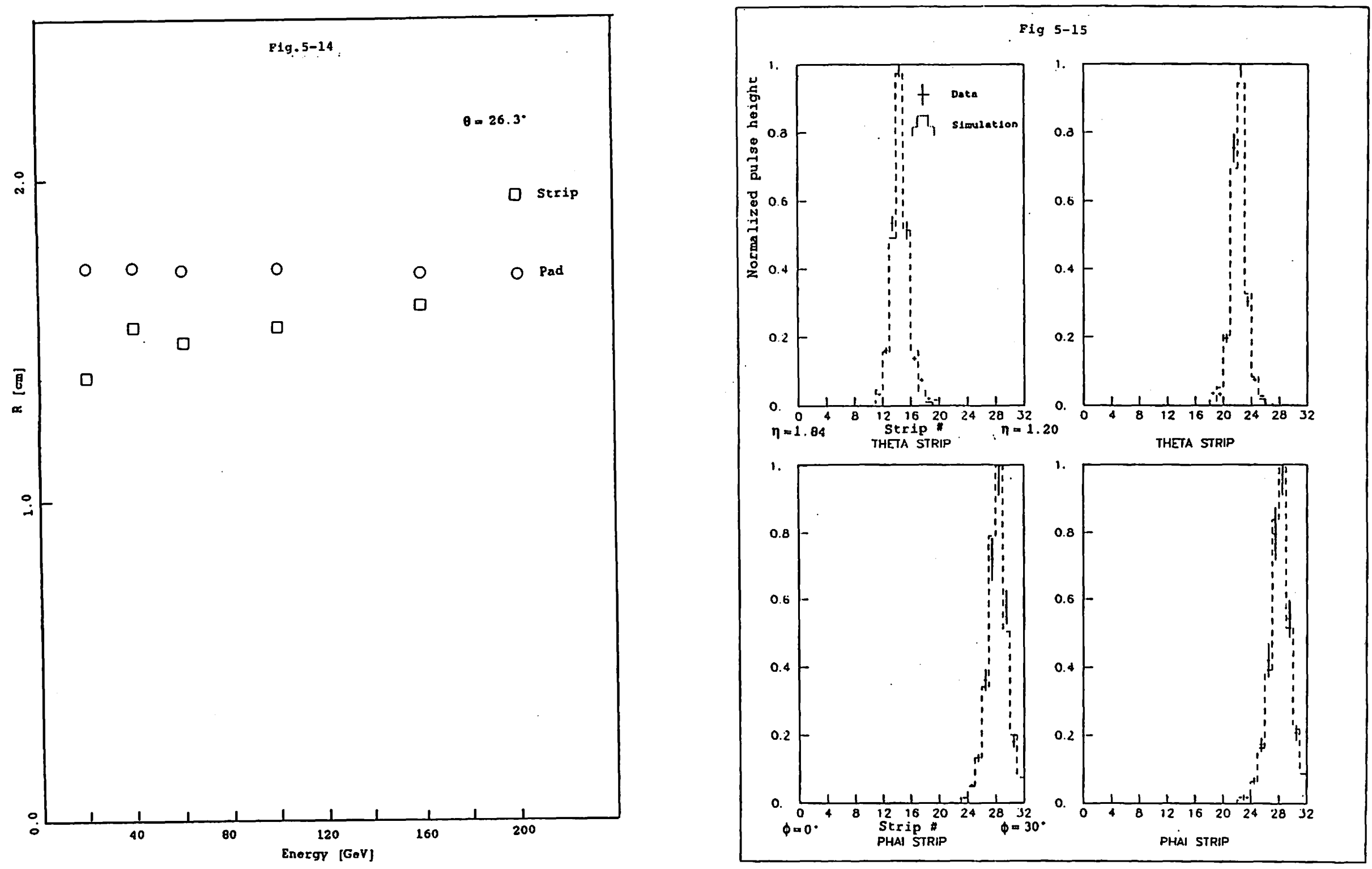

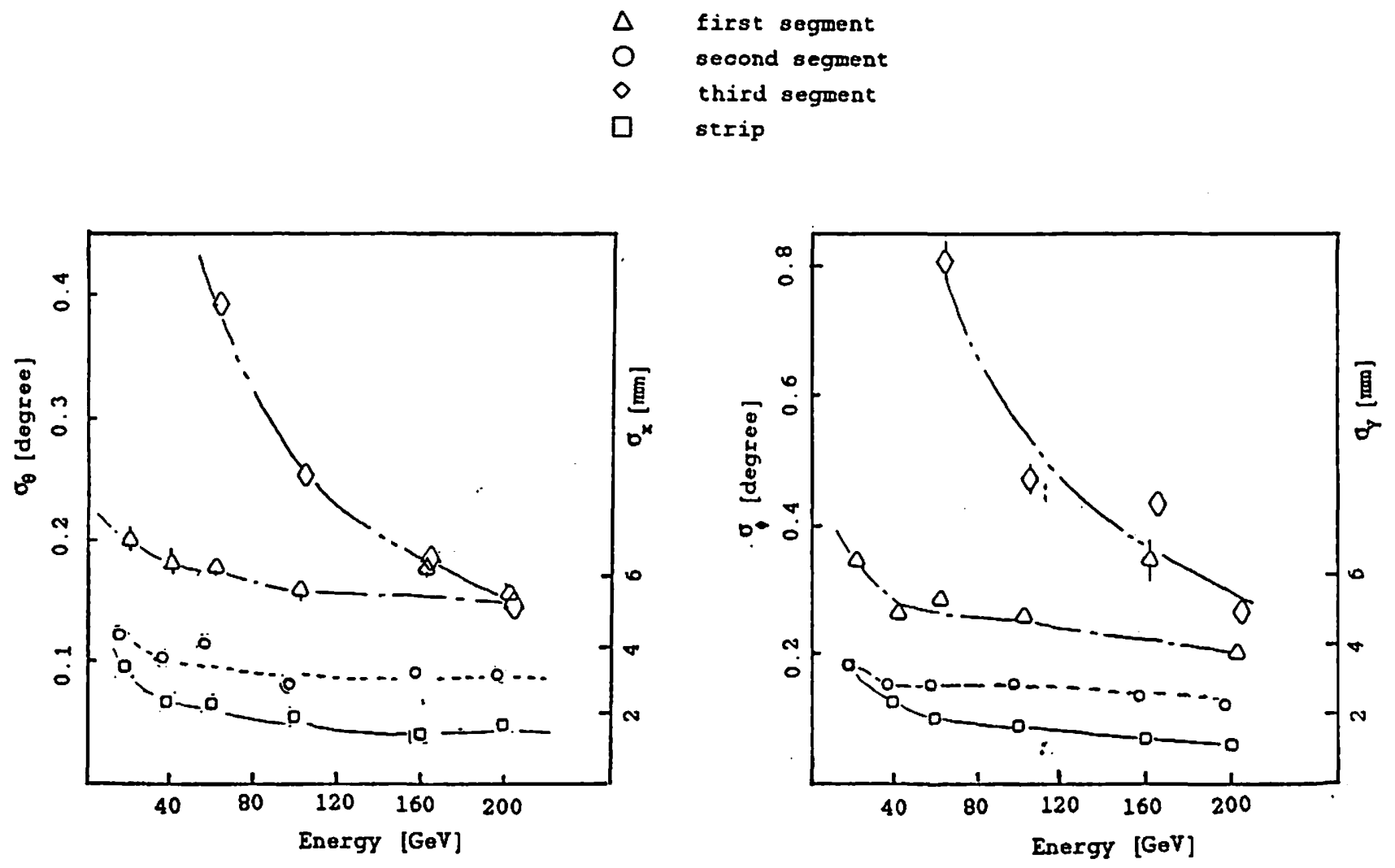

Fig. 5-16

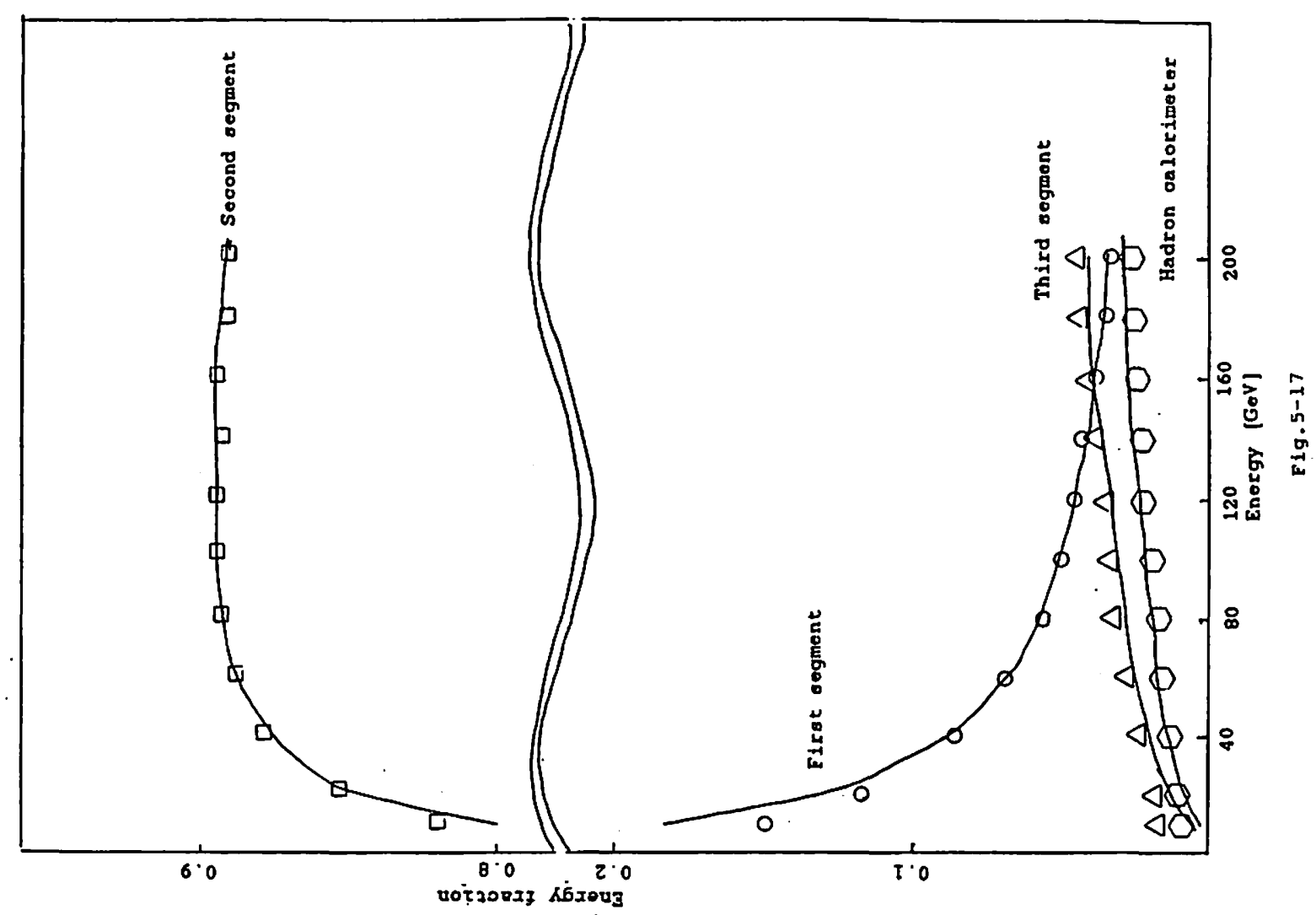




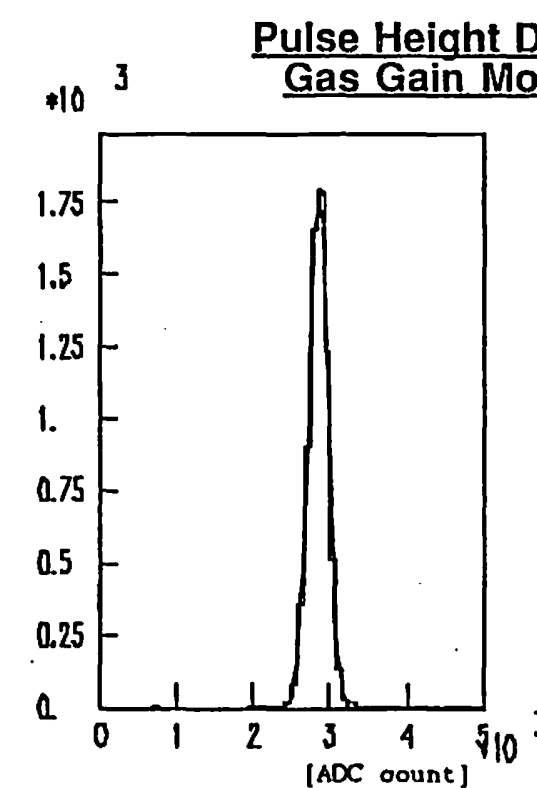

$\frac{\text { Pulse Height Distribution of }}{\text { Gas Gain Monitor Tubes }}$

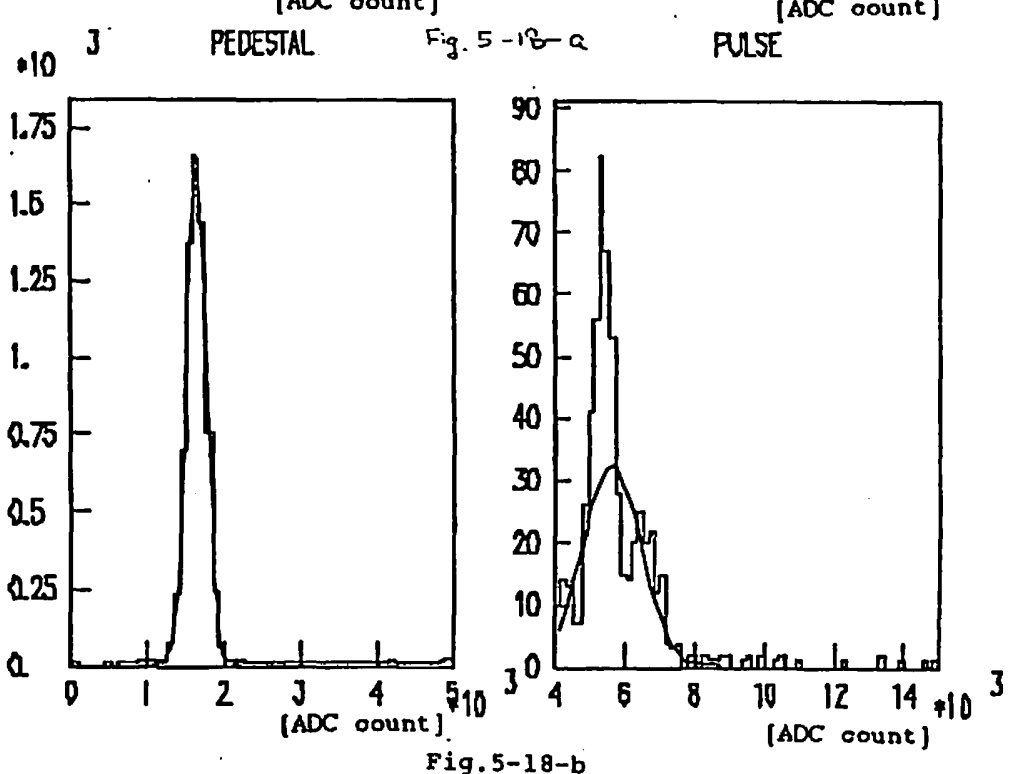

Selection Algorithm of

Gas Gain Monitor Signals
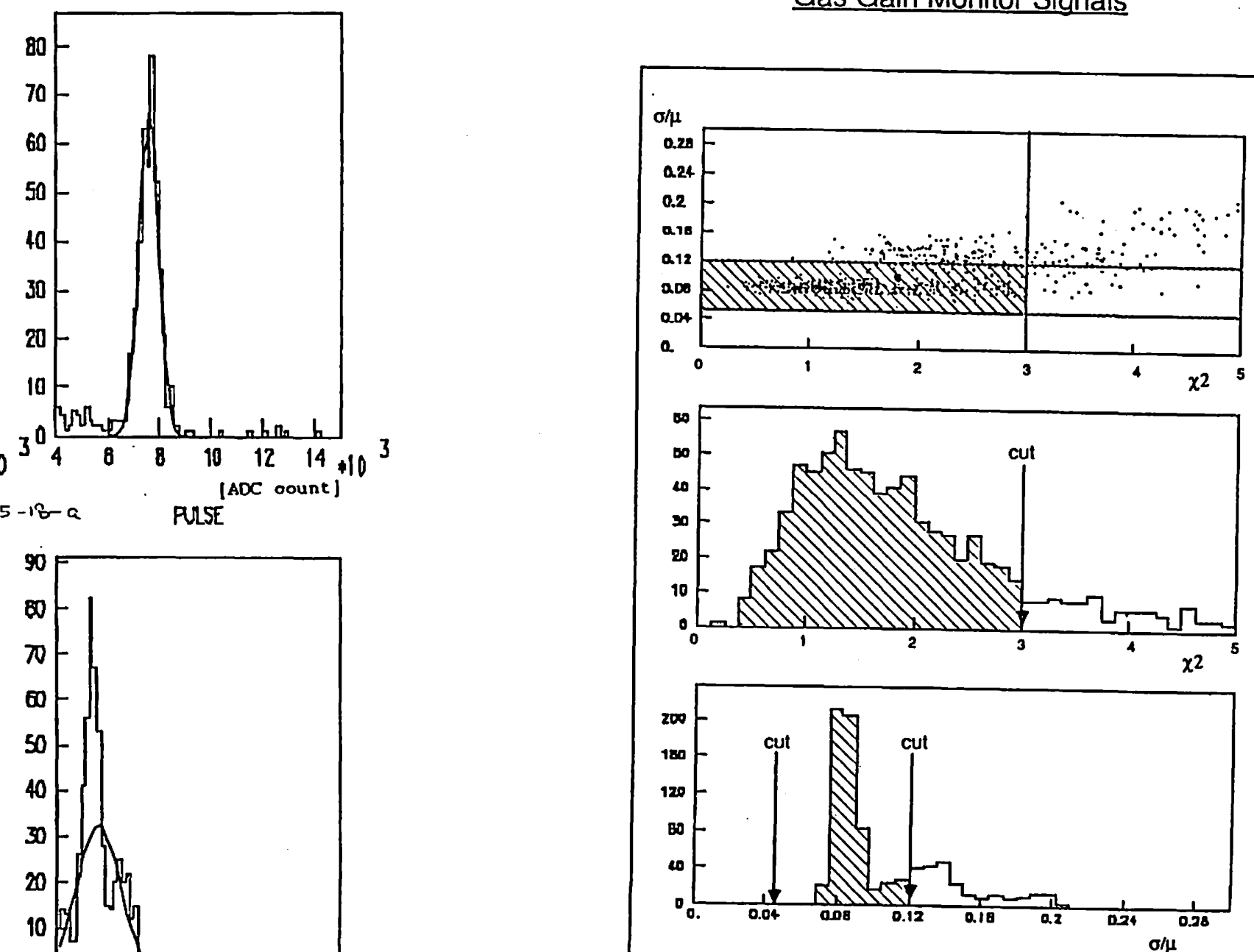

Flg.5-19 
Trace of gas gain

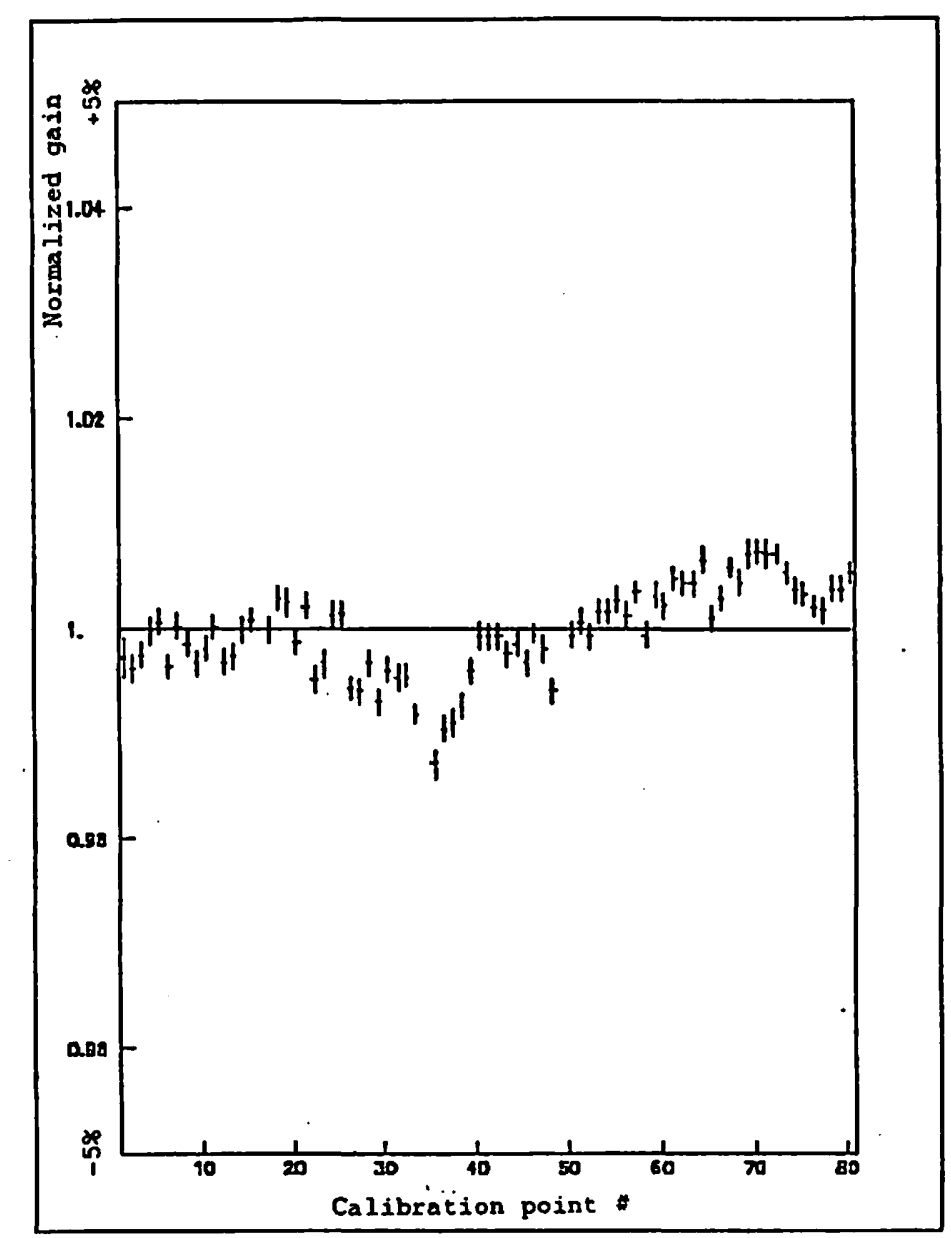

Fig. 5-20

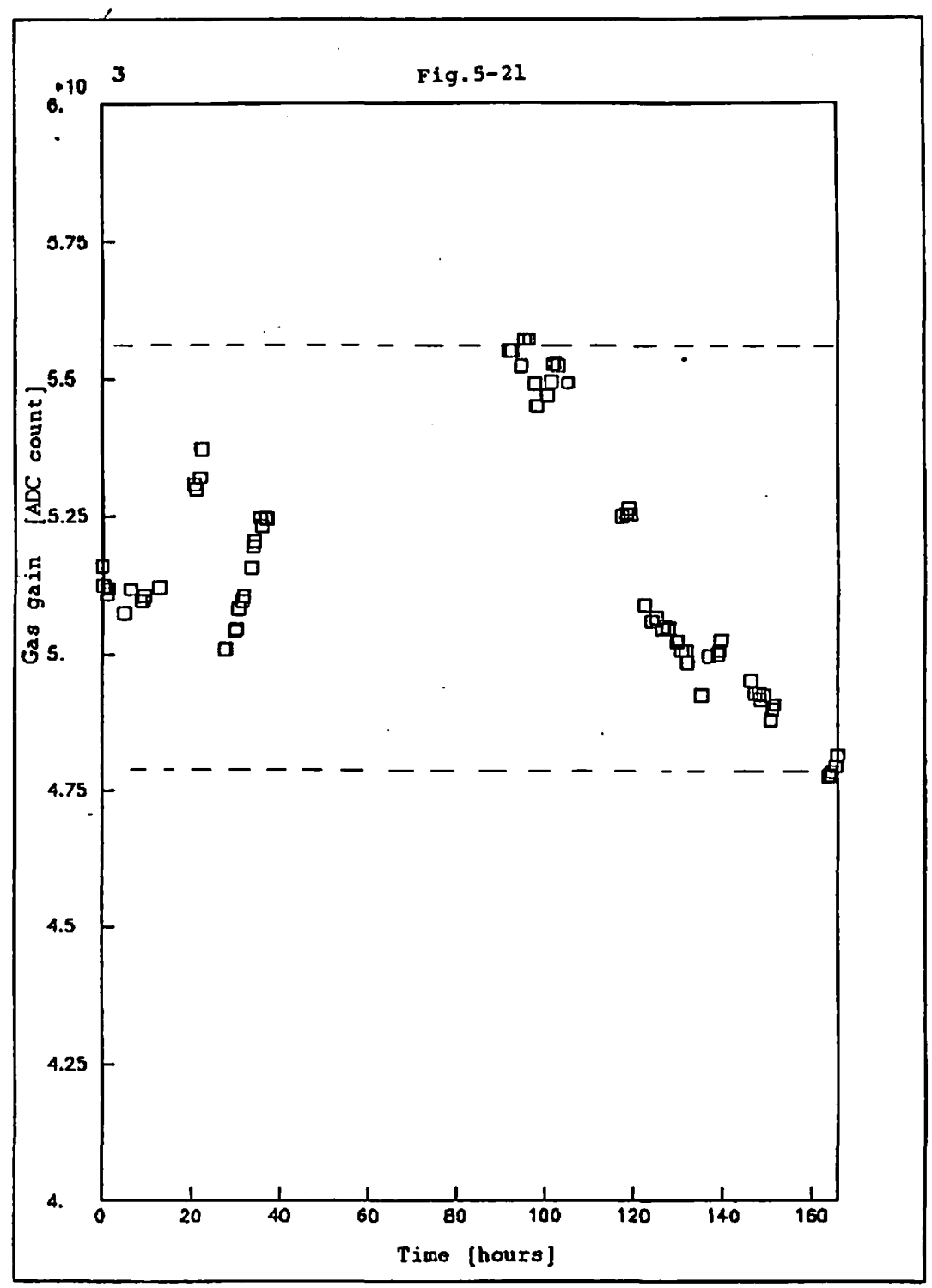




$$
\text { (c) }
$$



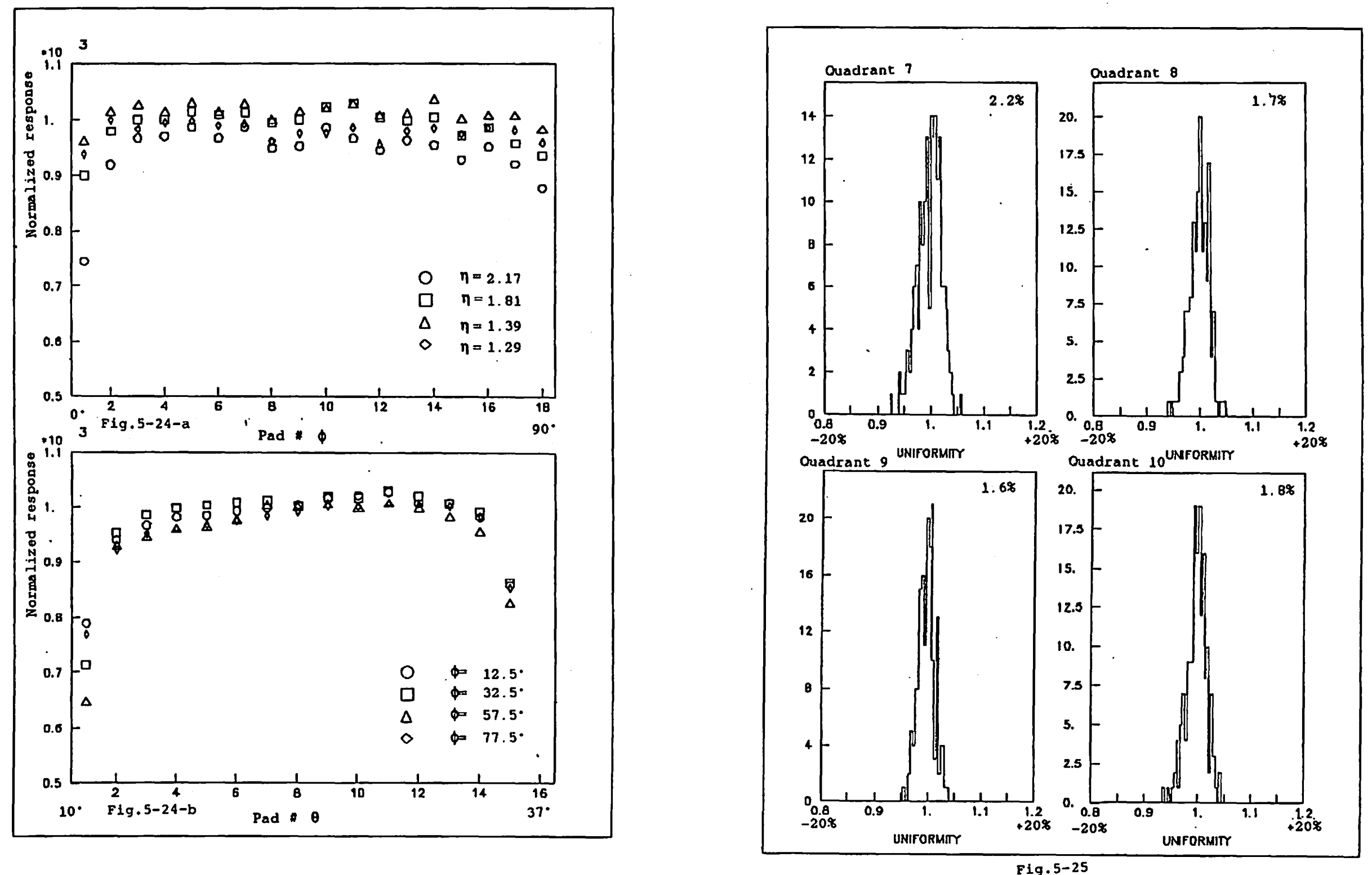

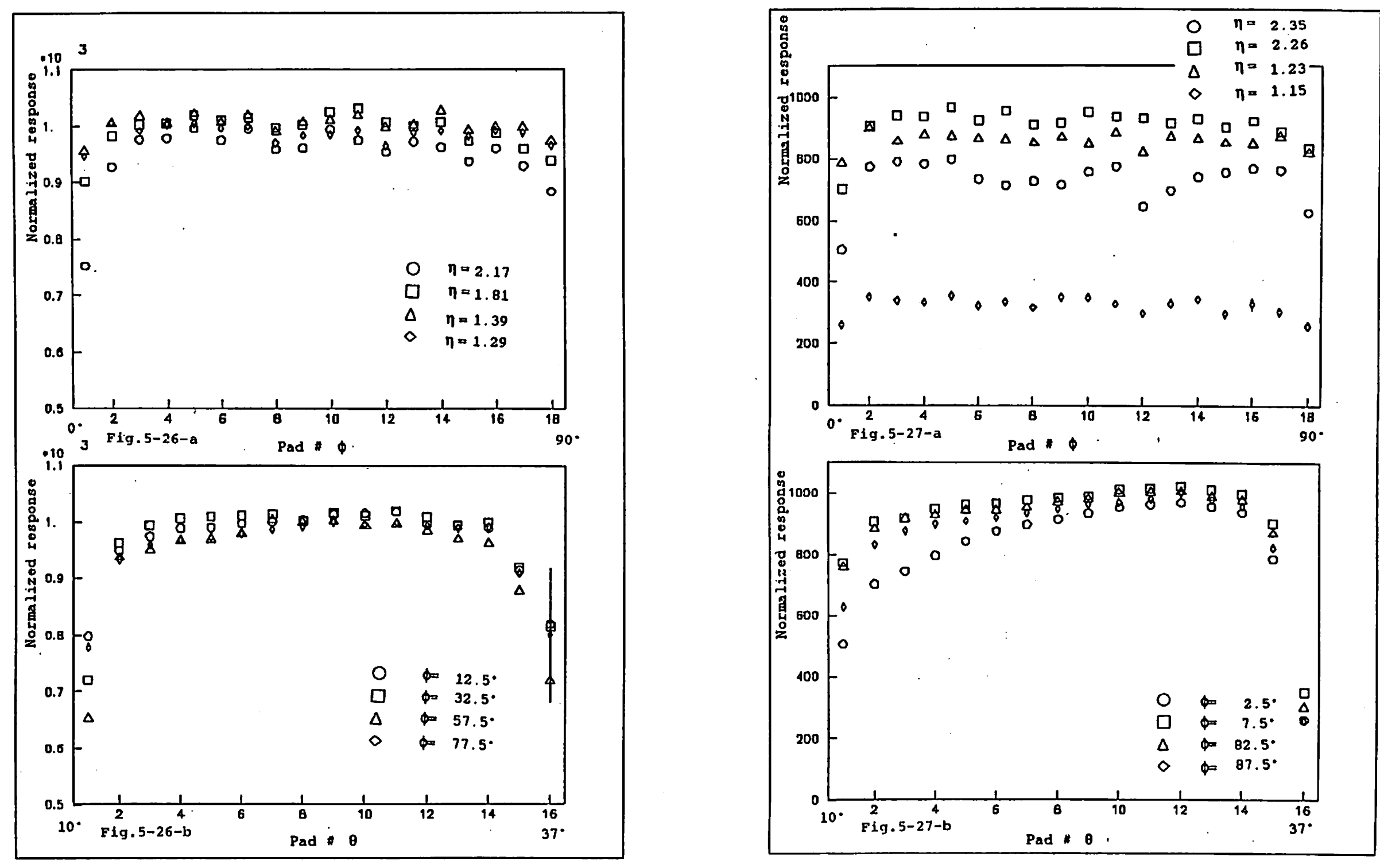
$100 \mathrm{GeV}$ e

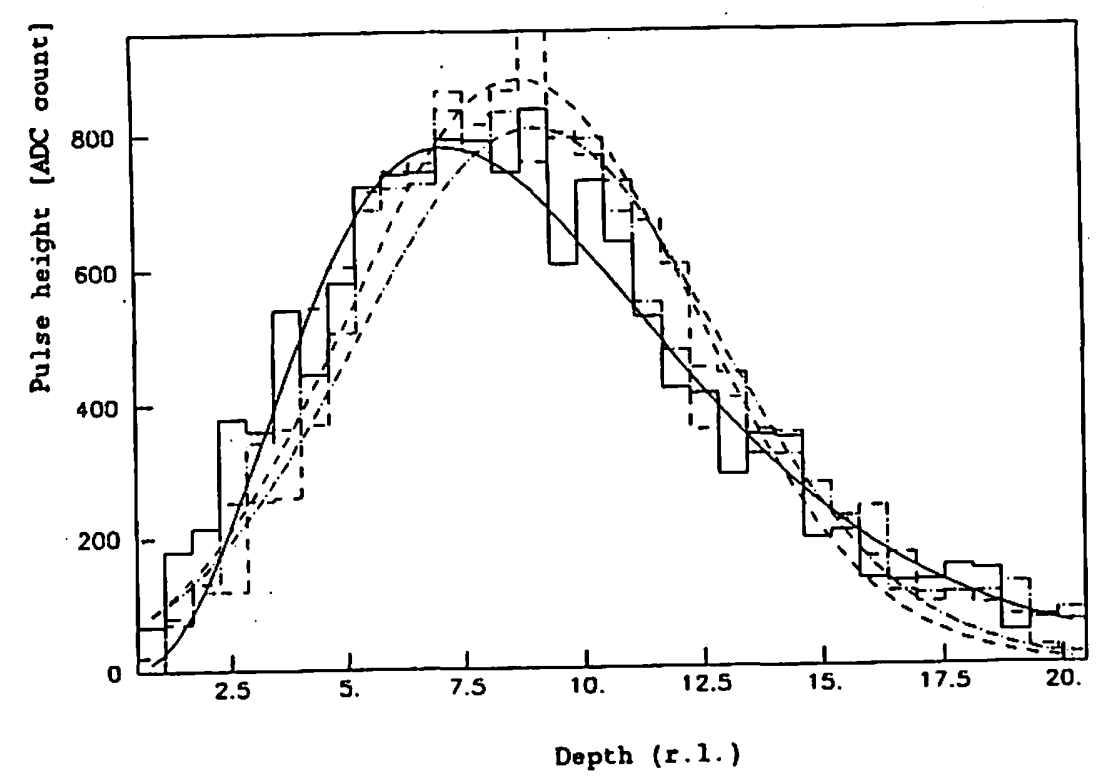

Fig.6-1

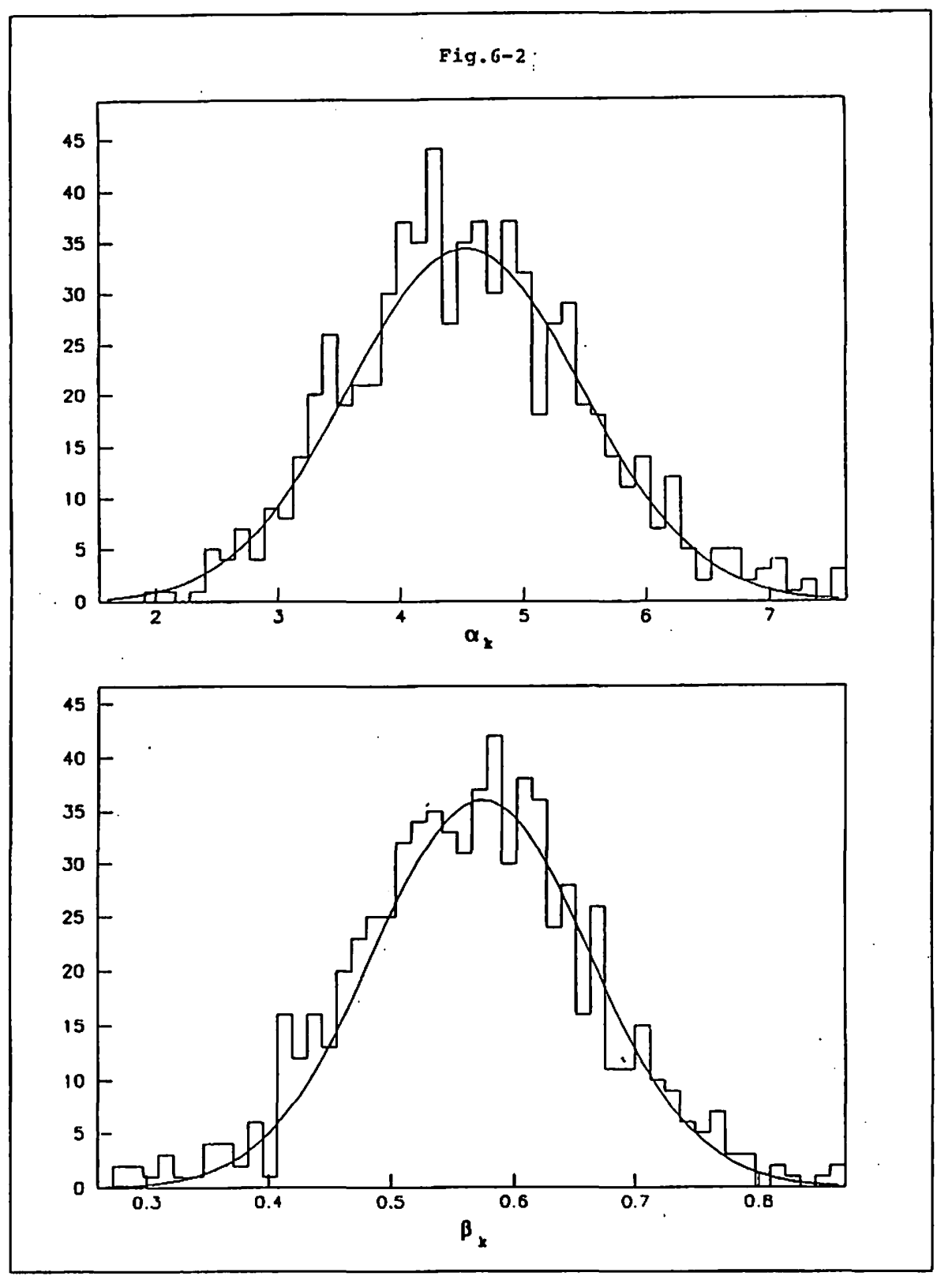


F1g.6-3
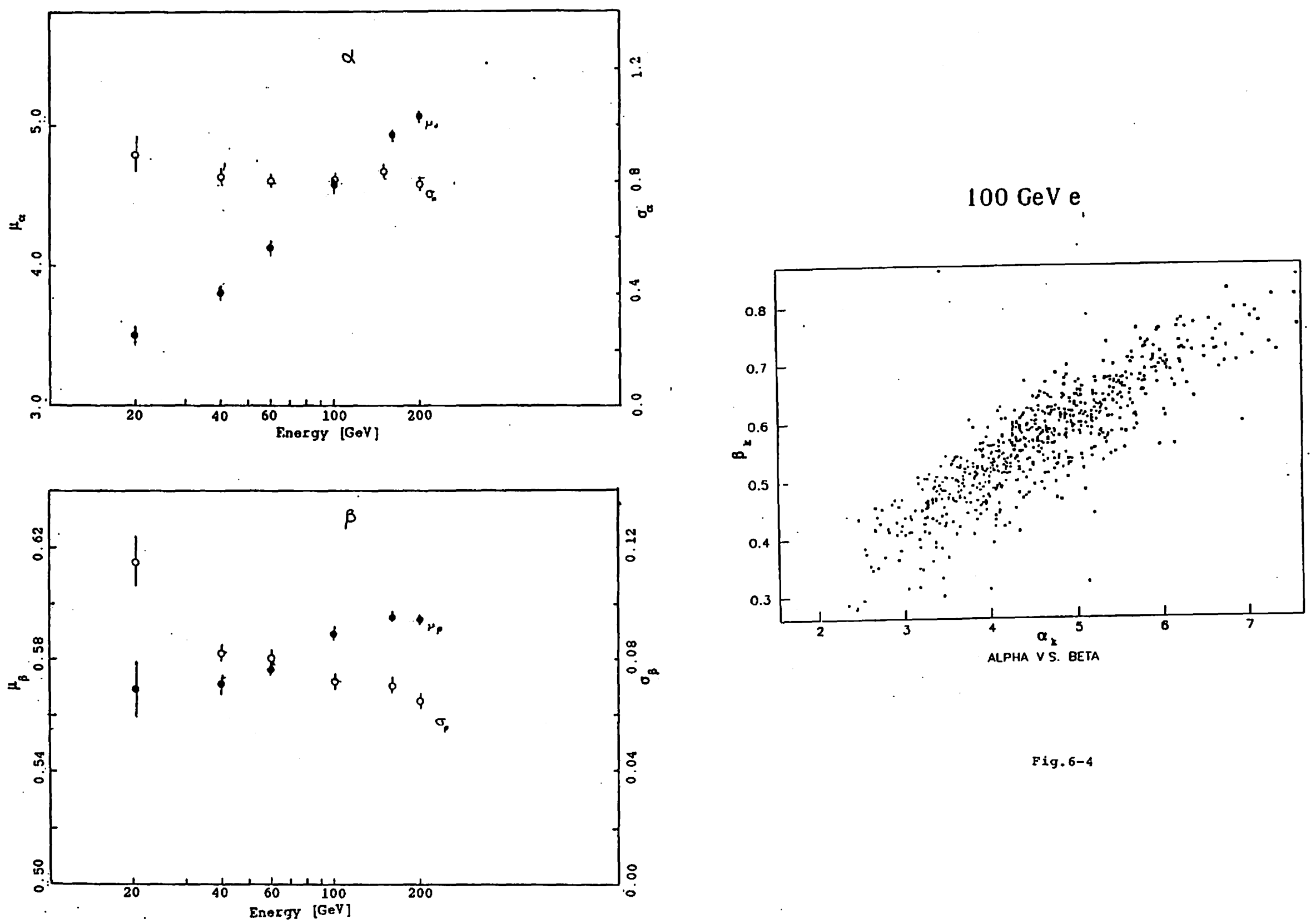

Fig. 6-4 

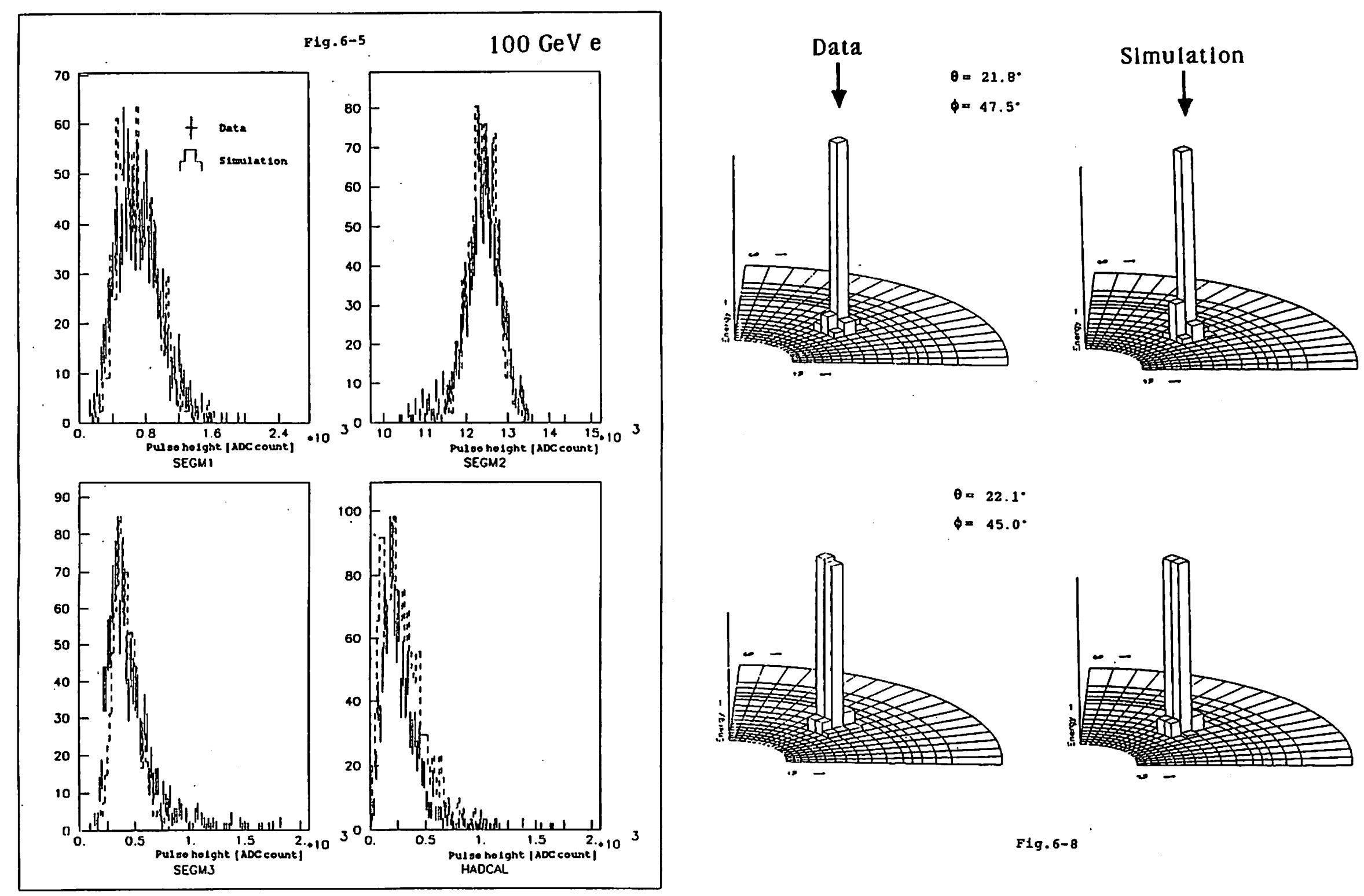

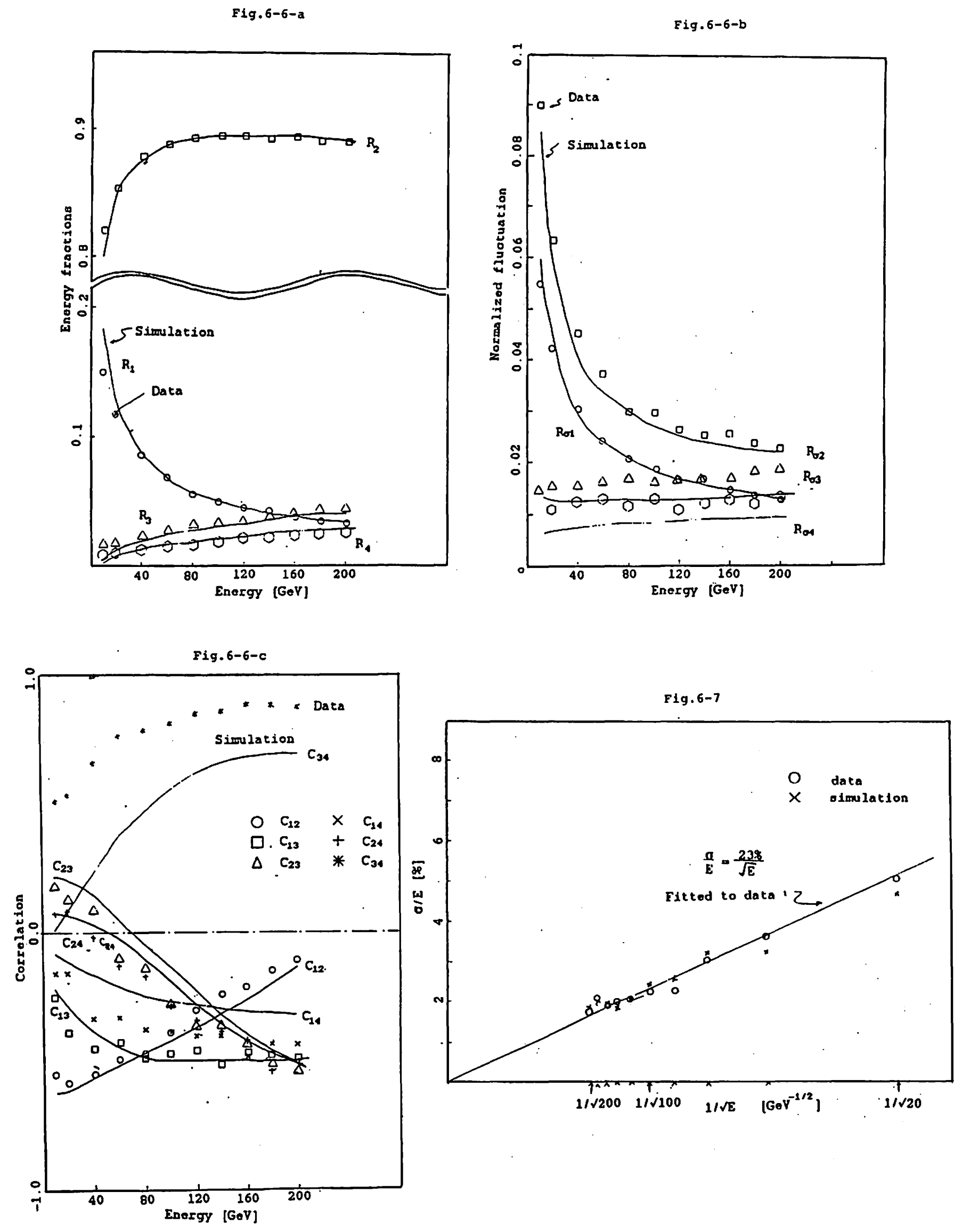

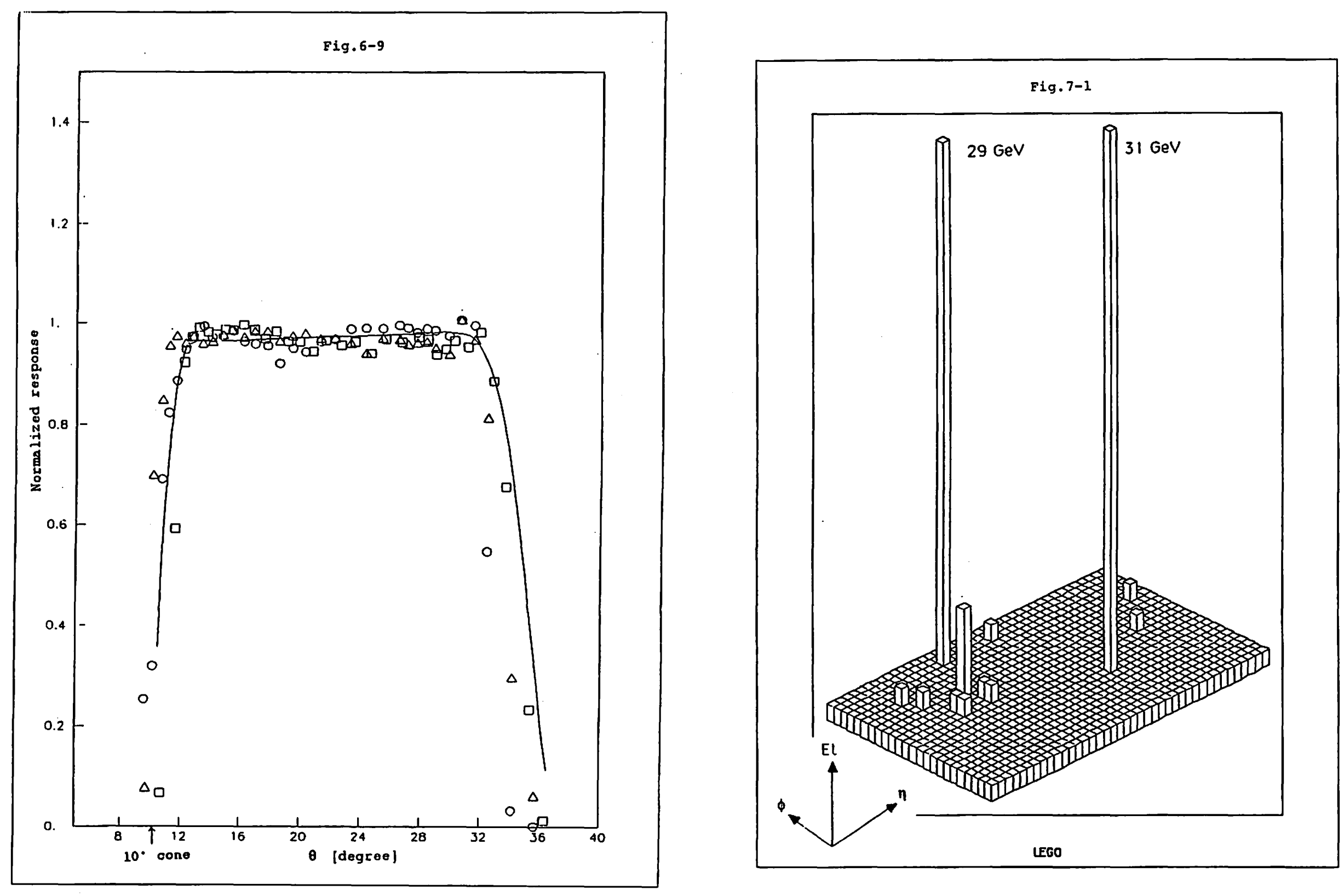

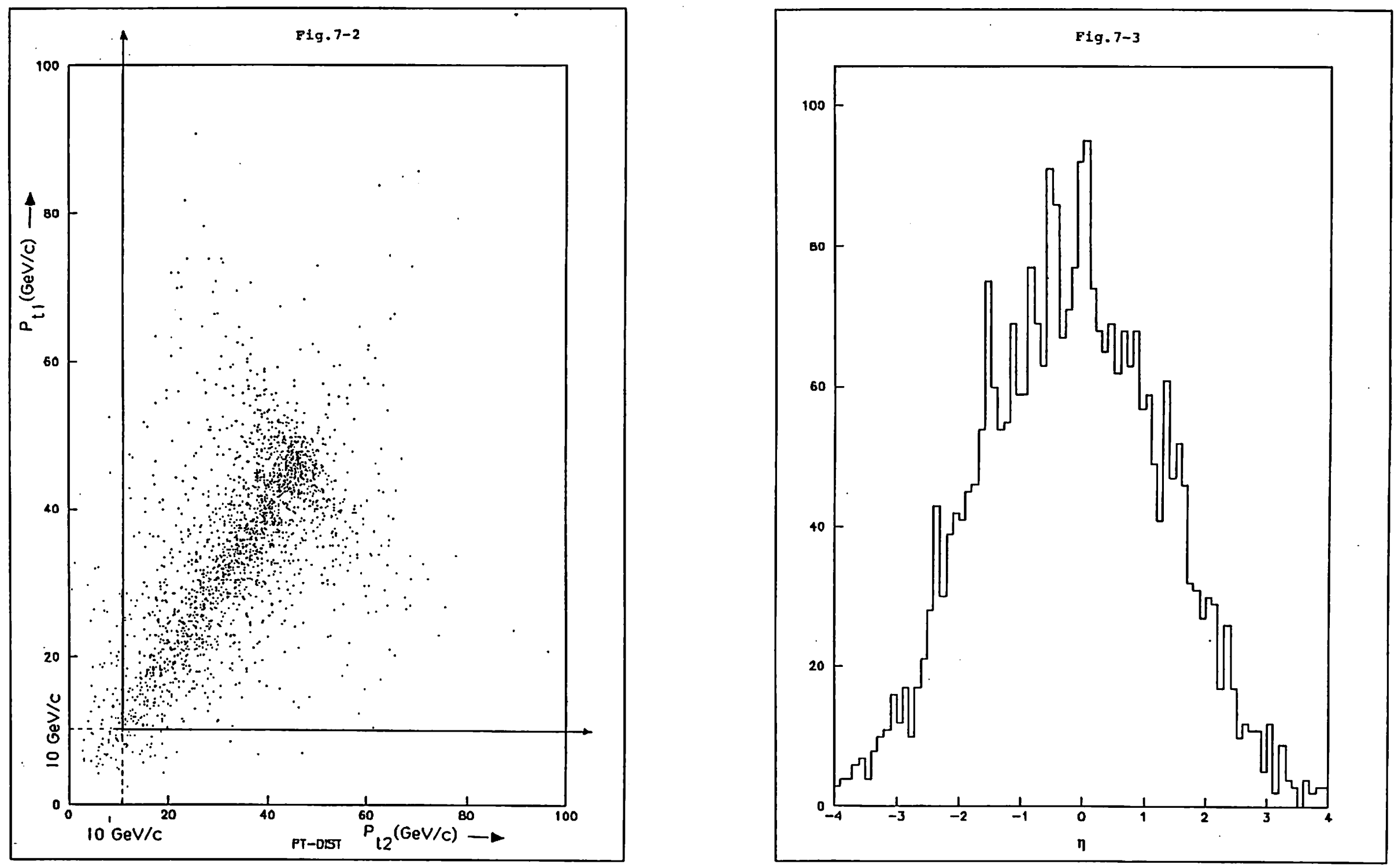

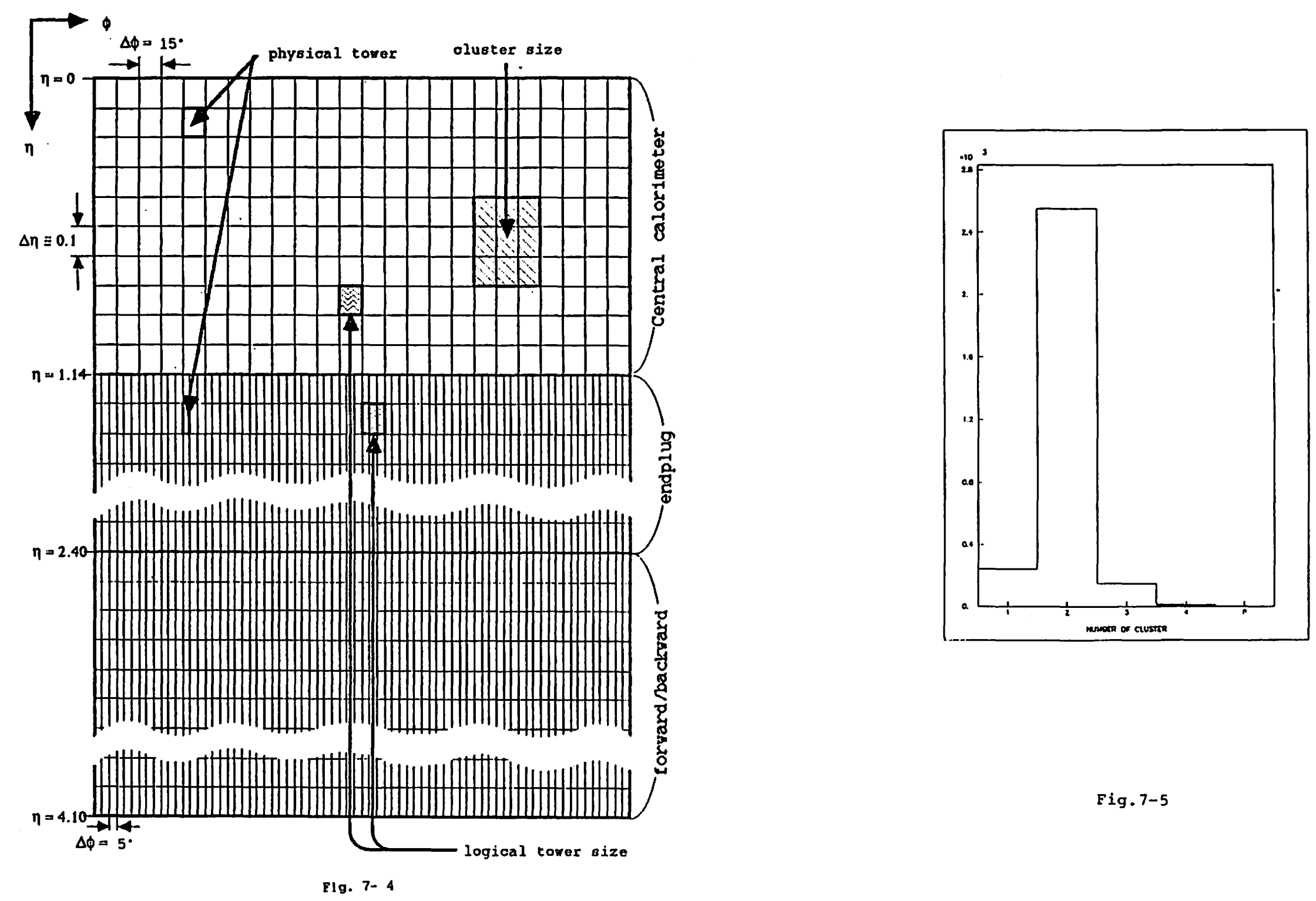

Fig. 7-5 

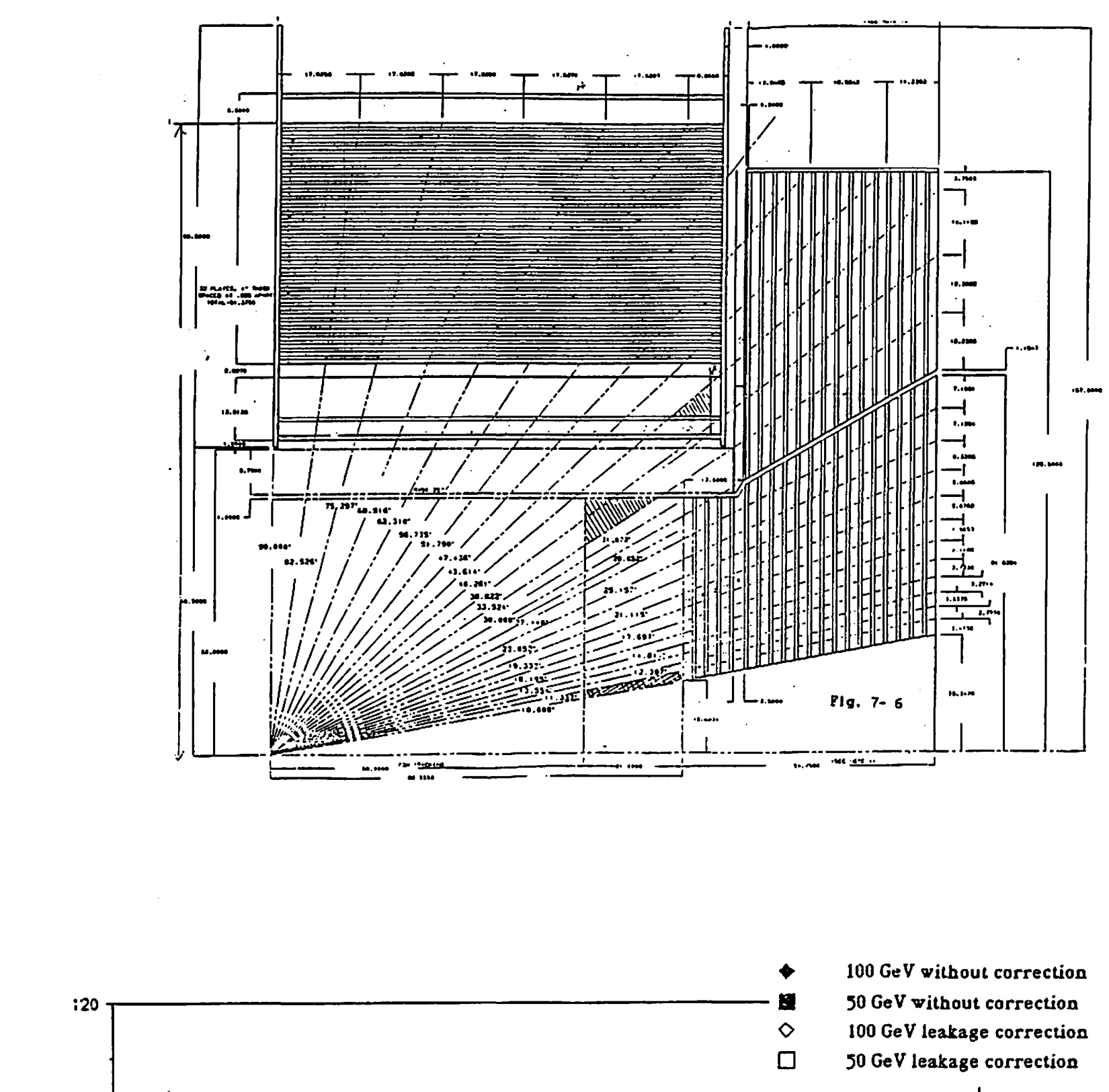
The energy resolution factor of $F / B$ and endplug calorimoter is fixed to 5,15 and $25 \% \cdot \mathrm{VGeV}$.

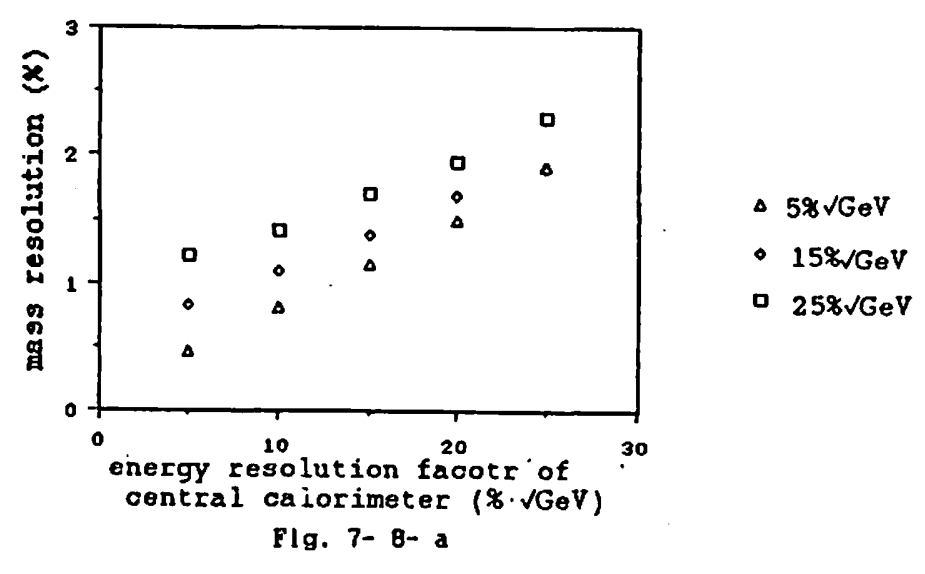

Tne energy resolution factor of central calorimeter is fixed to 5,15 and $25 \% \cdot \sqrt{ } \mathrm{GeV}$.

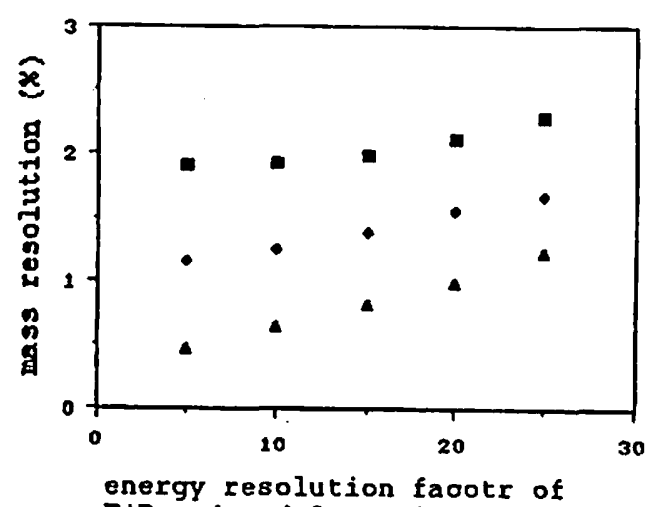

- $58 \sqrt{ } \mathrm{GeV}^{\circ}$

- $15 \pi V G a V$

- 25\% $\sqrt{G e V}$

energy resolution faootr of

$F / B$ and endplug calorimeter $(* \cdot \sqrt{ } \mathrm{GeV})$ Fig. 7- 8- b
The gain uniformity of the F/B calorimeter is fixed to $0 \%, 1 \%, 2 \%$ and $3 \%$.

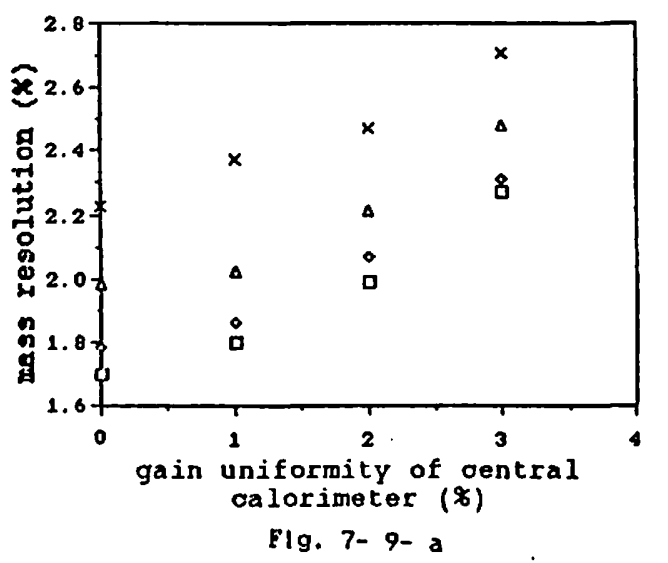

$\times 3 \%$

A $2 \%$

- $1 \%$

- 08

The gain uniformity of the oentral calorimeter is fixed to $0 \%, 1 \%, 2 \%$, and $3 \%$.

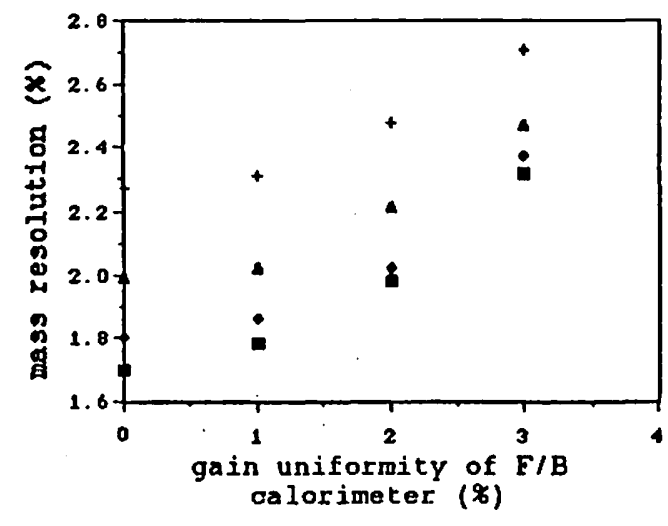

$+38$

- 28

- 18

- 08 


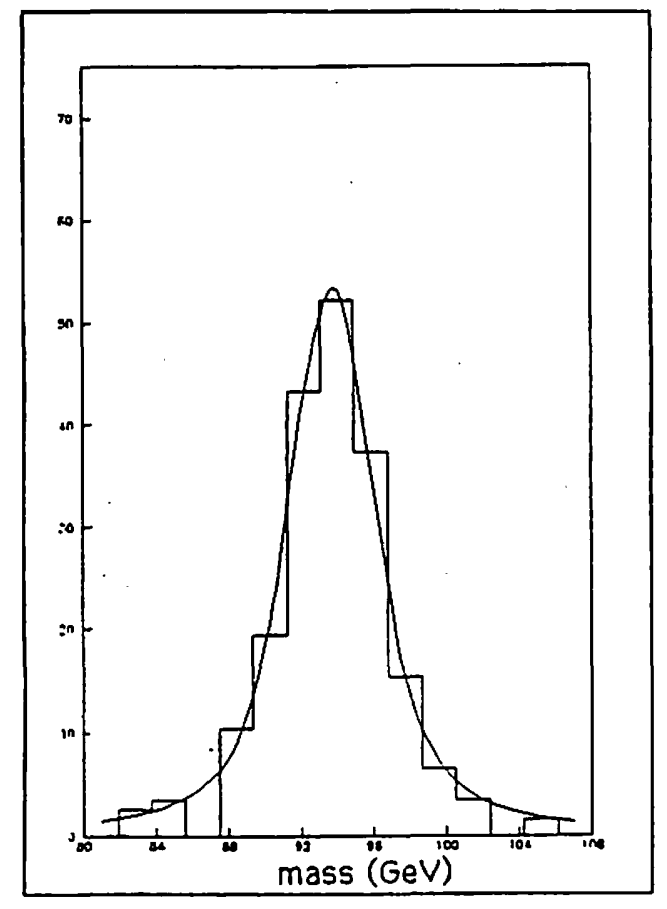

Fig. 7- 10- a

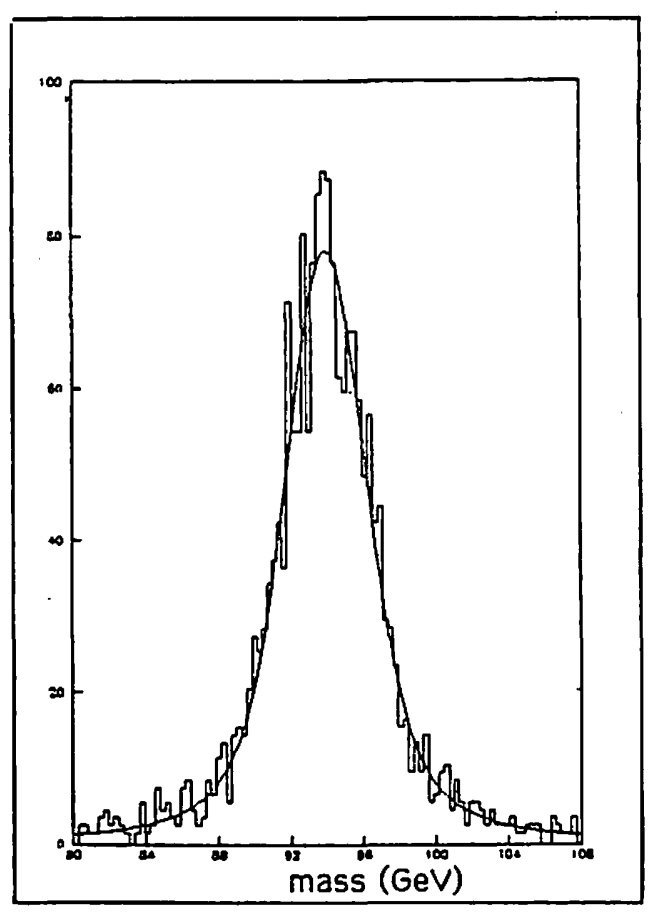

Fig. 7- 10- C

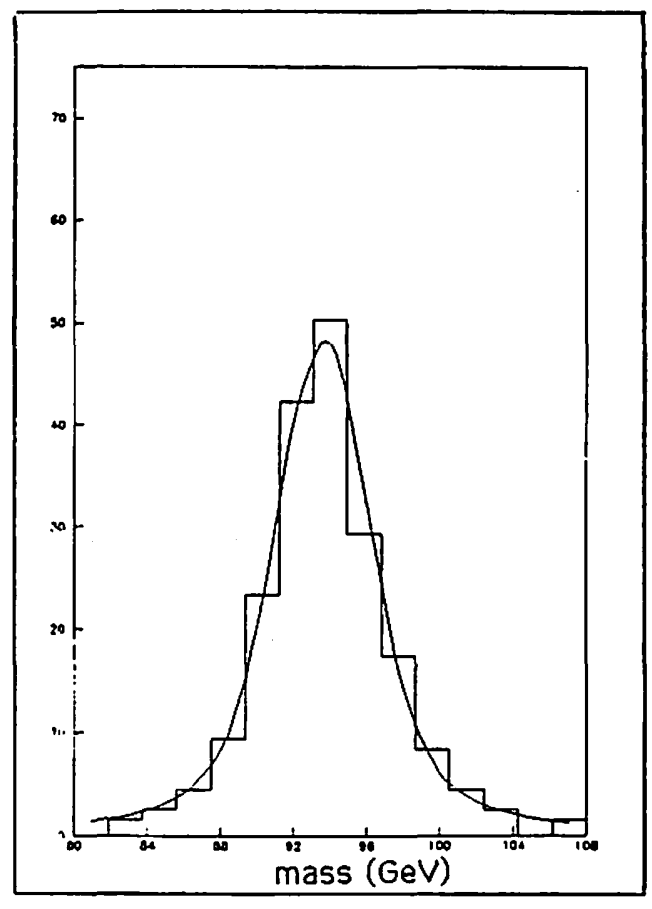

Fig. 7- 10- b

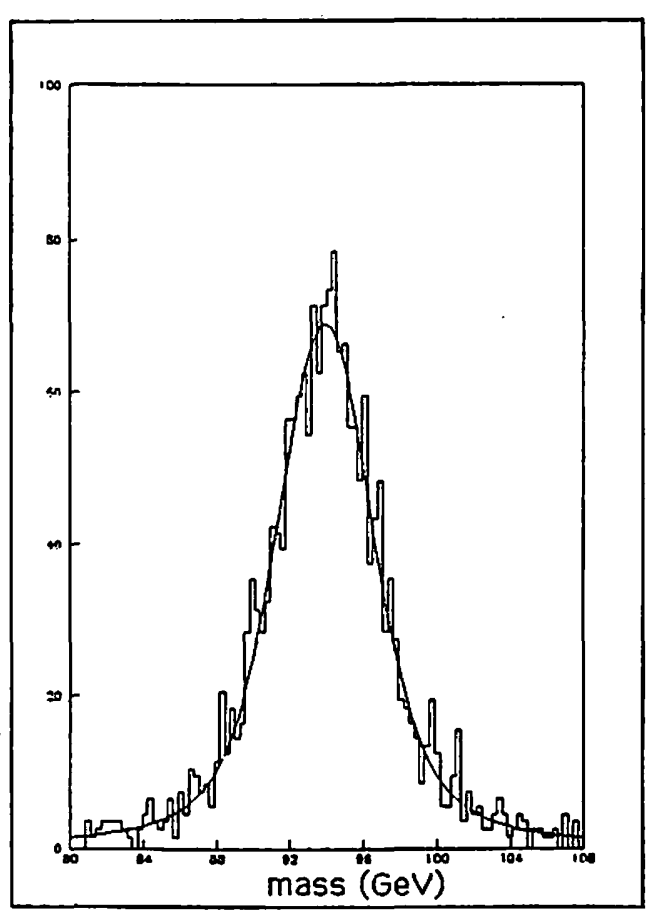

Fig. 7- 10-d 


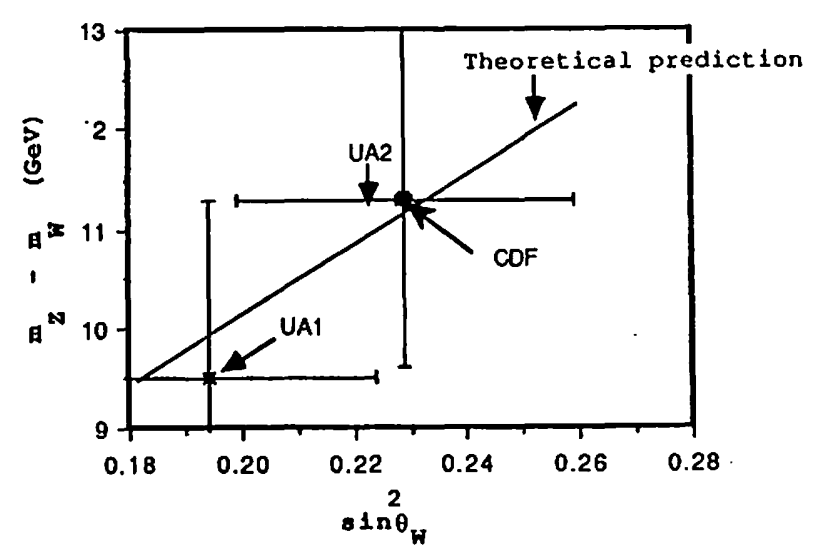

Fig. 7-11

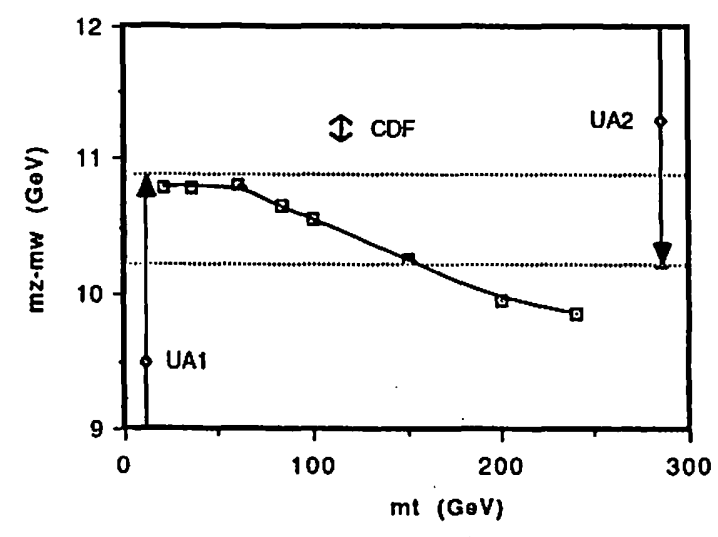

Fig. 7-12

Fig. 7- 11

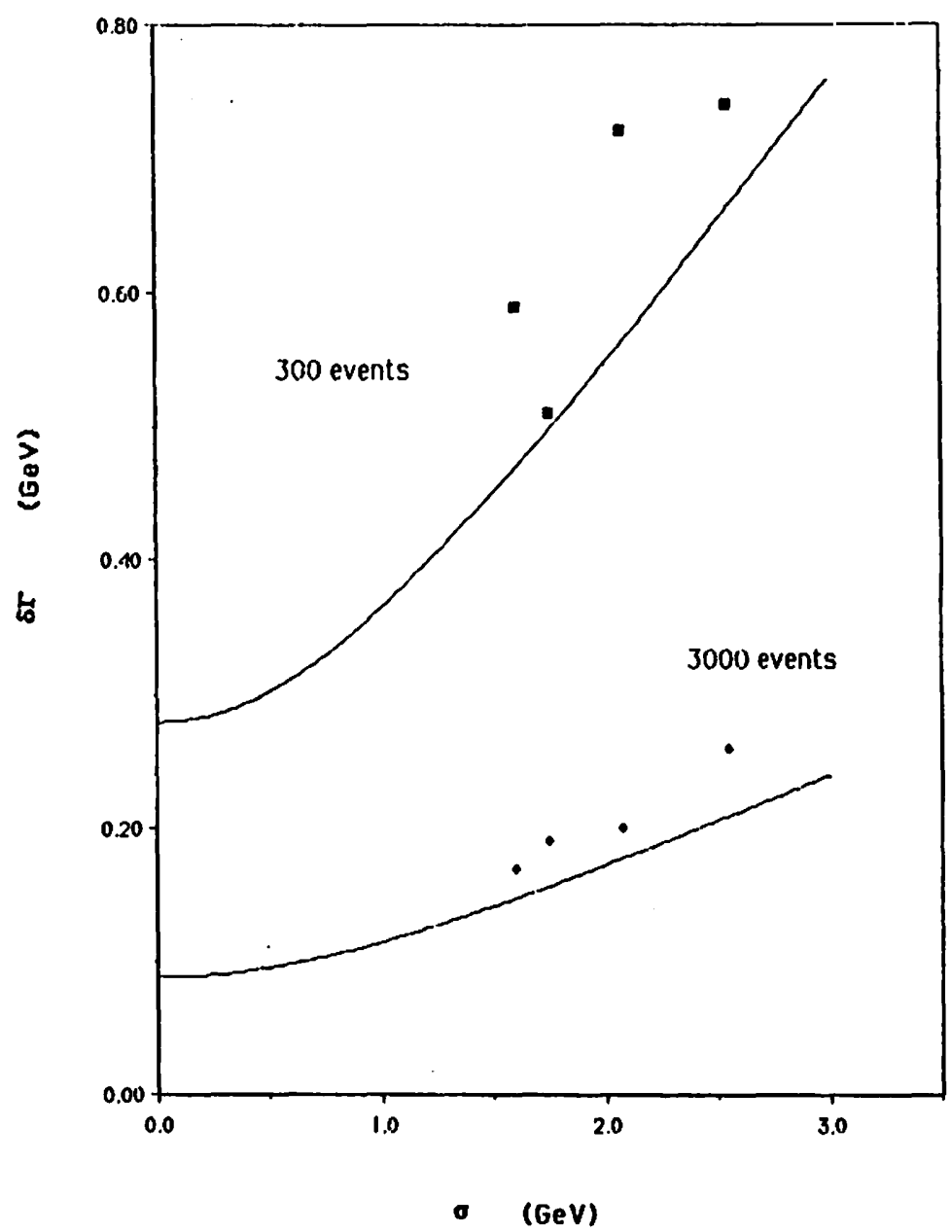


